\section{OPEN ACCESS}

Edited by:

Jose Maria Prieto,

Liverpool John Moores University,

United Kingdom

Reviewed by:

Girish Kumar Gupta,

Sri Sai Group of Institutes

Badhani, India

Vivekananda Mandal,

Guru Ghasidas Vishwavidyalaya, India

*Correspondence:

Jayanta Kumar Patra

jkpatra.officia/@gmail.com

${ }^{\dagger}$ These authors share first authorship

Specialty section:

This article was submitted to

Ethnopharmacology,

a section of the journal

Frontiers in Pharmacology

Received: 12 May 2020 Accepted: 07 September 2020 Published: 08 October 2020

Citation:

Das G, Kim D-Y, Fan C, Gutiérrez-Grijalva EP, Heredia JB, Nissapatorn V, Mitsuwan W,

Pereira ML, Nawaz $M$,

Siyadatpanah A, Norouzi $R$, Sawicka B, Shin H-S and Patra JK (2020) Plants of the Genus Terminalia: An Insight on Its Biological Potentials,

Pre-Clinical and Clinical Studies.

Front. Pharmacol. 11:561248. doi: 10.3389/fphar.2020.561248

\title{
Plants of the Genus Terminalia: An Insight on Its Biological Potentials, Pre-Clinical and Clinical Studies
}

\begin{abstract}
Gitishree Das ${ }^{1 \dagger}$, Do-Yeong Kim ${ }^{1 \dagger}$, Chen Fan ${ }^{2}$, Erick P. Gutiérrez-Grijalva ${ }^{3}$, J. Basilio Heredia ${ }^{4}$, Veeranoot Nissapatorn ${ }^{5}$, Watcharapong Mitsuwan ${ }^{5}$, Maria Lourdes Pereira ${ }^{6}$, Muhammad Nawaz ${ }^{7}$, Abolghasem Siyadatpanah ${ }^{8}$, Roghayeh Norouzi ${ }^{9}$, Barbara Sawicka ${ }^{10}$, Han-Seung Shin ${ }^{11}$ and Jayanta Kumar Patra ${ }^{1 *}$

${ }^{1}$ Research Institute of Biotechnology \& Medical Converged Science, Dongguk University-Seoul, Goyangsi, South Korea, 2 Skin Research Institute of Singapore, Agency for Science, Technology and Research, A*STAR, Singapore, Singapore, ${ }^{3}$ Laboratorio de Alimentos Funcionales y Nutracéuticos, Cátedras CONACYT-Centro de Investigación en Alimentación y Desarrollo, Culiacán, México, ${ }^{4}$ Laboratorio de Alimentos Funcionales y Nutracéuticos, Centro de Investigación en Alimentación y Desarrollo, Culiacán, México, ${ }^{5}$ School of Allied Health Sciences, Research Excellence Center for Innovation and Health Products (RECIHP) and World Union for Herbal Drugs Discovery (WUHeDD), Walailak University, Nakhon Si Thammarat, Thailand, ${ }^{6}$ CICECO-Aveiro Institute of Materials and Department of Medical Sciences, University of Aveiro, Aveiro, Portugal, ${ }^{7}$ Department of Nano-Medicine Research, Institute for Research and Medical Consultations (IRMC), Imam Abdulrahman Bin Faisal University, Dammam, Saudi Arabia, ${ }^{8}$ Ferdows School of Paramedical and Health, Birjand University of Medical Sciences, Birjand, Iran, ${ }^{9}$ Department of Pathobiology, Faculty of Veterinary Medicine, University of Tabriz, Tabriz, Iran, 10 Faculty of Agrobioengineering, Department of Plant Production Technology and Commodities Science, University of Life Sciences in Lublin, Lublin, Poland, ${ }^{11}$ Department of Food Science \& Biotechnology, Dongguk University-Seoul,

Goyangsi, South Korea
\end{abstract}

The evaluation and confirmation of healing properties of several plant species of genus Terminalia based on their traditional uses and the clinical claims are of utmost importance. Genus Terminalia has received more attention to assess and validate the therapeutic potential and clinical approval due to its immense folk medicinal and traditional applications. Various species of Terminalia genus are used in the form of herbal medicine and formulations, in treatment of diseases, including headache, fever, pneumonia, flu, geriatric, cancer, to improve memory, abdominal and back pain, cough and cold, conjunctivitis, diarrhea, heart disorder, leprosy, sexually transmitted diseases, and urinary tract disorders. These are reported to possess numerous biological properties, counting: antibacterial, antifungal, antiinflammatory, antiviral, antiretroviral, antioxidant, and antipa7rasitic. This current research review aims to update the detailed biological activities, pre-clinical and clinical studies of various extracts and secondary metabolites from several plant species under the genus Terminalia, along with information on the traditional uses and chemical composition to develop a promising strategy for their potential applications in the form of medicine or use in modern drug formulations for treating diseases like pneumonia, flu, and other types of viral infections or controlling human contagions.

Keywords: antiviral, biological activities, clinical studies, phytogeography, pneumonia, Terminalia sp. 


\section{INTRODUCTION}

Natural products in medicinal plants are essential sources for drug discovery (Harvey et al., 2015). It has been reported that natural products take up to $35 \%$ of the global medicine market, which is approximately 385 billion US dollars (Calixto, 2019). To discover the medical values of natural products, it is critically important to understand the ethnopharmacological uses of various medicinal plants, as it provides reliable information on the evaluation of natural products existing in those medicinal plants (Buenz et al., 2018). Although the development of modern medicines is quickly growing, there is still a large amount of population preferring herbal medicines than the conventional system of medicines due to their effectiveness, lack of medical alternatives, enhancing cost of modern medicines, and cultural preferences (Heinrich, 2000; Tabuti et al., 2003; Amalraj and Gopi, 2017). Based on the data from WHO, about $80 \%$ of the global population depends on traditional medicine, and $60 \%$ of the Indian population in rural areas use herbal medicines (Amalraj and Gopi, 2017). These natural medicines are generally easy to access, safe, cost-effective, and efficient (Amalraj and Gopi, 2017). Except for the medical values, various plants are also widely used as food (Konczak et al., 2014), health care products (Kim and Song, 2013), veterinary medicine (Upadhyay et al., 2011), possessing extensive impacts on daily life.

Terminalia sp., family Combretaceae, is distributed worldwide, with around 250 species, especially in South Asia, Australia, and South Africa. Among them, more than 50 species are used as food (Fan et al., 2015). A list of some of the important plant species under Terminalia genus with medicinal potential is provided in Supplementary Table 1, and a few of the species are shown in Figure 1. These are some of the most widely used medicinal plants in the global ethnopharmacology such as traditional Chinese, Tibetan, and Indian Ayurvedic medicine system. For instance, fruits of Terminalia ferdinandiana Exell, are rich in (1) vitamin C and thereby being consumed as food in Australia (Konczak et al., 2014). Several Terminalia species exhibit nutraceutical value with numerous health benefits, including the treatment of some diseases (Cock, 2015). For example, fruits of T. bellirica (Gaertn.) Roxb. and T. chebula Retz. usually form Triphala, the well-known polyherbal formulation in Ayurvedic and Thai folk medicine, due to its pharmacological applications as a laxative, detoxifying, and

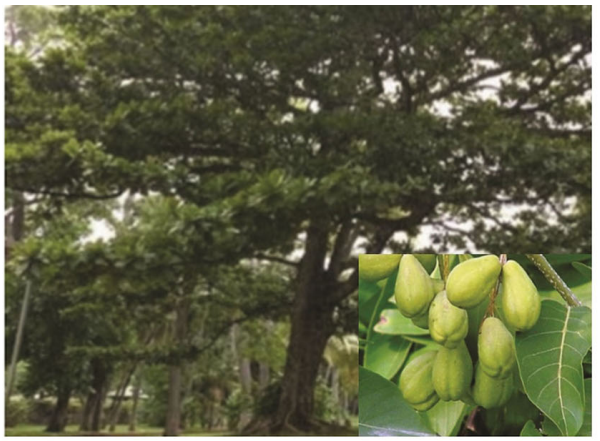

Terminalia chebula Retz. (inset: leaves and fruits)

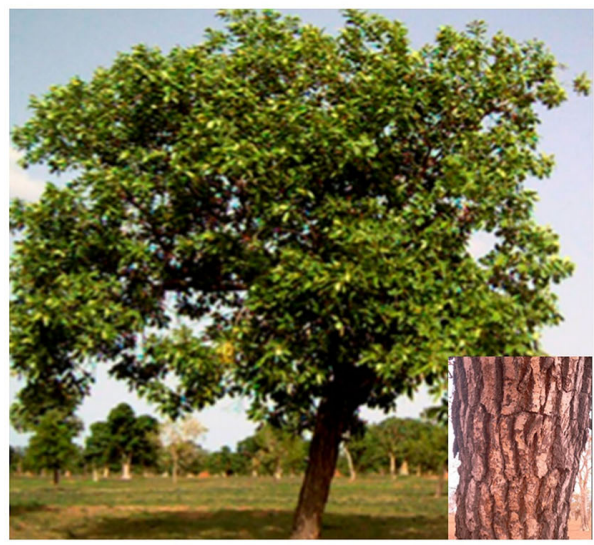

Terminalia brownii Fresen. (inset: stem bark)

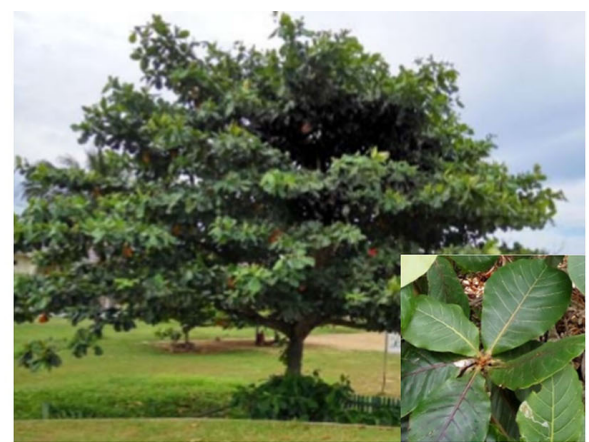

Terminalia catappa L. (inset: leaves)

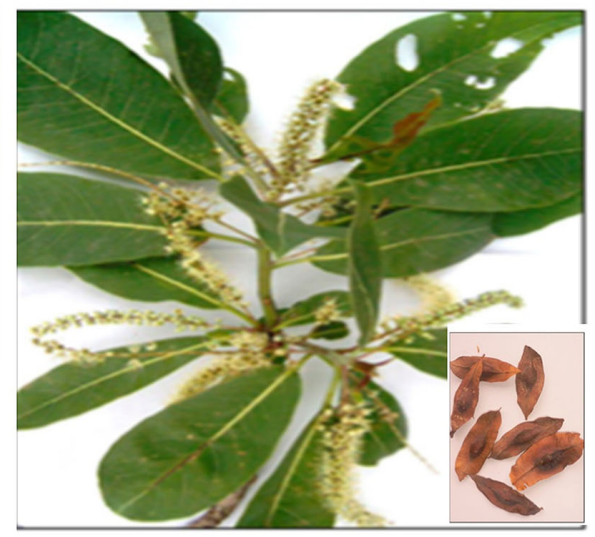

Terminalia brownii Fresen. flowers and leaves (inset: leaves)

FIGURE 1 | Some of the common Terminalia sp. Reproduced under the Creative Commons Attribution License (Afshari et al., 2016; Marjenah and Putri, 2017; Salih et al., 2017). 
rejuvenating effects (Intharuksa et al., 2016). Phytochemical studies in 39 species led to the identification of 368 compounds, including terpenoids, tannins, flavonoids, phenylpropanoids, simple phenolics, among others (Zhang et al., 2019). Some of these compounds demonstrated different bioactivities that were explored through in vitro or in vivo assays. Of note, among these properties, antidiabetic and antiobesity, anticancer, antiinflammatory, antimicrobial, antimalarial, antioxidant, antitumor have been reported in several plant species. It has been documented that T. arjuna (Roxb. ex DC.) Wight \& Arn. is traditionally used for cardioprotective and hepatoprotective purposes in India and Sri Lanka (Kapoor et al., 2014); T. bellirica (Gaertn.) Roxb., is widely used on treating diarrhea (Pandey et al., 2017); Terminalia brownii Fresen., has been used to treat brown-erythematous excoriated papules and plaques (Kibar Ozturk et al., 2018); T. chebula Retz. is widely used to treat dementia, constipation, and diabetes in traditional Indian and Iranian medicine (Jokar et al., 2016), etc. Therefore, the active compounds existing in Terminalia sp. have great potential applications in various diseases. This review presents the muchneeded update on the folk medicinal uses, phytochemistry, chemical composition, and pharmacological applications of numerous plants of genus Terminalia, along with information on the pre-clinical and clinical trials of their compounds. We aim to improve the understanding of the mechanisms underlying the medical use of Terminalia sp., stimulating the use of Terminalia sp. in modern drug discovery.

\section{RESEARCH METHODOLOGY}

To identify information on the biological potential, pre-clinical, and clinical studies of Terminalia sp. this review compiled information from recent literature (2010-2020) from the Scopus, Web of Science, and PubMed databases. The keywords used for the literature research included the terms: Terminalia, antioxidant, cancer, diabetes, antidiabetic, antiobesity, inflammation, antiinflammatory, antimicrobial, antifungal, antiparasitic, nanoparticles, and in vivo studies.

\section{UPDATES ON THE RESEARCH ON TERMINALIA SP. TILL DATE}

It has been known since ancient days that medicinal plants are sources of bioactive compounds. As per the PubMed database, a total of, 201 articles were published on the Terminalia sp., out of which around 191 articles were published during the year 20102020 (Figure 2). Among these articles, maximum was available as full texts whereas only three articles were reviews and one article is on the clinical trial.

Based on the objectives of the current review, the present article focuses on Terminalia sp., belonging to the family Combretaceae, possesses various bioactive properties including antibacterial, antifungal, antiparasitic, antidiabetic, anticancer, antioxidant activity along with several potential chemical compounds that could be of significant importance in the clinical sector. As per the literature search on various databases such as PubMed, most of the previously published articles are reported on the biological activity of Terminalia sp. During the year 2010 to 2020, and this is presented in Figure 3 (https:// pubmed.ncbi.nlm.nih.gov/?term $=$ Terminalia + sp.\&filter $=$ ds1.y_ 10, 15 July 2020).

It has been proved that the phytochemicals presented in the plant species have been the most popular research nitch investigated thus far (Figure 3). The plant species is worldwide distributed, with

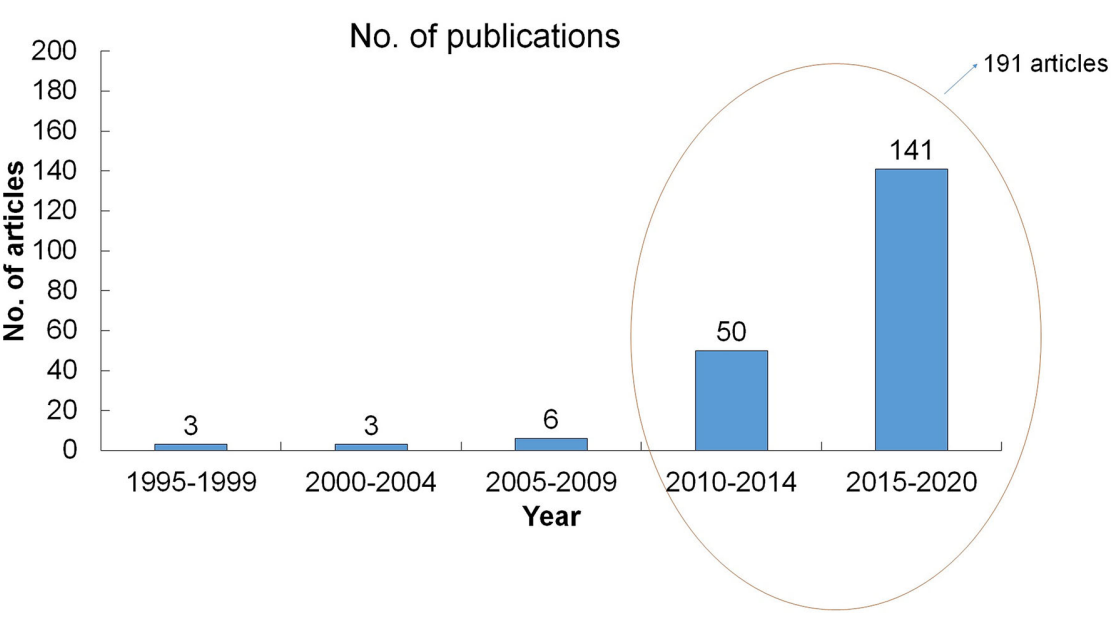

FIGURE 2 | A detailed list of publications on Terminalia sp. Till date. The information was collected from PubMed (https://pubmed.ncbi.nlm.nih.gov/?term=Terminalia +sp.\&filter=ds1.y_10, 15 July 2020). 


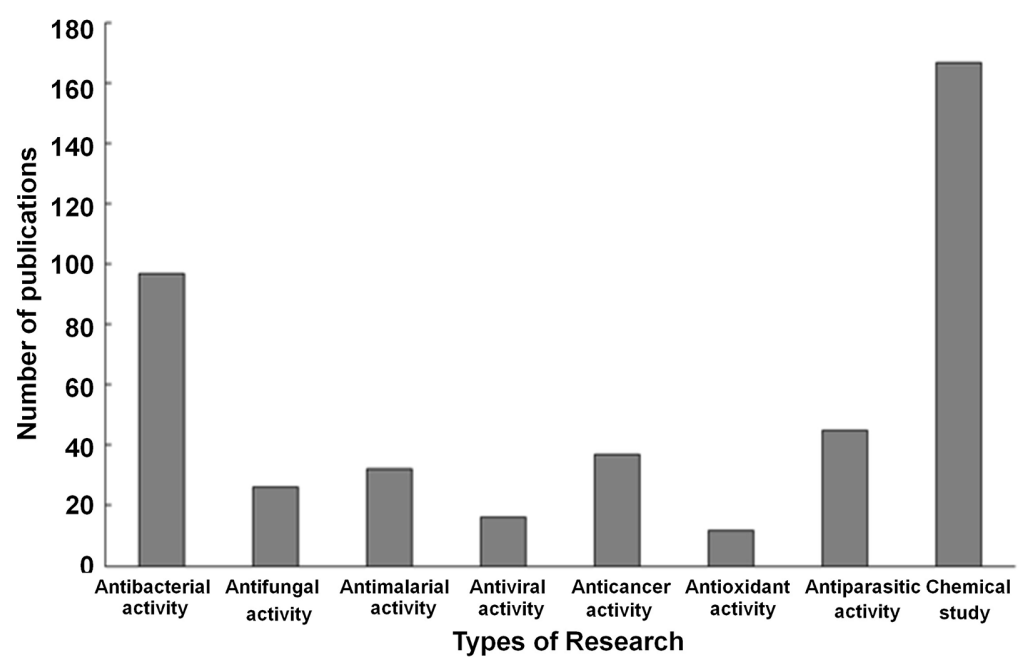

FIGURE 3 | Trends in Terminalia sp. research within 2010-2020. The information was collected from PubMed (https://pubmed.ncbi.nlm.nih.gov/?term=Terminalia +sp.\&filter=ds1.y_10, 15 July 2020).

more than 250 species in Asia, Himalayas, Madagascar, Australia, and Southern Africa. Importantly, the ethnopharmacological use of the plant species is well-known in India named Ayurvedic medicine as well as in Chinese medicine. Also, some of the Terminalia sp. fruits including Terminalia bellirica (Gaertn.) Roxb. and Terminalia chebula Retz. have been used as a polyherbal formulation in Ayurvedic and Thai folk medicine named Triphala (Intharuksa et al., 2016). Therefore, Terminalia sp. phytochemicals have been studied by many researchers. Several phytochemical groups including glycosides, flavonoids, tannins, phenols, saponin, carbohydrates, and proteins have been found in the plant species (Abraham et al., 2014). Furthermore, pure new compounds and pure well-known compounds have also been isolated from several Terminalia sp. (Wright et al., 2016). According to the varieties of chemical constituent compounds present in the Terminalia plant species, a study on the phytochemicals of the Terminalia sp. including the isolation of a new compound to be used as a novel drug for the treatment of diseases is an important area of research. The antibacterial activity of Terminalia sp. is the second popular research determined by the researchers (Figure 3). Terminalia sp. has been used in traditional medicine to treat bacterial infectious diseases including diarrhea, dysentery, pneumonia, and sore throats (Eloff et al., 2008). Therefore, the trend of the bioactivity study on Terminalia sp. is an important area of research. It has been reported that the growth of airborne pathogens including MDR Acinetobacter sp. and MDR Pseudomonas aeruginosa is suppressed when the microorganisms were treated with Terminalia bellirica (Gaertn.) Roxb. (Dharmaratne et al., 2018) and Terminalia chebula Retz. (Sharma et al., 2012) fruit extracts.

Malaria, the number one killer parasitic disease, is the world's most significant protozoan disease. It has been reported by the World Health Organization that 228 million cases of malaria worldwide occurred in 2018. In addition, Haemonchus contortus and Trypanosoma brucei are some of the major causes of human morbidity and mortality in Africa and Some part of Asia. It has been noticed that Terminalia sp. are one of the most important plant ingredient in traditional medicine to treat several infectious diseases such as malaria (Malterud, 2017). Of this, antiparasitic particularly antimalarial activities of Terminalia sp. have been studied across the world. Also, the anticancer activity of Terminalia sp. has been focused mainly since cancer is one of the most important human diseases that causes public health concerns worldwide. The diseases are abnormal cell growth with the potential to spread to other organs of the human body. Importantly, cancer is considered one of the major noncommunicable diseases leading to high morbidity and mortality rates as well as a huge impact of economical loss on a large scale. In line with this report, the anticancer activity of Terminalia sp. has then been a hotspot in search of novel anticancer therapy. Basing on the above insight into the importance of the Terminalia sp., it is evident to compile a detailed report on the medicinal potential of the various species of Terminalia sp. and its important phytoconstituents and pharmacological importance.

\section{TERMINALIA SP. MEDICINAL POTENTIAL (FOLK MEDICINAL USES, TRADITIONAL USES)}

The genus Terminalia sp. are widely used in various traditional medicines such as traditional Chinese medicine, Tibetan medicine, and Indian Ayurvedic medicine practices (Zhang et al., 2019). Terminalia sp. is found to possess various bioactivities such as antitumor, antiinflammatory, anti-bacterial, antifungal, and 
antiviral properties (Zhang et al., 2019). There are several species of plants belonging to the genus Terminalia, and some of these plant species and their traditional uses are discussed below.

Terminalia argentea Mart. is an aboriginal tree growing in various regions of Brazil. Leaf of T. argentea Mart. is traditionally used to treat digestion and respiratory-related diseases in Brazil. It has also been reported that the hydroethanolic extract from the leaves of T. argentea Mart. has no cytotoxicity in CHO-K1 and AGS cells in vitro (Beserra et al., 2018). T. arjuna (Roxb. ex DC.) Wight \& Arn. is propagated by seeds and grows almost in all types of soils, however, humid, fertile loam and red lateritic soils are preferred (Dwivedi and Chopra, 2014). T. arjuna (Roxb. ex DC.) Wight \& Arn. is an endemic tree widely found in India and Sri Lanka and used traditionally for cardioprotective and hepatoprotective purposes. In the indigenous medicine system, T. arjuna (Roxb. ex DC.) Wight \& Arn. is widely documented for its use in treating cardiovascular diseases (Kapoor et al., 2014). (2) Arjunolic acid, an oleanane triterpenoid found in the heartwood of T. arjuna (Roxb. ex DC.) Wight \& Arn., has been demonstrated to contribute to the bioactivities of the plant (Toppo et al., 2018). Also, evidence suggested that T. arjuna (Roxb. ex DC.) Wight \& Arn., bark administration relieved trinitrobenzenesulfonic acid-induced colitis in an animal model by reducing the expression of proinflammatory cytokines and chemokine and decreasing oxidative stress (Cota et al., 2019). Alcoholic extract from the bark of T. arjuna (Roxb. ex DC.) Wight \& Arn., has been demonstrated to protect against picrotoxin in mice by regulating related genes (Chandra Sekhar et al., 2017). The fruit of Terminalia bellirica (Gaertn.) Roxb. is widely documented for its use in treating diseases such as diarrhea, cough, and scorpion-sting, etc. In India, T. bellerica (Gaertn.) Roxb. is used to treat diarrhea based on its antioxidant and antibacterial properties (Pandey et al., 2017). It has been found that extracts of T. bellirica (Gaertn.) Roxb. fruits possess antibacterial activity without having cytotoxicity (Dharmaratne et al., 2018). A recent study showed that the aqueous acetone extract of T. bellirica (Gaertn.) Roxb. fruits attenuate CCl4-induced oxidative stress and liver damage in a rat model (Kuriakose et al., 2017).

Terminalia brownii Fresen. has been used to treat brownerythematous excoriated papules, plaques, and lichenification in the formulation of scented smoke baths (Kibar Ozturk et al., 2018). In traditional medicine in Southeast Asia, the aqueous extract of Terminalia catappa L. leaves are used to treat antipyretic, hemostatic, hepatitis, and liver-related diseases $T$. catappa L. is also used to manage diabetic due to its property of reducing oxidative stress, inflammation, angiogenesis, lipid profile correction, and direct hypoglycemic actions (Behl and Kotwani, 2017). Extracts from the leaves of T. catappa L. has been reported to attenuate the growth of Staphylococcus aureus (ATCC 25923) and Pseudomonas aeruginosa (ATCC 27853) (Allyn et al., 2018). Besides, methanolic extracts from $T$. catappa L. are found to prevent hydrogen peroxide-induced oxidative damage in human fibroblasts (Hs68), thereby can be potentially used to manage skin aging (Huang et al., 2018).

Terminalia chebula Retz. is widely used to treat dementia, constipation, and diabetes in traditional Indian and Iranian medicine (Jokar et al., 2016). Studies showed that T. chebula Retz. has various biological activities, including antimicrobial, antiinflammatory, antioxidant, and antitumor (Zhang et al., 2016). It has been demonstrated that $T$. chebula Retz. fruits are rich in phenolic compounds such as (3) gallic acid, (4) ellagic acid, and (5) corilagin, which hold potent antioxidant, antiinflammatory, cardiotonic, antibacterial, and anticarcinogenic activities (Fan et al., 2015). A randomized placebo-controlled clinical trial indicated that dietary supplementation with a standardized extract of $T$. chebula Retz. fruit (AyuFlex ${ }^{\circledR}$ ) relieves the discomfort in osteoarthritis (Lopez et al., 2017). Also, T. chebula Retz. is a potent cognitive enhancer for amnesia due to its antioxidant activity (Kim et al., 2018). However, the safety assessment regarding the use of $T$. chebula Retz. in amnesia is lacking (Suganthy et al., 2018). Active components such as (4) ellagic acid are reported to play essential roles in the neuroprotective effect of T. chebula Retz. in vivo (Shen et al., 2017). T. chebula Retz. reduces oxidative cell death induced by PC12 and OLN-93 caused by quinolinate, which suggests the neuroprotection and oligoprotection effects of T. chebula Retz. (Sadeghnia et al., 2017). Another study suggested that $T$. chebula Retz. extract attenuates inflammation in microglial cells; therefore, it can be used as a potential anti-inflammatory agent for the treatment of inflammatory diseases of the central nervous system (Rahimi et al., 2018). In addition, the antioxidant activity of $T$. chebula Retz. has been demonstrated in in vitro models in previous studies (Kumar et al., 2018).

It has been reported that the methanolic extract of Terminalia coriacea (Roxb.) Wight \& Arn. (Terminalia coriacea Spreng.) leaves reduce the paw edema and the weights of granulomatous tissue in both acute and chronic in vivo inflammatory models (Khan et al., 2018). Terminalia cunninghamii C.A.Gardner, is a native nut traditionally used by Australian Indigenous peoples for oxidant-related issues (Zhong et al., 2018). The fruit and leaf extracts of Terminalia ferdinandiana Exell, an endemic Australian plant, have been found to possess strong antibacterial activity against various bacterial pathogens (Cheesman et al., 2019). The leaf extract of T. ferdinandiana Exell has a potent growth inhibition effect on plantar malodorproducing bacteria (Mcmanus et al., 2017). It has also been reported that extracts from $T$. ferdinandiana Exell down-regulate the growth of Shewanella sp., which are essential causes of fish spoilage (Wright et al., 2019). Terminalia laxiflora Engl. contains a wide variety of antimycobacterial compounds, including ellagitannins, ellagic acid derivatives, triterpenes, fatty acids, and fatty alcohols (Salih et al., 2018). Evidence indicates that the fungal extract from $T$. laxiflora Engl. affects the NF- $\mathrm{B}$ signaling pathway in K562 myelogenous leukemia cell line (Tawfike et al., 2018). In Mali, Terminalia macroptera Guill. \& Perr. is one of the most widely used plants for malaria in traditional medicine (Pham et al., 2014). The first in vitro T. macroptera Guill. \& Perr. study in 1996 found that the roots and leaves of T. macroptera Guill. \& Perr. hold antibacterial activity (Silva et al., 1996). The safety of the use of T. macroptera Guill. \& Perr. in malaria was further confirmed in the following studies using an in vivo Albino Swiss mice model (Haidara et al., 2018). Terminalia sericea Burch. ex DC. is traditionally used in the 
treatment of stomach ailments, infections, hypertension, and diabetes mellitus (Busisani et al., 2018). Recent studies in medical sciences have revealed that the potential of $T$. sericea Burch. ex DC. includes: antiviral, antibacterial, antitumor, antiinflammatory, antioxidant, and wound healing activities (Fan et al., 2015).

\section{PHYTOCHEMISTRY AND CHEMICAL CONSTITUENTS OF TERMINALIA SP.}

\section{Phytochemistry}

Terminalia sericea Burch. ex DC. is a medicinal plant used mainly to treat diarrhea, sexually transmitted infections, skin rashes, tuberculosis, and other infections. Its biological activities are due to valuable phytochemicals, including triterpenes, alkaloids, and flavonoids (Mongalo et al., 2016). Tannins and polyphenols are two major active components of the plant, which contribute to the bioactivity of Terminalia sp. (Li et al., 2011). Tannins are a kind of polyphenolic compounds that can be classified into three groups based on their structures: hydrolyzable, condensed, and complex tannins (Chang et al., 2019). It is reported that other ingredients, including triterpenoids, flavonoids, and aliphatic compounds, have bioactive properties (Chang et al., 2019). Terminalia catappa L. that has antiHIV features was analyzed for phytochemicals by direct binding assay with mass spectrometry (MS) techniques (Dwevedi et al., 2016). These authors described the presence of tannins, gallotannins, ellagitannins, cyanidin, and flavonoids. More recently, chemical analysis of Terminalia catappa $L$. bark, and leaves performed by Tercas et al. (Terças et al., 2017), reported hydrolyzable tannins (6) punicallin, (7) punicalagin, (3) gallic acid, and flavonoid C-glycosides. These authors have used techniques like gas chromatography coupled to mass spectrometry with electron impact (GC/MS/EI), highperformance liquid chromatography coupled to mass spectrometry "electrospray" ionization in positive mode (HPLC/MS/MS/ESI+) and hydrogen nuclear magnetic resonance (1HNMR). In addition, leaf extract fractions showed antifungal properties against Candida sp.

Recently, Wright and the team explored the antimicrobial properties of Terminalia ferdinandiana Exell (Kakadu plum) extracts against Shewanella sp. growth, the main cause of fish spoilage (Wright et al., 2019). Several compounds were identified by LC-MS analysis that displayed this pharmacological application. The aqueous extract of Terminalia bellirica (Gaertn.) Roxb. from fruits have been reported to contain glycosides, flavonoids, tannins, phenols, saponin, carbohydrates, and proteins (Abraham et al., 2014). Proteins, carbohydrates, and tannins were found in both aqueous, and methanol extracts of Terminalia chebula Retz. leaves. Alkaloids were present in both aqueous and methanol extracts of the fruit, while flavonoids were detected in both aqueous and methanol extracts of all parts of Terminalia chebula Retz. except root (Vemuri et al., 2019). (8) Terflavin B and (9) chebulinic acid were pure compounds isolated from Terminalia chebula Retz. fruits (Cock, 2015). Terminalia schimperiana Hochst. ex Engl. \& Diels (synonym of Terminalia glaucescens Planch. ex Benth.) root bark was reported to contain flavonoids, tannins, steroids, carbohydrates, and terpenoids in nhexane, ethylacetate, and methanol as solvents (Khan et al., 2019). Besides, (10)stigmasterol was a pure compound isolated from the root bark of Terminalia schimperiana Hochst. ex Engl. \& Diels (synonym of Terminalia glaucescens Planch. ex Benth.). The pure compound was a white-yellow crystal, characterized by spectral techniques such as 1H-NMR, 13C-NMR, COSY, HSQC, and HMBC spectral techniques (Khan et al., 2019). Aqueous and methanol extracts of Terminalia grandiflora Benth. nut, fruit, and leaf contained high levels of water-soluble phenolics, moderate to high levels of tannins, and low levels of flavonoids and anthraquinones; while Terminalia carpentariae C.T.White (synonym of Terminalia hadleyana subsp. carpentariae (C.T.White) Pedley), leaf extract showed high levels of watersoluble phenolics and tannins (Wright et al., 2016). Furthermore, the methanol extract of Terminalia grandiflora Benth. nut and Terminalia carpentariae C.T.White [synonym of Terminalia hadleyana subsp. carpentariae (C.T.White) Pedley], leaf exhibited antibacterial activity against Bacillus anthracis with MIC at 155 and $74 \mu \mathrm{g} / \mathrm{mL}$, respectively (Wright et al., 2016). Pure compounds including (11) methyl N-hydroxybenzenecarboximidoate; (12) 1octen-3-ol; (13) 5-hepten-2-one; (14) 6-methyl-, 2-tertbutoxyethanol; (15) 2-ethyl-1-hexanol; (16) dimethyl succinate; (17) isophorone; (18) $\alpha$-citronellol; (19) nonanal; (20) 4oxoisophorone; (21) ethyl benzoate; (22) methyl benzeneacetate; (23) $\alpha$-terpineol; (24) 2-isopropylidene-3-methylhexa-3,5-dienal; (25) lauraldehyde; (26) 2,4-dimethyl-benzaldehyde; (27) 1,3pentanediol; (28) 2,2,4-trimethyl-, 1-isobutyrate; (29) 2,4-di-tertbutylphenol; (30) ethyl para-ethoxybenzoate; and (31) 2,2,4trimethyl-1,3-pentanediol diisobutyrate were detected from the methanol Terminalia carpentariae C.T.White (synonym of Terminalia hadleyana subsp. carpentariae (C.T.White) Pedley), leaf extract using GC-MS headspace analysis (Wright et al., 2016). It has been highlighted that (32) 2-(1,1-dimethylethoxy)ethanol; (33) caryophyllene; (31) 2,2,4-trimethyl-1,3-pentanediol diisobutyrate; and (34) butyl octyl phthalate were detected in Terminalia grandiflora Benth. methanol extract but they were not found in the Terminalia carpentariae C.T.White (synonym of Terminalia hadleyana subsp. carpentariae (C.T.White) Pedley), leaf extract (Wright et al., 2016). Groups of flavonoids, including (35) quercetin, (36) kaempferol, tannins, saponins, and phytosterols presented in the Terminalia catappa L. fruit, have been documented (Cock, 2015). This information indicates varieties of chemical compounds present in Terminalia plant species. This study shows that there are several chemical compounds in Terminalia plant species. As such, assessment and isolation of phytochemicals from Terminalia sp. could hold promise in the discovery of novel new compounds leading to the development of new drugs for the management and control of diseases.

\section{Chemical Constituents of Terminalia sp.}

The stem bark of T. arjuna (Roxb. ex DC.) Wight \& Arn. contains high levels of antioxidant compounds, including glycosides, flavonoids, tannins, and inorganic minerals. 
Amongst the terpenoids reported in T. arjuna (Roxb. ex DC.) Wight \& Arn. bark is (37) $\beta$-sitosterol, (38) terminic acid (Table 1) (Anjaneyulu and Prasad, 1982), (39) terminoside A (Ali et al., 2003a; Ali et al., 2003b), and (40) arjunaphthanoloside (Table 1). (39) Terminoside A and (40) arjunaphthanoloside are particularly interesting due to their reported therapeutic activities. Terminoside A inhibits the production of nitric oxide and decreases the levels of nitric oxide synthase in macrophages stimulated by lipopolysaccharides, and arjunaphthanoloside has strong antioxidant activity (Ali et al., 2003a; Ali et al., 2003b). T. arjuna (Roxb. ex DC.) Wight \& Arn. bark also contains very high levels of antioxidant flavonoid compounds compared to other plants (Nair et al., 1996; Nair and Nagar, 1997). These flavonoids include (41) arjunolone, (42) bicalein, flavones, (36) kampferol, (43) pelorgonidin, (35) quercetin, (Sharma et al., 1982), and (48) luteolin, (Pettit et al., 1996). There is evidence that similar bioflavonoids prevent oxidation of LDL cholesterol through its free radical scavenging activity (Fuhrman and Aviram, 2001), inhibit endothelial activation (Carluccio et al., 2003) and inhibit platelet aggregation (Ruf, 1999). They also have cyclooxygenase inhibitory activity and, therefore, can prevent thrombosis (Ruf, 1999). Kuo et al. (2005), reported (44) castalagin, (8) terflavin, and (45) terchebulin while Lin et al. (2000), reported (44) castalagin from T. arjuna (Roxb. ex DC.) Wight \& Arn.

T. ferdinandiana Exell fruit is the main source of vitamin $\mathrm{C}$, in the health food, cosmetic, and pharmaceutical industries. However, T. ferdinandiana Exell fruit also contains many other compounds that also contribute to its high antioxidant activity (Netzel et al., 2007; Konczak et al., 2010). Although many of these compounds have not been identified yet, $T$. ferdinandiana Exell fruit has been shown to contain benzoic acids, flavanols, or flavanones (Konczak et al., 2010). T. ferdinandiana Exell fruit is a good source of (3) gallic acid and (4) ellagic acid (Table 2) (Cunningham et al., 2009), which also demonstrates strong antioxidant activity in vitro (Ohno et al., 1999; Losso et al., 2004). T. ferdinandiana Exell fruit is also very rich in (36) chlorophyll a and (47) chlorophyll b (Table 2), which have previously been shown to be capable of quenching oxidative stress. Lipophilic T. ferdinandiana Exell fruit extracts are also rich in (48) luteolin and with (49) vitamin E (Table 2) and vitamin E analogs (Konczak et al., 2010). (50) Hesperitin (Table 2), as well as the glycosides (36) kaempferol, (48) luteolin, and (35) quercetin (Table 2), are some of the other antioxidants present in T. ferdinandiana Exell fruit (Konczak et al., 2010). T. ferdinandiana Exell fruit is also a good source of the minerals magnesium, zinc, calcium, potassium, sodium, iron, phosphorous, manganese, copper, and molybdenum (Konczak et al., 2010).

\section{PHARMACOLOGICAL EFFECTS OF ACTIVE CONSTITUENTS FROM TERMINALIA SP.}

There is a rising global interest in ethno-pharmacological studies on plants that have been traditionally used to alleviate a myriad of diseases (World Health Organization, 1999; World Health
Organization, 2018). Due to the increasing number of people with non-communicable diseases, which represent about $70 \%$ of deaths in the world, the search for new pharmacological agents started to evaluate the medicinal plants mentioned in the pharmacopeias of different countries (World Health Organization, 1999; World Health Organization, 2018). In this sense, Terminalia sp. has been the center of attention of many studies, which aim to evaluate and validate the therapeutic potential based on its ethnobotanical use. As Terminalia species can be found in many countries, the ethnobotanical information on their medicinal use is diverse; for example fruits, leaves, and stem bark of different Terminalia sp. has been reported to be used as a remedy to treat geriatric diseases, memory improvement, abdominal and back pain, cough, and colds, conjunctivitis, diarrhea, fever, headache, heart disorders, inflammation, leprosy, pneumonia, sexually transmitted diseases, urinary disorders, among others (Maulik and Katiyar, 2010; Maulik and Talwar, 2012; Cock, 2015; Afshari et al., 2016). Medicinal plants have long been known to be rich sources of bioactive compounds. Terminalia sp. belongs to the family Combretaceae, has several biological activities, including antibacterial, antifungal, as well as antiparasitic. In addition, the extracts from the plant species showed antidiabetic, anticancer, and antioxidant activity.

Antidiabetic and Antiobesity Studies of Terminalia sp.

The International Diabetes Federation states that type-2 diabetes is a chronic condition that occurs when there are raised levels of plasma glucose, attributed to insufficient/lack production of insulin, or because the body cannot use the insulin it produces (International Diabetes Federation, 2019). It is estimated that around 463 million people live with diagnosed diabetes, and this number is expected to rise to 700 million by 2045. The first line of treatment of diabetes is a combination of exercise, changes in lifestyle, dietary modification, and drug prescription; sulfonylureas and meglitinides, metformin, PPAR antagonists (like thiazolidinediones), $\alpha$-glucosidase inhibitors, among others (Defronzo et al., 2015). However, people from low- and middle-income countries have restricted access to pharmaceutical treatment due to economic restraints; thus, they sometimes use alone or in combination different medicinal plants to treat hyperglycemia, diabetes and some of its complications (World Health Organization, 1999; Mata et al., 2013). Studies regarding the potential antidiabetic properties of Terminalia sp. have been consistent from the last 10 years (according to the Web of Science and Scopus databases); however, it is important to mention that most of these reports represent only in vitro studies. This section aims to gather recent studies with Terminalia sp. and their potential antidiabetic activity.

A study by Yang et al. (2013), evaluated fruits from two different Terminalia sp., T. chebula Retz., and T. bellerica (Gaertn.) Roxb., and an Ayurvedic formulation from the two species. The authors showed that the compounds (53) chebulagic acid; (5) corilagin; (54) 2,3,6-tri-O-galloyl- $\beta$-glucose; (55) $1,2,3,6$-tetra-O-galloyl- $\beta$-D-glucose found in $T$. bellerica (Gaertn.) Roxb. and the formulation enhanced the signaling of 8 Peroxisome proliferator-activated receptors (PPAR $\alpha$ and 
TABLE 1 | Chemical Constituents of T. arjuna (Roxb. ex DC.) Wight \& Arn.

Compound No. Chemical Constituents name

(37)

38)

Terminic acid

(48)

Luteolin

(44)

Castalagin

(8)

Terflavin

(45)

Terchebulin
Structure
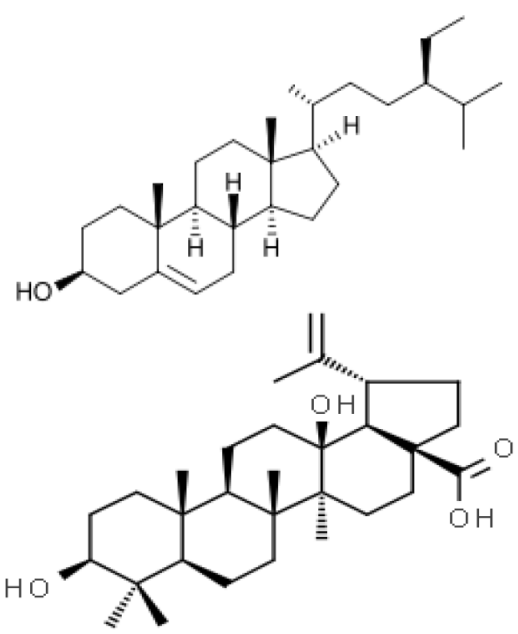<smiles>O=c1cc(-c2ccc(O)c(O)c2)oc2cc(O)cc(O)c12</smiles>

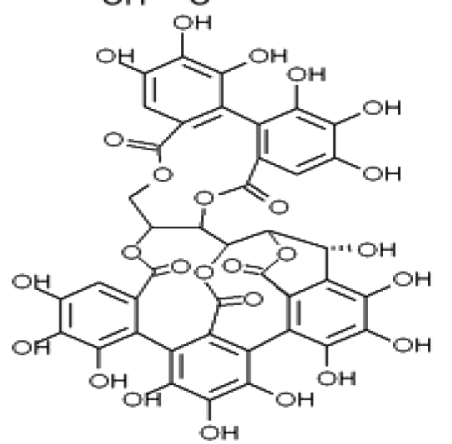<smiles>O=C1OC2=C(O)C(O)=C(c3c(C(=O)OC4C(COC(=O)c5cc(O)c(O)c(O)c5)OC(O)C(O)C4O)cc(O)c(O)c3O)C3=C1C=C(O)C(O)=C1COC2=C13</smiles>

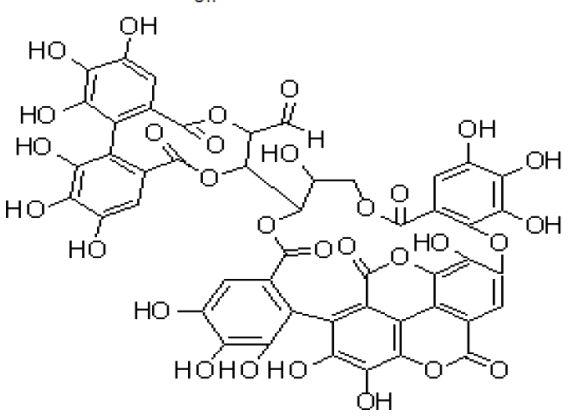

Reference

(Anjaneyulu and Prasad, 1982)

(Anjaneyulu and Prasad, 1982)

(Pettit et al., 1996)

(Kuo et al., 2005)

(Kuo et al., 2005)

(Kuo et al., 2005) 
TABLE 1 | Continued

Compound No.

Chemical Constituents name

Structure

Reference

(6)

Punicallin

(43)

Pelorgonidin

(42)

Bicalein

(41)

Arjunolone

(39)

Terminoside A

(Lin et al., 2000)

(Sharma et al., 1982)<smiles>Oc1ccc(-c2[o+]c3cc(O)cc(O)c3cc2O)cc1</smiles>

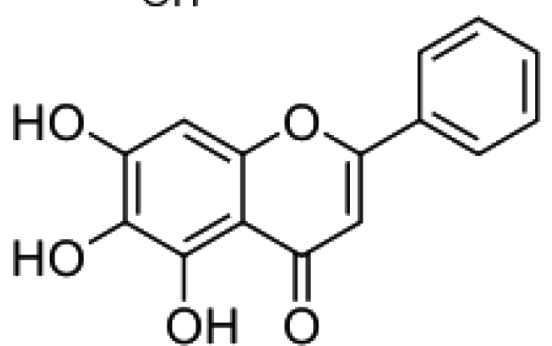<smiles>COc1cc2oc(-c3ccc(O)cc3)cc(=O)c2cc1O</smiles>

(Sharma et al., 1982)

(Sharma et al., 1982)

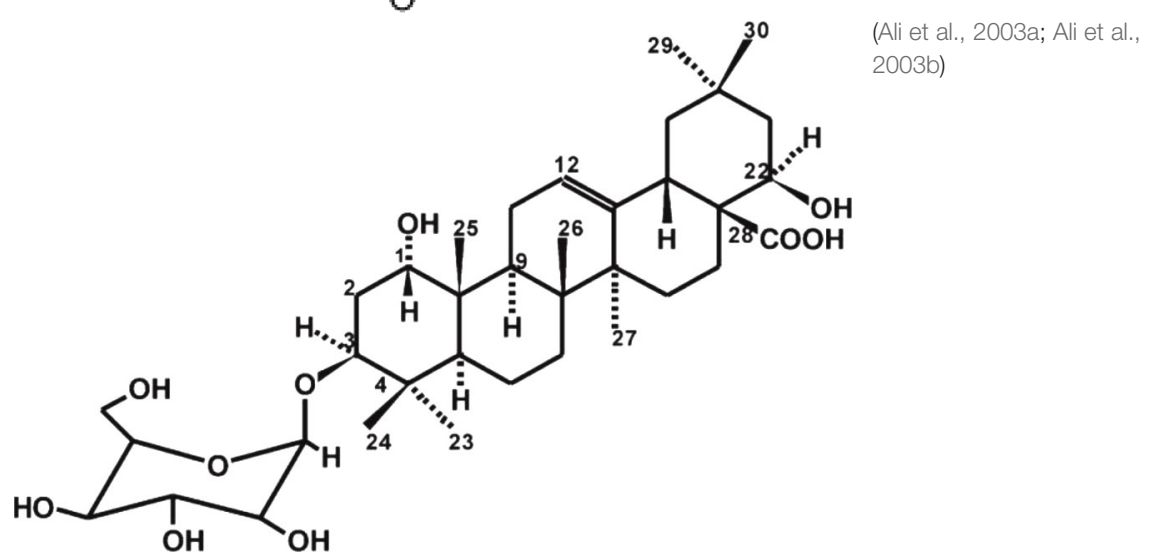


TABLE 2 | Chemical Constituents of T. ferdinandiana Exell.

Compound No.

(51)

(3)

Gallic acid

(35)

(52)

(4)

(48)

(36)

(50)

(46)

Quercetin

Ellagic acid

Luteolin

Kaempferol

Hesperitin
Chemical Constituents name

Ascorbic acid

Chlorophyll a $\alpha$-tocopherol
Structure

Reference

(Netzel et al., 2007; Konczak et al., 2010)

(Cunningham et al., 2009)<smiles>O=C(O)c1cc(O)c(O)c(O)c1</smiles>

(Konczak et al., 2010)

(Konczak et al., 2010)<smiles>Cc1c(C)c2c(c(C)c1O)CC[C@@](C)(CCC[C@H](C)CCC[C@H](C)CCCC(C)C)O2</smiles>

(Cunningham et al., 2009)

(Konczak et al., 2010)<smiles>O=c1c(O)c(-c2ccc(O)c(O)c2)oc2cc(O)cc(O)c12</smiles><smiles>O=c1cc(-c2ccc(O)c(O)c2)oc2cc(O)cc(O)c12</smiles><smiles>O=c1c(O)c(-c2ccc(O)cc2)oc2cc(O)cc(O)c12</smiles><smiles>COc1ccc([C@H]2CC(=O)c3c(O)cc(O)cc3O2)cc1O</smiles> 
TABLE 2 | Continued

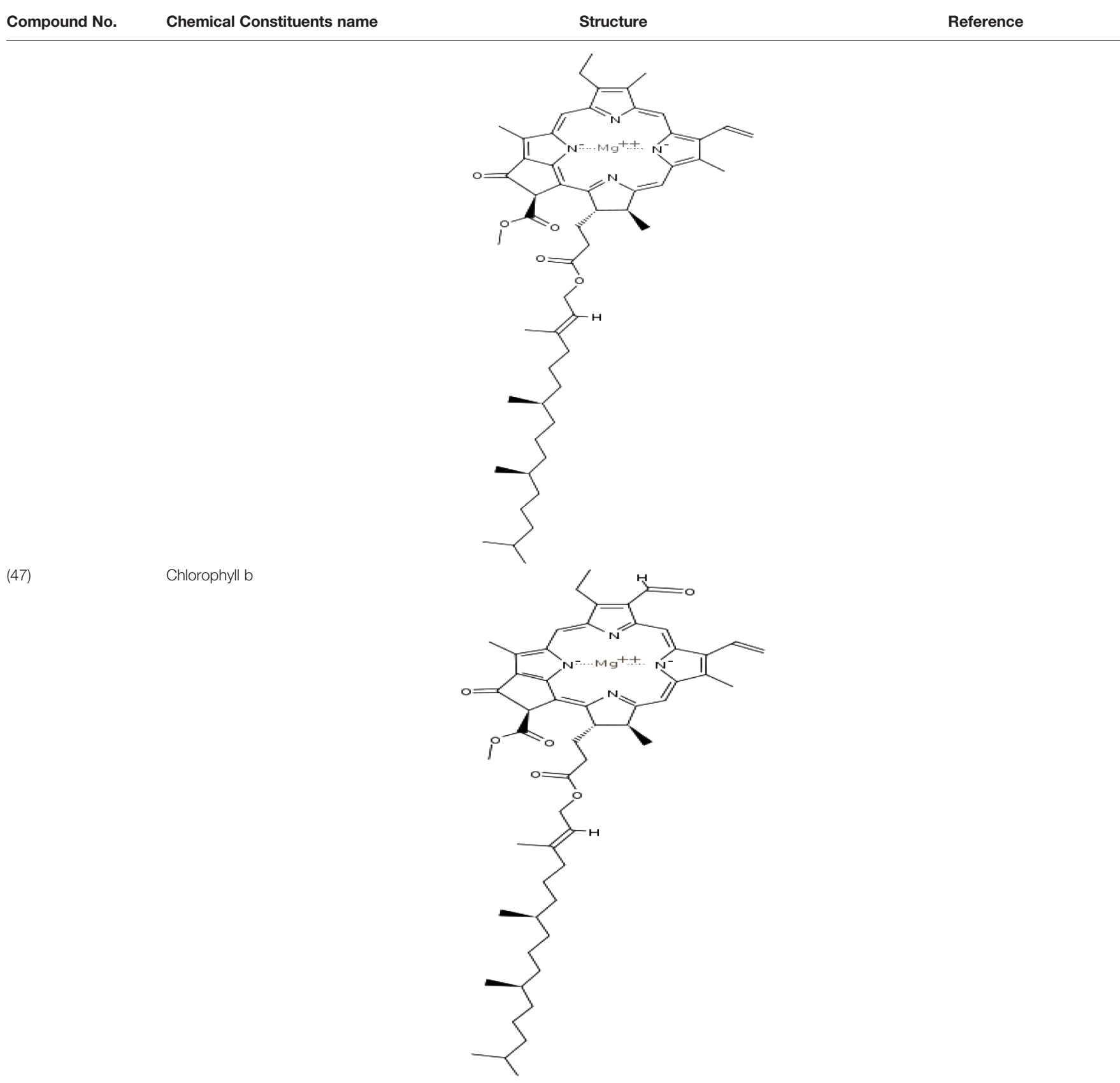

PPAR $\gamma)$. A structure-dependent effect was shown when only the gallotannins, (56) 1,2,3,6-tetra-O-galloyl- $\beta$-D-glucose and (57) 1,2,3,4,5-penta-O-galloyl- $\beta$-D-glucose enhanced up to 9.92-fold the cellular glucose uptake in HepG2 cells and inhibited the rosiglitazone-induced adipogenesis (Yang et al., 2013). A similar report by Shyni et al. (2014), showed the potential of (53) chebulagic acid to enhance glucose transport in adipocytes using 3T3-L1 preadipocytes to elucidate its PPAR $\gamma$ agonistic effect. To achieve this, the authors used (53) chebulagic acid from Terminalia chebula Retz. using 3T3-L1 cells at concentrations of 10, 50, and $100 \mu \mathrm{M}$. The importance of research for PPAR $\gamma$ antagonists from plant origin is to elucidate active molecules that partially inhibit these molecules, without the adverse side effects caused by conventional PPAR $\gamma$ antagonists (Shyni et al., 2014). The authors concluded that (53) chebulagic acid from T. chebula Retz. enhanced the expression of C/EBP $\alpha$, a PPAR $\gamma$ target in adipocytes, but not at the extent of rosiglitazone.

Mopuri et al. (2015), evaluated a different Terminalia species, T. paniculata Roth from India. The study tested the antiobesity effect of ethanolic extracts from barks of $T$. paniculata Roth on male Sprague-Dawley rats. Of the evaluated parameters, Terminalia extracts reduced body weight, lean mass, total fat, fat percentage, decreased glucose blood levels at $60 \mathrm{~min}$, reversed insulin resistance, and lowered serum cholesterol, triglycerides, 
and low-density lipoproteins. The authors concluded that the potential antiobesity effect of barks from $T$. paniculata Roth might be attributed to the down-regulation of the expression of lipogenic genes and leptin; also, and up-regulation of adiponectin and AMPK-1 $\alpha$. The potential hypolipemic effect of Terminalia chebula Retz. has been assessed by Reddy et al. (2015). The authors used methanolic bark extracts of T. chebula Retz. at concentrations of 5, 50, 300, and $2000 \mathrm{mg} / \mathrm{kg}$. The toxicity of these extracts was evaluated using 8-12 weeks old female rats at the doses mentioned above. Reddy et al. (2015), report no observed toxicity effect with any of the tested concentrations. Furthermore, the administration of T. chebula Retz. bark extract at $600 \mathrm{mg} / \mathrm{kg}$ significantly caused a hypolipidemic effect in high-cholesterol hyperlipidemic rats, which was evidenced by increased serum high-density lipoprotein cholesterol levels.

Another mechanism in which Terminalia sp. has shown its antiobesity potential is through the inhibition of glucose metabolic enzymes such as $\alpha$-glucosidase. For instance, Pham et al. (2014), evaluated Terminalia macroptera Guill. \& Perr. extracts glucosidase inhibitory capacity. Chromatographic analysis showed the presence of the polyphenols (53) chebulagic acid, (73) chebulic acid trimethyl ester, (71) corilagin, (70) methyl gallate, (74) narcissin, and (118) rutin. Methanol, ethyl acetate, and butanol Terminalia sp. extracts were outstanding inhibitors of $\alpha$-glucosidase with $\mathrm{IC}_{50}$ values of $0.47,0.4$, and $0.4 \mu \mathrm{M}$, respectively. Moreover, (53) chebulagic acid isolated from extracts showed an inhibitory rate with $\mathrm{IC}_{50}$ values of $0.05 \mu \mathrm{M}$. Similarly, Nguyen et al. (2016), studied the enzymatic inhibitory potential of extracts of trunk-bark of three Terminalia species, $T$. alata Roth, T. bellerica (Gaertn.) Roxb. and T. corticosa Pierre ex Laness.

Terminalia species showed inhibitory activity against $\alpha$ amylase and $\alpha$-glucosidase, and lowered fasting blood glucose in streptozotocin-induced diabetic rats. T. bellerica (Gaertn.) Roxb. extracts showed the most potent inhibitory activity against $\alpha$-glucosidase, followed by $T$. corticosa Pierre ex Laness, and $T$. alata Roth, with $\mathrm{IC}_{50}$ values of $0.41,1.42$, and $\geq$ $4 \mathrm{mg} / \mathrm{mL}$, respectively. The authors found a positive correlation between the polyphenolic content of Terminalia species (as evaluated by spectrophotometry) and the $\alpha$-glucosidase inhibitory capacity of the extracts. Moreover, Makihara et al. (2012), evaluated hot water extracts of fruits from Terminalia bellirica (Gaertn.) Roxb. on obesity-related disorders. The authors suggest that Terminalia sp. has a preventive effect on obesity, insulin resistance, and hyperlipidemia in spontaneously obese type 2 diabetic mice. Terminalia sp. treatment does not affect food intake; however, it slightly suppressed body weight gain (Makihara et al., 2012). Moreover, T. bellerica (Gaertn.) Roxb. treatment significantly suppressed in a dose a timedependent manner, the accumulation of visceral and subcutaneous fat after the 7 th week. Terminalia treatment also improved plasma and hepatic lipid levels, as it was observed by the decreased LDL/HDL ratio from 0.28 to 0.23 in Terminalia sp. at $3 \%$ treated mice. The authors managed to identified that gallic acid might be the responsible active compound for the inhibition of lipid absorption, as it is a potent pancreatic lipase inhibitor. As a follow-up of the study, Makihara et al. (2016), studied the anti-

TABLE 3 | Summarization of the antiobesity and antidiabetic studies of different Terminalia species.

\begin{tabular}{|c|c|c|c|}
\hline Terminalia sp. & Mechanism/Mode of action & Phytochemicals (compound no.) & Reference \\
\hline T. bellerica (Gaertn.) Roxb. & $\begin{array}{l}\text { Enhancement of PPAR } \alpha \text { and PPAR } \gamma \text {, increased insulin- } \\
\text { stimulated glucose uptake. 1,2,3,6-tetra-O-galloyl- } \beta \text {-D- } \\
\text { glucose showed the most potent increased in cellular }\end{array}$ & $\begin{array}{l}\text { (53) Chebulagic acid; (55) 1,2,3,6-tetra-O-galloyl- } \beta \text {-D- } \\
\text { glucose; (57) 1,2,3,4,5-penta-O-galloyl- } \beta \text {-D-glucose; } \\
\text { (59) daucosterol, (3) gallic acid }\end{array}$ & (Yang et al., 2013) \\
\hline T. chebula Retz. & glucose uptake & $\begin{array}{l}\text { (59) Arjunetin; (60) arjungenin; (61) arjunglucosides; } \\
\text { (62) (63) chebuloside II; (64) shikimic acid }\end{array}$ & \\
\hline $\begin{array}{l}\text { T. bellerica (Gaertn.) Roxb. } \\
\text { and } T \text {. chebula Retz. }\end{array}$ & & (5) Corilagin; (54) 2,3,6-tri-O-galloyl- $\beta$-glucose & \\
\hline T. chebula Retz. & $\begin{array}{l}\text { mRNA expression of C/EBP- } \alpha \text {, a target gene for } \\
\text { PPARy, increased with chebulagic acid treatment }\end{array}$ & (53) Chebulagic acid & (Shyni et al., 2014) \\
\hline T. paniculata Roth & $\begin{array}{l}\text { Reduced expression of lipogenic genes (FAS, SREBP- } \\
\text { 1c, PAPRy, and leptin), up-regulation of adiponectin and } \\
\text { AMPK-1 } \alpha\end{array}$ & $\begin{array}{l}\text { (4) Ellagic acid; (65) 2',5',5,7-tetramethoxy-8- } \\
\text { methylflavanone; (66) 3,3'-di-O-methyl ellagic acid; (2) } \\
\text { arjunolic acid; (67) galloylarjunolic acid; (68) } \\
\text { termilignan; (69) betulinic acid }\end{array}$ & (Mopuri et al., 2015) \\
\hline T. chebula Retz. & $\begin{array}{l}\text { Increased levels of serum high-density lipoprotein } \\
\text { cholesterol levels in hypercholesterolemic rats }\end{array}$ & Not identified & (Reddy et al., 2015) \\
\hline T. macroptera Guill. \& Perr. & The inhibitory capacity of $\alpha$-glucosidase & $\begin{array}{l}\text { (64) Shikimic acid, (70) methyl gallate, (71) coriliagin, } \\
\text { (53) chebulagic acid, (72) chebulagic acid trimethyl } \\
\text { ester, (118) rutin, (74) narcissin }\end{array}$ & (Pham et al., 2014) \\
\hline T. alata Roth & $\begin{array}{l}\text { The inhibitory capacity of } \alpha \text {-glucosidase }\left(\mathrm{IC}_{50} \geq 4 \mathrm{mg} /\right. \\
\mathrm{mL})\end{array}$ & Not identified & (Nguyen et al., 2016) \\
\hline T.belirica (Gaertn.) Roxb. & Inhibitory capacity of $\alpha$-glucosidase $\left(\mathrm{IC}_{50} \geq 0.41 \mathrm{mg} / \mathrm{mL}\right)$ & Not identified & \\
\hline $\begin{array}{l}\text { T. corticosa Pierre ex } \\
\text { Laness }\end{array}$ & $\begin{array}{l}\text { The inhibitory capacity of } \alpha \text {-glucosidase }\left(\mathrm{IC}_{50} \geq 1.42\right. \\
\mathrm{mg} / \mathrm{mL})\end{array}$ & Not identified & \\
\hline T. bellirica (Gaertn.) Roxb. & $\begin{array}{l}\text { Preventive effect on obesity, insulin resistance, and } \\
\text { hyperlipidemia in obese type- } 2 \text { diabetic mice }\end{array}$ & (3) Gallic acid & (Makihara et al., 2012) \\
\hline T. bellirica (Gaertn.) Roxb. & $\begin{array}{l}\text { Reduction in plasma glucose levels, potentiation of } \\
\text { insulin secretion from } \beta \text {-cells }\end{array}$ & (58) Octyl gallate & $\begin{array}{l}\text { (Latha and Daisy, } \\
\text { 2013) }\end{array}$ \\
\hline
\end{tabular}




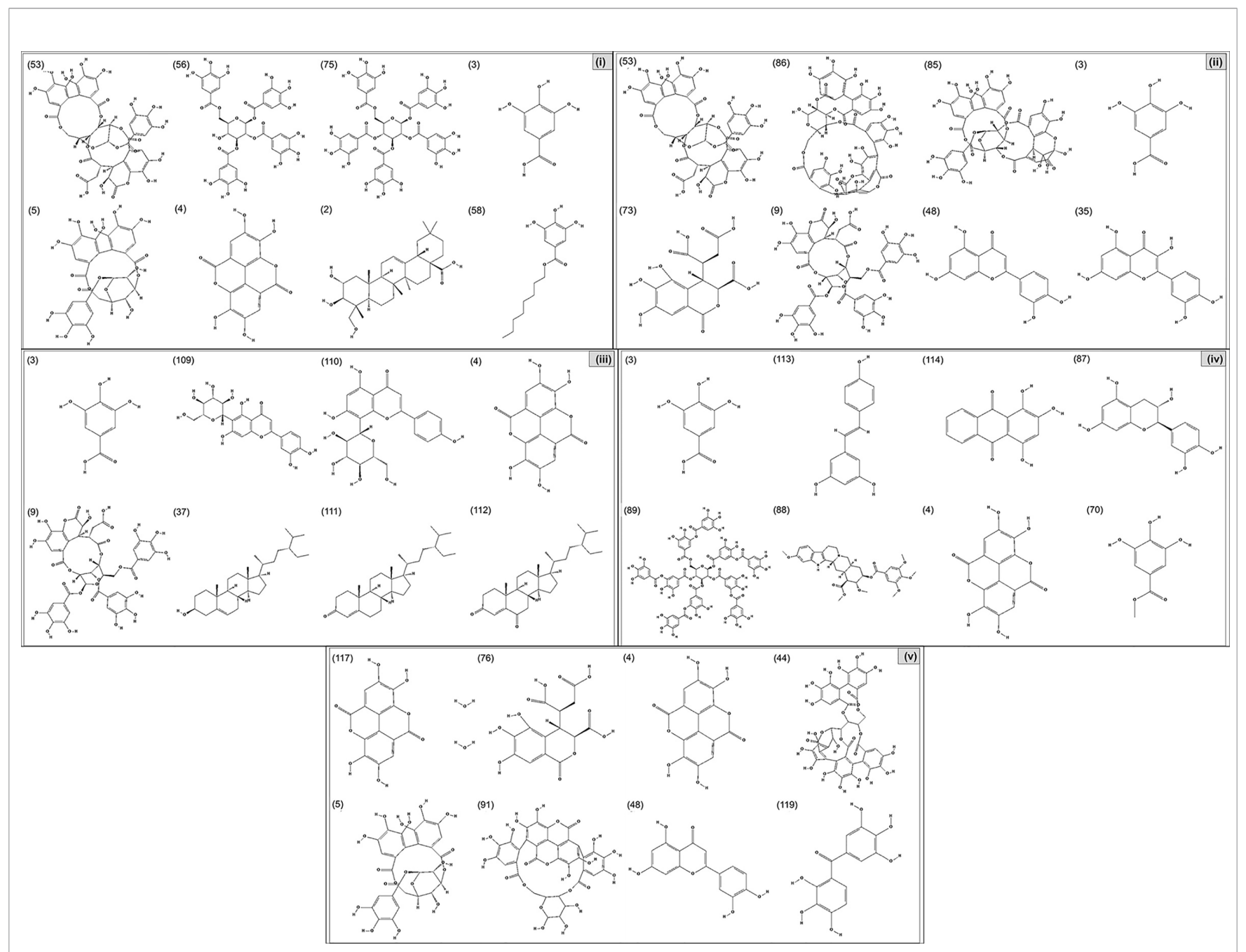

FIGURE 4 | (i) Compounds from Terminalia species that have been studied for their antidiabetic and antiobesity properties. (53) Chebulagic acid, (56) 1,2,3,6-tetra-

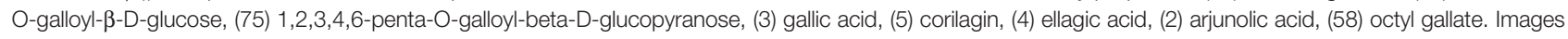
from the National Center for Biotechnology Information, 2019a; National Center for Biotechnology Information, 2019c; National Center for Biotechnology Information, 2019g; National Center for Biotechnology Information, 2019i; National Center for Biotechnology Information, 2019k; National Center for Biotechnology Information, 2019m; National Center for Biotechnology Information, 2019r; National Center for Biotechnology Information, 2019s; (ii) Compounds from Terminalia species that have been studied for their antiproliferative properties. (53) Chebulagic acid, (86) punicalagin, (85) geraniin, (3) gallic acid, (73) chebulic acid, (9) chebulinic acid, (48) luteolin, (35) quercetin. Images from the National Center for Biotechnology Information, 2019c; National Center for Biotechnology Information, 2019d; National Center for Biotechnology Information, 2019f; National Center for Biotechnology Information, 2019h; National Center for Biotechnology Information, 2019m; National Center for Biotechnology Information, 2019n; National Center for Biotechnology Information, 2019p; National Center for Biotechnology Information, 2019v; (iii) Compounds from Terminalia species that have been studied for their antiinflammatory properties. (3) Gallic acid, (109) luteolin-6-C-glucoside, (110) vitexin, (4) ellagic acid, (9) chebulinic acid, (37) $\beta$-sitosterol, (111) $\beta$-sitostenone, and (112) stigmast-4-ene-3,6-dione. Images adapted from National Center for Biotechnology Information, 2019aa; National Center for Biotechnology Information, 2019ad; National Center for Biotechnology Information, 2019b; National Center for Biotechnology Information, 2019f; National Center for Biotechnology Information, 2019k; National Center for Biotechnology Information, 2019m; National Center for Biotechnology Information, 2019o; National Center for Biotechnology Information, 2019z; (iv) Compounds from Terminalia species that have been studied for their antioxidant properties. (3) gallic acid, (113) resveratrol, (114) purpurin, (87) catechin, (89) tannic acid, (88) reserpine, (4) ellagic acid, (70) methyl gallate. Images adapted from National Center for Biotechnology Information, 2019ab; National Center for Biotechnology Information, 2019e; National Center for Biotechnology Information, 2019k; National Center for Biotechnology Information, 2019m; National Center for Biotechnology Information, 2019q; National Center for Biotechnology Information, 2019u; National Center for Biotechnology Information, 2019w; National Center for Biotechnology Information, 2019x; National Center for Biotechnology Information, 2019y; (v) Compounds from Terminalia species that have been studied for their antimicrobial properties. (117) ellagic acid dihydrate, (76) chebulic acid, (4) ellagic acid, (44) castalagin, (5) corilagin, (91) punicalin, (48) Iuteolin, (119) exifone. Images adapted from National Center for Biotechnology Information, 2019ac; National Center for Biotechnology Information, 2019d; National Center for Biotechnology Information, 2019i; National Center for Biotechnology Information, 2019j; National Center for Biotechnology Information, 2019k; National Center for Biotechnology Information, 2019|; National Center for Biotechnology Information, 2019p; National Center for Biotechnology Information, 2019t. 
TABLE 4 | Summarization of the anticancer studies with different Terminalia species.

\begin{tabular}{|c|c|c|c|}
\hline Terminalia sp. & Mechanism/Mode of action & Phytochemicals (compound no.) & Reference \\
\hline T. chebula Retz. & $\begin{array}{l}\text { Inhibition of cell proliferation by induction of } \\
\text { apoptosis and cell-cycle arrest by regulation of } \\
\text { the Bcl- } 2 \text { family }\end{array}$ & Not identified & (Wang et al., 2015) \\
\hline T. ferdinandiana Exell & $\begin{array}{l}\text { Antiproliferative activity against carcinoma cell } \\
\text { proliferation }\end{array}$ & $\begin{array}{l}\text { Ethyl acetate extracts: (5) corilagin, (48) luteolin, (76) } \\
\text { chebulic acid, (90) protocatechuic acid, (118) rutin, (91) } \\
\text { punicalin, (53) chebulagic acid }\end{array}$ & (Shalom and Cock, 2018) \\
\hline T. citrina (Gaertn.) Roxb. & $\begin{array}{l}\text { Antiproliferative and antiestrogenic activity against } \\
\text { MCF-7 cells; antiproliferative activity against T47D } \\
\text { cell lines }\end{array}$ & $\begin{array}{l}\text { (91) Terminaloside A, (77) terminaloside B, (80) } \\
\text { terminaloside } \mathrm{C},(92) \text { terminaloside } \mathrm{D},(93) \text { terminaloside } \mathrm{E} \text {, } \\
\text { (81) terminaloside F, (78) terminaloside } \mathrm{G},(94) \\
\text { terminaloside } \mathrm{H},(82) \text { terminaloside I, (95) terminaloside J, } \\
\text { (96) terminaloside K, (97) 2-epiterminaloside D, and (79) 6- } \\
\text { epiterminaloside K }\end{array}$ & (Muhit et al., 2016) \\
\hline T. catappa L. & $\begin{array}{l}\text { Inhibition of cellular migration and invasion in } \\
\text { human HeLa and SiHa cervical cancer cell lines }\end{array}$ & Not identified & (Lee et al., 2018) \\
\hline T. chebula Retz. & & $\begin{array}{l}\text { (86) Punicalagin, (85) geraniin, (76) chebulic acid, (9) } \\
\text { chebulinic acid, (3) gallic acid }\end{array}$ & (Lee et al., 2016) \\
\hline T. chebula Retz. & $\begin{array}{l}\text { Inhibition of the TNF-induced necroptotic cell } \\
\text { death }\end{array}$ & $\begin{array}{l}\text { (3) Gallic acid (hypothesized active compound), (85) } \\
\text { geraniin, (76) chebulic acid, (86) punicalagin, (9) chebulinic } \\
\text { acid, (53) chebulagic acid }\end{array}$ & \\
\hline T. chebula Retz. & $\begin{array}{l}\text { Chebulagic acid induces G1 arrest and induces } \\
\text { apoptosis in retinoblastoma Y79 cells }\end{array}$ & (53) Chebulagic acid & (Kumar et al., 2014) \\
\hline T. bellerica (Gaertn.) Roxb. & $\begin{array}{l}\text { Induced apoptosis in human lung A459 and } \\
\text { human breast MCF-7 cancer cell lines }\end{array}$ & $\begin{array}{l}\text { (35) Quercetin, (3) gallic acid, (87) catechin, (89) tannic } \\
\text { acid, (88) reserpine }\end{array}$ & (Ghate et al., 2014) \\
\hline
\end{tabular}

obesity mechanism of gallic acid of fruits from Terminalia bellirica (Gaertn.) Roxb. by evaluating adipocyte3 differentiation using mouse 3T3-L1 cells. Gallic acid at concentrations of 10$30 \mu \mathrm{M}$ enhanced the expression and secretion of adiponectin via adipocyte differentiation, which is enhanced by $T$. bellerica (Gaertn.) Roxb. extracts. Gallic acid enhanced the expression of the mRNA encoding for the marker of adipocyte differentiation Fabp4.

Bioassay-guided fractionation of Terminalia bellirica (Gaertn.) Roxb. fruit extracts by Latha and Daisy (2013), isolated and identified (58) octyl gallate by $1 \mathrm{H}$ and 13C NMR, IR, and mass spectrometry. Also, they reported it as the active compound in T. bellerica (Gaertn.) Roxb. responsible for its bioactive potential when examined for its antidiabetic potential. An in vivo assay showed that (58) octyl gallate at concentrations of 5,10 , and $20 \mathrm{mg} / \mathrm{kg}$ significantly reduced plasma glucose in a dose-dependent manner in diabetic mice. However, the plasma glucose of normal rats treated with the highest (58) octyl gallate concentration was not altered, indicating a normoglycemic effect. This may be attributed to improved proinsulin processing and potentiation of insulin secretion and release from $\beta$-cells. This effect is hypothesized as (58) octyl gallate has been reported as a calcium channel and cAMP modulator (Latha and Daisy, 2013). The summary of different Terminalia species with antiobesity and antidiabetic potential is given in Table 3 . In conclusion, phytochemicals such as phenolic compounds, polyphenols, and terpenoids are among the active compounds in Terminalia species with antidiabetic and antiobesity potential [Figure 4(i)].

\section{Anticancer Studies of Terminalia sp.}

The World Health Organization states that "cancer is a large group of diseases that can start in almost any organ when abnormal cells grow uncontrollably" (World Health
Organization, 2019). Cancer is a non-communicable disease and the second leading cause of death worldwide, only after cardiovascular diseases. In 2018, around 9.6 million people died from cancer, and up to 300,000 new cases are registered per year. Moreover, cancer causes a big economic burden on patients and health care systems with an estimated world annual cost of US\$ 1.16 trillion in 2010 (World Health Organization, 2019). Unfortunately, due to the high cost of cancer treatment, people from low and middle-income countries cannot afford conventional drugs and sometimes turn to the use of medicinal plants or extracts from plants for their treatment. Additionally, several chemotherapy agents that are currently used are of plant origin. Thus, studies are still evaluating natural products from new plant species searching for potential anticancer agents. Recent information from the Scopus and Web of Science databases (2010-2020) indicates an ongoing interest in evaluating the anticancer potential of Terminalia species.

Wang et al. (2015), evaluated the antiproliferative potential of aqueous extracts of Terminalia chebula Retz. using human lung cancer A and mouse lung cancer LLC cell lines. This report isolated five different fractions from the aqueous extracts and shows that $T$. chebula Retz. extracts inhibit cell proliferation by inducing apoptosis and cell-cycle arrest by regulating the mitochondrial pathway mediated by proteins of the $\mathrm{Bcl}-2$ family, inducing the PARP cleavage, and promoting cytochrome $\mathrm{c}$ release into the cytoplasm. T. ferdinandiana Exell from Australia was studied by Shalom and Cock (2018); the authors evaluated methanolic, aqueous, ethyl acetate, chloroform, and hexane extracts. It was shown that all fruit extracts significantly had an inhibitory effect against Caco- 2 cells; though, only methanol and aqueous fruit extracts showed HeLa antiproliferative activity. The antiproliferative potential as determined in $\mathrm{IC}_{50}$ values showed that the most potent 
extracts were ethyl acetate $\left(\mathrm{IC}_{50}=6 \mu \mathrm{g} / \mathrm{mL}\right)$ against MC3T3-E1 cells, methanolic extracts against MC3T3-E1 $\left(\mathrm{IC}_{50}=40 \mu \mathrm{g} / \mathrm{mL}\right)$, and Jeg-3 $\left(\mathrm{IC}_{50}=147 \mu \mathrm{g} / \mathrm{mL}\right)$ cells. As ethyl acetate extracts were the most antiproliferative, the authors only evaluated the phytochemical composition of these samples. It was revealed that this extract is rich in (5) corilagin, (48) luteolin, and (76) chebulic acid. Further studies showed that the mechanism of action was through elevation of the caspase- 3 activity, indicating an apoptosis-induced effect.

(58) Octyl gallate and (3) gallic acid isolated from T. bellirica (Gaertn.) Roxb. had an antiproliferative effect against MCF-7 $\left(\mathrm{IC}_{50}=40 \mu \mathrm{g} / \mathrm{mL}\right)$ and MDA-MB-231 cell lines through induced apoptosis by altering the expression of the cell regulators cyclin D1, D3, CDK-4, CDK-6, p18 INK4, p21Waf-1, and p27 KIP. The extracts downregulated the overexpressed cyclin D/CDKs, molecules involved in the progression of the cell cycle through G1 to $S$ phase, which contributes to the induction of apoptosis. Moreover, docking studies confirmed that (58) octyl gallate and (3) gallic acid have a strong binding affinity with the cell cycle regulators by hydrogen bonds. Another group of compounds with antiproliferative potential is furfuran lignans from $T$. citrina (Gaertn.) Roxb. Muhit et al. (2016), tested these furfuran lignans at concentrations of $0.01,0.1,1.0$, and $10 \mu \mathrm{M}$, and the compounds (77) terminaloside $B$ and (78) terminaloside $G$ exhibited antiproliferative effect for MCF-7 and T47D cell lines with suppression of nearly $90 \%$ at concentrations lower than $10 \mu \mathrm{M}$. Moreover, (79) 6-epiterminaloside K and (80) terminaloside C, (81) terminaloside $\mathrm{F}$, and (82) terminaloside I showed antiestrogenic activity against MCF-7 cells. The antiproliferative potential of these compounds was mainly attributed to the metabolism of the gut microbiota, which produces mammalian lignan metabolites with estrogenic activity like (83) enterodiol and (84) enterolactone (Muhit et al., 2016).

Also, leaf ethanol extracts of T. catappa L. from Taiwan was reported by Lee et al. (2019), who used HeLa and SiHa cervical cancer cell lines to test its antiproliferative effect. The authors evaluated Terminalia sp. extracts at a concentration of 25, 50, 75, and $100 \mu \mathrm{g} / \mathrm{mL}$. It was shown that Terminalia sp. extracts have low cytotoxicity and suppress TPA-induced migration and invasion through the inhibition of MMP-9 and ERK1/2 phosphorylation in the cell lines in a dose-dependent manner (Lee et al., 2019). Likewise, water extracts from T. chebula Retz. from the Republic of Korea reported by Lee et al. (2016), showed antiproliferative potential in HeLa cell lines. The authors state that $T$. chebula Retz. extracts antagonize with the production of mitochondrial-derived reactive oxygen species, which may be related to the author's hypothesis that due to the previously reported antioxidant activity of $T$. chebula Retz. This extract may have a role in the inhibition of TNF-induced necroptotic cell death. Moreover, HPLC and UHPLC-MS analyses exhibited that water extracts from $T$. chebula Retz. were rich in (3) gallic acid (553.79 nmol/mg), (85) geraniin ( $80.78 \mathrm{nmol} / \mathrm{mg}),(76)$ chebulic acid (54.60 nmol/mg), (86) punicalagin (10.48 nmol/mg), (9) chebulinic acid (10.32 nmol/mg), and (53) chebulagic acid (9.24 $\mathrm{nmol} / \mathrm{mg}$ ). These compounds may be related to its necroptotic cell death induction activity (Lee et al., 2016).

Another report attributes the antiproliferative activity of $T$. chebula Retz. to (53) chebulagic acid in a dose-dependent manner in retinoblastoma cells. The mode of action was suggested through modulation of MMP, induction of the release of cytochrome c, activated caspase 3, and modulated ratio of BAX and Bcl2 in cell death (Kumar et al., 2014). Kumar et al. (2014), treated Y79 cells with different (53) chebulagic acid concentrations $(0.001,0.01,0.1,0.5,1,5,10,25,50$, and $100 \mu \mathrm{M})$, which decreased the proliferation of the cells in a dosedependent manner. Only at a concentration of $50 \mu \mathrm{M}$ of (53) chebulagic acid, a 50\% antiproliferative effect was observed, which was attributed to the capacity of (53) chebulagic acid to induce G1 arrest, inhibit $\mathrm{NF \kappa B}$ and induce apoptosis in retinoblastoma Y79 cell lines by induction of the release of cytochrome $\mathrm{c}$ by modulating the mitochondrial membrane potential and altering $\mathrm{BAX} / \mathrm{Bcl} 2$ ratio. Also, Terminalia sp. extracts have shown antiproliferative potential against breast cancer, as it is reported by Ghate et al. (2014). The authors showed that methanolic extracts from T. bellerica (Gaertn.) Roxb. at a concentration of $100 \mu \mathrm{g} / \mathrm{mL}$ had antiproliferative activity against human breast MCF-7 and human lung A549 carcinoma cell lines. Terminalia sp. extracts induced apoptosis by affecting the Bax/Bcl-2 ratio (proapoptotic and antiapoptotic proteins, respectively) in both cell types. HPLC analysis of $T$.

TABLE 5 | Summarization of the antiinflammatory studies of different Terminalia species.

\begin{tabular}{|c|c|c|c|}
\hline Terminalia sp. & Mechanism/Mode of action & Phytochemicals (compound no.) & Reference \\
\hline $\begin{array}{l}\text { T. coriacea (Roxb.) Wight } \\
\text { \& Arn. }\end{array}$ & $\begin{array}{l}\text { Decreased paw volume and wet and dry weights } \\
\text { of granulomatous tissue in acute and chronic } \\
\text { models of inflammation in rats }\end{array}$ & $\begin{array}{l}\text { (97) Apigenin, (36) kaempferol, (48) luteolin, (98) myricetin, (35) } \\
\text { quercetin, and (118) rutin }\end{array}$ & (Khan et al., 2018) \\
\hline T. bellerica (Gaertn.) Roxb. & $\begin{array}{l}\text { Selective binding of gallic acid with the amino } \\
\text { acids Arg120 and Glu524 from COX-2 }\end{array}$ & (3) Gallic acid & (Reddy et al., 2010) \\
\hline $\begin{array}{l}\text { T. glaucescens Planch. ex } \\
\text { Benth. }\end{array}$ & Inhibition of albumin denaturation and hemolysis & $\begin{array}{l}\text { (99) termiglaucescin; (100) } \beta \text {-D-glucopyranosyl } 2 \alpha, 3 \beta, 6 \beta \text { - } \\
\text { trihydroxy-23-gallylean-12-en-28-oate; (101) arjunglucoside I; } \\
\text { (102) sericoside; (103) arjungenin; (104) sricic acid; (105) } \\
\text { arjunetin; (106) chebuloside II; (107) 3,3'4-tri-O-methylelagic acid; } \\
\text { (108) 3,3'-di-O-methylelagic acid; (37) } \beta \text {-sitosterol; and (10) } \\
\text { stigmasterol }\end{array}$ & (Dawe et al., 2017) \\
\hline T. muelleri Benth. & $\begin{array}{l}\text { Reduction of paw edema in carrageenan-induced } \\
\text { paw edema in mice. Reduced proinflammatory }\end{array}$ & $\begin{array}{l}\text { (109) luteolin-6-C-glucoside, (110) vitexin, (4) ellagic acid, and (9) } \\
\text { chebulinic acid }\end{array}$ & (Fahmy et al., 2017) \\
\hline
\end{tabular}

cytokines PGE2, TNF- $\alpha$, IL-1 $\beta$, and IL-6 
bellerica (Gaertn.) Roxb. fruits methanolic extracts exhibited (35) quercetin, (3) gallic acid, (87) catechin, (88) reserpine, and (89) tannic acid as potential active constituents. A Summary of the anticancer studies with different Terminalia species is presented in Table 4. In addition, Figure 4(ii) shows the structures of some of the active constituents with potential antiproliferative and anticancer properties of Terminalia species.

\section{Antiinflammatory Properties of Terminalia sp.}

Inflammation plays a key role in many human diseases. Recent studies show that many noncommunicable diseases share common pathophysiological mechanisms, where oxidative stress and inflammation play a major role in the onset and development of these diseases (Camps and García-Heredia, 2014). Inflammation has been related to obesity, diabetes, cancer, cardiovascular diseases, among others, through various mechanisms (Ghosh et al., 2015). Thus, many investigations have focused on the antiinflammatory pharmaceutical potential of phytochemicals and natural products, aiming to ameliorate adverse effects from antiinflammatory drugs (Ambriz-Pérez et al., 2016). In this sense, some Terminalia species have been reported with antiinflammatory activity in vitro, which is regularly attributed to their phytochemical composition; the association between the antiinflammatory activity and the structural characteristics of some phytochemicals has already been reported (Gautam and Jachak, 2009; Lago et al., 2014). In this section, we summarize some of the most recent reports on the antiinflammatory potential of Terminalia species.

Khan et al. (2018), evaluated the anti-inflammatory capacity of Terminalia coriacea (Roxb.) Wight \& Arn. (Terminalia coriacea Spreng.), in albino Wistar rats with an acute and chronic model, carrageenan-induced paw edema and cotton pellet-induced granuloma, respectively. T. corriacea (Roxb.) Wight \& Arn. was evaluated at different concentrations (125, 250 , and $500 \mathrm{mg} / \mathrm{kg}$ ) by oral administration of the leaf extract. All tested concentrations showed antiinflammatory activity at a dose-dependent manner, which the authors attribute to the antioxidant potential of Terminalia sp. flavonoids like (97) apigenin, (36) kaempferol, (48) luteolin, (98) myricetin, (35) quercetin, and (118) rutin; which was reflected as a decreased paw volume and wet and dry weights of granulomatous tissue in both models of inflammation (Khan et al., 2018).

Terminalia chebula Retz. fruit ethanolic extract was evaluated at concentrations of 50 to $500 \mathrm{mg} / \mathrm{kg}$, p.o. against carrageenaninduced inflammation in rats. In this study, Bag et al. (2013), report an increased inhibitory potential on carrageenan-induced lipid peroxidation in rat liver in a dose-dependent manner, with the highest inhibition (84.08\%) at $250 \mathrm{mg} / \mathrm{kg}$, p.o.). A study by Reddy et al. (2010), evaluated the COX-2 inhibitory capacity of isolated (3) gallic acid from the ethanolic extract of Terminalia bellirica (Gaertn.) Roxb. fruits obtained by RP-HPLC. The authors report that (3) gallic acid has a concentrationdependent inhibitory capacity of COX-1 and COX-2, with $\mathrm{IC}_{50}$ values of $1.5 \mu \mathrm{M}$ and $74 \mu \mathrm{M}$, respectively. Further biochemical tests showed competitive binding of (3) gallic acid for both COX1 and COX-2 concerning substrate, and a time-dependent inhibition for both molecular targets. It is also reported that (3) gallic acid, binding to COX-2 is mediated via the carboxylate moiety of (3) gallic acid with the amino acids Arg120 and Glu524 at the entrance of the active site.

Dawe et al. (2017), identified and isolated 11 triterpenes from ethyl acetate extracts of the root bark of Terminalia glaucescens Planch. ex Benth.; the compounds were identified as (99) termiglaucescin; (100) $\beta$-D-glucopyranosyl $2 \alpha, 3 \beta, 6 \beta$ trihydroxy-23-gallylean-12-en-28-oate; (101) arjunglucoside I; (102) sericoside; (103) arjungenin; (104) sericic acid; (105) arjunetin; (106) chebuloside II; (107) 3,3'4-tri-O-methylelagic acid; (108) 3,3'-di-O-methylelagic acid; (37) $\beta$-sitosterol; and (10) stigmasterol. Moreover, these compounds also showed anti-inflammatory activity by inhibition of albumin denaturation and hemolysis (Dawe et al., 2017). Furthermore, the anti-inflammatory activity of Terminalia muelleri Benth. polyphenol-rich extracts were determined by Fahmy et al. (2017), using carrageenan-induced paw edema model in mice by measuring the thickness of the injected paws after treatment with Terminalia sp. extract at a concentration of 100, 200, and $400 \mathrm{mg} / \mathrm{kg}$. Pretreatment with Terminalia sp. extracts exhibited a dose-dependent significant anti-inflammatory activity showed in the reduction in the carrageenan-induced paw edema by 48,53 ,

TABLE 6 | Summarization of the antioxidant studies of different Terminalia species.

\begin{tabular}{ll}
\hline Terminalia sp. & \multicolumn{1}{c}{ Mechanism/Mode of action } \\
\hline $\begin{array}{l}\text { T. sericea Burch. ex DC. } \\
\text { T. chebula Retz. }\end{array}$ & $\begin{array}{l}\text { Inhibition of the DPPH radical } \\
\text { Scavenging activity towards the DPPH radical, nitric } \\
\text { oxide and } \mathrm{H}_{2} \mathrm{O}_{2}\end{array}$ \\
$\begin{array}{l}\text { Scavenging activity against the DPPH radical } \\
\text { T. chebula Retz. }\end{array}$ & $\begin{array}{l}\text { Scavenging activity against the ABTS and DPPH } \\
\text { radicals }\end{array}$ \\
T. chebula Retz. & $\begin{array}{l}\text { Normalized levels of lipid peroxidation, nitric oxide, } \\
\text { superoxide dismutase, glutathione peroxidase, and } \\
\text { catalase in rats with acetic acid-induced colitis }\end{array}$ \\
T. chebula Retz. & $\begin{array}{l}\text { Optimized ultrasound-assisted extraction of ethanol } \\
\text { extracts yields compounds with DPPH and ABTS } \\
\text { scavenging activity }\end{array}$
\end{tabular}

Phytochemicals (compound no.)
(3) gallic acid and (113) resveratrol
Not identified
Not identified
(114) purpurin, (87) catechin, (89) tannic acid, (88)
reserpine, (4) ellagic acid, (70) methyl gallate, and (118)
rutin
Tannins, triterpenoids, phenolic compounds

(114) shikimic acid; (3) gallic acid; (115) 5-Ogalloylshikimic acid; (5) corilagin; (116) 3,4,8,9,10pentahydroxydibenzo (b,d) pyran-6-one; and (4) ellagic acid
Reference

(Anokwuru et al., 2018)

(Saha and Verma, 2016)

(Bag et al., 2013)

(Basu et al., 2017)

(Gautam et al., 2013)

(Sheng et al., 2018) scavenging activity 
and $62 \%$ at 100,200 , and $400 \mathrm{mg} / \mathrm{kg}$, respectively. Moreover, pretreatment with Terminalia sp. extracts also decreased in a dose-dependent manner, the pro-inflammatory cytokines PGE2, TNF- $\alpha$, IL-1 $\beta$, and IL-6. The authors suggested that this effect might be attributed to the presence of (109) luteolin-6-Cglucoside, (110) vitexin, (4) ellagic acid, and (9) chebulinic acid in the extracts (Fahmy et al., 2017).

Crude extracts from Terminalia phanerophlebia Engl. \& Diels, a species endemic to Africa, exhibit selective inhibition of COX-2 (92.4\%). The bioactivity was attributed to the cholestane triterpenoids such as (37) $\beta$-sitosterol, (111) $\beta$ sitostenone, and (112) stigmast-4-ene-3,6-dione; which were further isolated and individually evaluated, this exhibited that the COX-2 inhibitory activity might be attributed to the triterpenoid (37) $\beta$-sitosterol (Nair et al., 2012). Similarly, Terminalia bellirica (Gaertn.) Roxb. ethyl acetate from the aerial parts at concentrations of 100 and $300 \mathrm{mg} / \mathrm{kg}$ was orally administered to male $\mathrm{BALB} / \mathrm{cN}$ mice following tetrachlorideintoxication. Terminalia sp. treatment downregulated the expression of the inflammatory mediators NF- $\kappa \mathrm{B}, \mathrm{COX}-2$, and TNF- $\alpha$. A summary of the antiinflammatory studies of different Terminalia species is presented in Table 5. The chemical structures of some bioactive compounds from Terminalia species with antiinflammatory potential are shown in Figure 4(iii).

Antioxidant Potential of Terminalia sp.

Oxidative stress is frequently defined as the imbalance between antioxidants and oxidants in favor of the oxidants, and this may lead to oxidative damage to molecules of biological importance (Halliwell and Gutteridge, 2015). Oxidative stress main arises from diverse factors such as diminished levels of antioxidant enzymes, increased production of reactive species (due to unhealthy lifestyles, smoking, abusive intake of alcohol, among others). Oxidative stress has been

TABLE 7 | Antibacterial potential of Terminalia sp

Plant species/part Extraction procedure

Terminalia arjuna (Roxb. ex DC.) Wight \& Arn. bark

Terminalia arjuna (Roxb. ex DC.) Wight \& Arn. leaf

Terminalia bellirica (Gaertn.) Roxb. fruit

Terminalia catappa L. leaf

Terminalia chebula Retz. fruit

Terminalia ferdinandiana Exell bark

Terminalia ferdinandiana Exell fruit

Terminalia ferdinandiana Exell seed coat

Terminalia glaucescens Planch. ex Benth. root

Terminalia superba Engl. \& Diels bark

\begin{tabular}{|c|c|c|c|}
\hline Extraction procedure & Pathogenic bacteria & Antibacterial activity & Reference \\
\hline Ethanol extraction & B. cereus & $\mathrm{IZ}=7 \mathrm{~mm}$ & (Saivaraj and Chandramohan, \\
\hline Ethanol extraction & $P$. aeruginosa & $\mathrm{IZ}=8 \mathrm{~mm}$ & 2018) \\
\hline Ethanol extraction & S aureus & $\mathrm{MIC}=1.56 \mathrm{mg} / \mathrm{mL}$ & (Aneja et al., 2012) \\
\hline Ethanol extraction & E. coli & $\mathrm{MIC}=50 \mathrm{mg} / \mathrm{mL}$ & \\
\hline Ethanol extraction & P. mirabilis & $\mathrm{MIC}=50 \mathrm{mg} / \mathrm{mL}$ & \\
\hline Ethanol extraction & S. aureus & $\mathrm{MIC}=6.25 \mathrm{mg} / \mathrm{mL}$ & (Aneja et al., 2012) \\
\hline Ethanol extraction & $P$. aeruginosa & $\mathrm{MIC}=50 \mathrm{mg} / \mathrm{mL}$ & \\
\hline Ethanol extraction & P. mirabilis & $\mathrm{MIC}=6.25 \mathrm{mg} / \mathrm{mL}$ & \\
\hline $\begin{array}{l}\text { Direct aqueous extracts/Reflux } \\
\text { method }\end{array}$ & MRSA & $\mathrm{MIC}=0.25 \mathrm{mg} / \mathrm{mL}$ & (Dharmaratne et al., 2018) \\
\hline $\begin{array}{l}\text { Direct aqueous extracts/Reflux } \\
\text { method }\end{array}$ & MDR Acinetobacter sp. & $\mathrm{MIC}=0.5 \mathrm{mg} / \mathrm{mL}$ & \\
\hline $\begin{array}{l}\text { Direct aqueous extracts/Reflux } \\
\text { method }\end{array}$ & MDR $P$. aeruginosa & $\mathrm{MIC}=0.5 \mathrm{mg} / \mathrm{mL}$ & \\
\hline Ethanol extraction & B. subtilis & $\mathrm{MIC}=3 \mathrm{mg} / \mathrm{mL}$ & (Suparno et al., 2018) \\
\hline Ethanol extraction & E. coli & $\mathrm{MIC}=3 \mathrm{mg} / \mathrm{mL}$ & \\
\hline Ethanol extraction & $P$. aeruginosa & $\mathrm{IZ}=1.83-6.5 \mathrm{~mm}$ & (Allyn et al., 2018) \\
\hline Ethanol extraction & S. aureus & $\mathrm{IZ}=1.73-9.06 \mathrm{~mm}$ & \\
\hline Ethanol extraction & Acinetobacter sp. & $\mathrm{MIC}=12.5 \mathrm{mg} / \mathrm{mL}$ & (Sharma et al., 2012) \\
\hline Ethanol extraction & E. coli & $\mathrm{MIC}=50 \mathrm{mg} / \mathrm{mL}$ & \\
\hline Ethanol extraction & P. mirabilis & $\mathrm{MIC}=25 \mathrm{mg} / \mathrm{mL}$ & \\
\hline Ethanol extraction & P. aeruginosa & $\mathrm{MIC}=12.5 \mathrm{mg} / \mathrm{mL}$ & \\
\hline Ethanol extraction & S. aureus & $\mathrm{MIC}=3.12 \mathrm{mg} / \mathrm{mL}$ & \\
\hline Ethanol extraction & B. cereus & $\mathrm{IZ}=13.2 \mathrm{~mm}$ & (Akter et al., 2019) \\
\hline Ethanol extraction & MRSA & $\mathrm{IZ}=12.7 \mathrm{~mm}$ & \\
\hline Ethanol extraction & B. cereus & $\mathrm{IZ}=17.8 \mathrm{~mm}$ & \\
\hline Ethanol extraction & L. monocytogenes & $\mathrm{IZ}=18.5 \mathrm{~mm}$ & \\
\hline Ethanol extraction & MRSA & $\mathrm{IZ}=17.1 \mathrm{~mm}$ & \\
\hline Ethanol extraction & B. cereus & $\mathrm{IZ}=9.8 \mathrm{~mm}$ & \\
\hline Ethanol extraction & L. monocytogenes & $\mathrm{IZ}=10.8 \mathrm{~mm}$ & \\
\hline Methanol extraction & MRSA & $\mathrm{IZ}=8.8 \mathrm{~mm}$ & \\
\hline Ethanol extraction & E. aerogenes & $\mathrm{MIC}=100 \mathrm{mg} / \mathrm{mL}$ & (Gbala and Anibijuwon, 2018) \\
\hline Ethanol extraction & K. pneumoniae & $\mathrm{MIC}=0.1 \mathrm{mg} / \mathrm{mL}$ & \\
\hline Ethanol extraction & P. mirabilis & $\mathrm{MIC}=100 \mathrm{mg} / \mathrm{mL}$ & \\
\hline Methanol extraction & E. coli & $\mathrm{MIC}=0.078 \mathrm{mg} / \mathrm{mL}$ & (Kuete et al., 2010) \\
\hline Methanol extraction & K. pneumoniae & $\mathrm{MIC}=0.078 \mathrm{mg} / \mathrm{mL}$ & \\
\hline Methanol extraction & M. tuberculosis & $\mathrm{MIC}=0.078 \mathrm{mg} / \mathrm{mL}$ & \\
\hline Methanol extraction & P. aeruginosa & $\mathrm{MIC}=0.019 \mathrm{mg} / \mathrm{mL}$ & \\
\hline Methanol extraction & S. dysenteriae & $\mathrm{MIC}=0.039 \mathrm{mg} / \mathrm{mL}$ & \\
\hline Methanol extraction & S. typhi & $\mathrm{MIC}=0.078 \mathrm{mg} / \mathrm{mL}$ & \\
\hline Ethanol extraction & S. aureus & $\mathrm{MIC}=0.078 \mathrm{mg} / \mathrm{mL}$ & (Elvire et al., 2018) \\
\hline
\end{tabular}

IZ, inhibition zone. 
TABLE 8 | Antifungal activity of Terminalia sp. against pathogenic fungi.

\begin{tabular}{|c|c|c|c|c|}
\hline Plant species/part & Extraction Procedure & $\begin{array}{l}\text { Pathogenic } \\
\text { fungi }\end{array}$ & Antifungal activity & Reference \\
\hline \multirow[t]{5}{*}{$\begin{array}{l}\text { Terminalia arjuna (Roxb. ex DC.) Wight \& Arn. } \\
\text { bark }\end{array}$} & Ethanol extraction & A. flavus & $\mathrm{IZ}=7 \mathrm{~mm}$ & $\begin{array}{l}\text { (Saivaraj and Chandramohan, } \\
\text { 2018) }\end{array}$ \\
\hline & Ethanol extraction & C. albicans & $\mathrm{IZ}=15 \mathrm{~mm}$ & (Debnath et al., 2013) \\
\hline & Ethanol extraction & C. glabrata & $\mathrm{IZ}=20 \mathrm{~mm}$ & \\
\hline & Ethanol extraction & C. krusei & $\mathrm{IZ}=20 \mathrm{~mm}$ & \\
\hline & Ethanol extraction & C. tropicalis & $\mathrm{IZ}=18 \mathrm{~mm}$ & \\
\hline Terminalia catappa L. bark & $\begin{array}{l}\text { Ethanol extraction/Methanol } \\
\text { extraction }\end{array}$ & F. oxysporum & $\mathrm{MIC}=0.025 \mathrm{mg} / \mathrm{mL}$ & (Kouassi et al., 2019) \\
\hline \multirow[t]{4}{*}{ Terminalia catappa L. leaf } & Ethanol extraction & C. albicans & $\begin{array}{l}\mathrm{MIC}=0.25 \mathrm{mg} / \mathrm{mL} \\
\text { Biofilm inhibition }\end{array}$ & (Gonçalves et al., 2019) \\
\hline & Ethanol extraction & C. glabrata & $\begin{array}{l}\mathrm{MIC}=0.25 \mathrm{mg} / \mathrm{mL} \\
\text { Biofilm inhibition }\end{array}$ & \\
\hline & Ethanol extraction & C. krusei & $\mathrm{MIC}=1.5 \mathrm{mg} / \mathrm{mL}$ & \\
\hline & Ethanol extraction & C. tropicalis & $\mathrm{MIC}=1.5 \mathrm{mg} / \mathrm{mL}$ & \\
\hline \multirow[t]{3}{*}{ Terminalia chebula Retz. fruit } & Ethanol extraction & C. albicans & $\mathrm{MIC}=0.25 \mathrm{mg} / \mathrm{mL}$ & (Vidya et al., 2019) \\
\hline & Ethanol extraction & C. glabrata & $\mathrm{MIC}=0.25 \mathrm{mg} / \mathrm{mL}$ & \\
\hline & Ethanol extraction & C. tropicalis & $\mathrm{MIC}=0.050 \mathrm{mg} / \mathrm{mL}$ & \\
\hline \multirow[t]{3}{*}{ Terminalia glaucescens Planch. ex Benth. stem } & Ethanol extraction & A. flavus & $\mathrm{IZ}=12.0 \mathrm{~mm}$ & (Adeeyo et al., 2018) \\
\hline & Ethanol extraction & A. niger & $\mathrm{IZ}=11.0 \mathrm{~mm}$ & \\
\hline & Ethanol extraction & Candida sp. & $\mathrm{IZ}=11.0 \mathrm{~mm}$ & \\
\hline \multirow[t]{2}{*}{ Terminalia ivorensis A.Chev. bark } & Ethanol extraction & F. oxysporum & $\mathrm{MIC}=0.050 \mathrm{mg} / \mathrm{mL}$ & (Kouassi et al., 2019) \\
\hline & Ethanol extraction & F. graminearum & $\mathrm{MIC}=0.025 \mathrm{mg} / \mathrm{mL}$ & \\
\hline \multirow[t]{2}{*}{ Terminalia mantaly H. Perrier bark } & Ethanol extraction & F. oxysporum & $\mathrm{MIC}=0.050 \mathrm{mg} / \mathrm{mL}$ & (Kouassi et al., 2019) \\
\hline & Ethanol extraction & F. graminearum & $\mathrm{MIC}=0.025 \mathrm{mg} / \mathrm{mL}$ & \\
\hline \multirow[t]{6}{*}{ Terminalia superba Engl. \& Diels bark } & Methanol extraction & C. albicans & $\mathrm{MIC}=0.039 \mathrm{mg} / \mathrm{mL}$ & (Kuete et al., 2010) \\
\hline & Methanol extraction & C. glabrata & $\mathrm{MIC}=0.078 \mathrm{mg} / \mathrm{mL}$ & \\
\hline & Methanol extraction & M. audouinii & $\mathrm{MIC}=0.019 \mathrm{mg} / \mathrm{mL}$ & \\
\hline & Methanol extraction & T. rubrum & $\mathrm{MIC}=0.039 \mathrm{mg} / \mathrm{mL}$ & \\
\hline & Ethanol extraction & F. oxysporum & $\mathrm{MIC}=0.050 \mathrm{mg} / \mathrm{mL}$ & (Kouassi et al., 2019) \\
\hline & Ethanol extraction & F. graminearum & $\mathrm{MIC}=0.050 \mathrm{mg} / \mathrm{mL}$ & \\
\hline
\end{tabular}

IZ, inhibition zone.

related to the onset of many diseases and their comorbidities (Camps and García-Heredia, 2014; Halliwell and Gutteridge, 2015). Terminalia species are a rich source of phytochemicals such as terpenes, flavonoids, and phenolic acids, molecules with reported antioxidant activity. This section summarizes some of the most recent publications on this subject.

Anokwuru et al. (2018), evaluated the antioxidant capacity of ethanolic extracts of fruit, leaf, stem, bark, and roots of Terminalia sericea Burch. ex DC. from South Africa; moreover, free, conjugated, and bound phenolic-rich extracts were obtained. The antioxidant activity was evaluated by the DPPH method. The authors reported that the free fruit extracts, ester bound of leaves and roots, glycoside bound fruit extracts, leaves, and stem insoluble bound extracts showed the highest antioxidant activity with $\mathrm{IC}_{50}$ values of $3.13,4.58$, and 4.89 , 12.6, 15.4, and $17.8 \mu \mathrm{g} / \mathrm{mL}$, respectively. Furthermore, the antioxidant activity was attributed mainly to the presence of (3) gallic acid and (113) resveratrol. Another evaluation of polyphenolic-rich extracts was performed by Saha and Verma (2016), but with Terminalia chebula Retz. fruit methanolic extracts at concentrations ranging from $50-500 \mu \mathrm{g} / \mathrm{mL}$; the authors evaluated the antioxidant capacity of the samples by different methods. The total antioxidant capacity assay showed that $T$. chebula Retz. extracts had the highest antioxidant potential, in a dose-dependent manner, at a concentration of $150 \mu \mathrm{g} / \mathrm{mL}$ yielding an $\mathrm{IC}_{50}$ value of $14 \mu \mathrm{g} / \mathrm{mL}$. Moreover, $T$. chebula Retz. extracts also inhibited nitric oxide with an $\mathrm{IC}_{50}$ value of $30.51 \mu \mathrm{g} / \mathrm{mL}$ at a concentration of $500 \mu \mathrm{g} / \mathrm{mL}$; the extracts also showed scavenging activity towards $\mathrm{H}_{2} \mathrm{O}_{2}$, which is not a free radical but the precursor of some reactive oxygen species, with an $\mathrm{IC}_{50}$ value of $265.53 \mu \mathrm{g} / \mathrm{mL}$.

Studies on Terminalia chebula Retz. collected in India and extracts prepared from fruits with $70 \%$ ethanol were reported by Bag et al. (2013). The antioxidant capacity was measured by the liver lipid peroxidation and DPPH assays; T. chebula Retz. extracts exhibited a dose-dependent antioxidant capacity with the highest inhibition of the DPPH radical at a concentration of $50 \mu \mathrm{g} / \mathrm{mL}$ with $58.40 \%$ antioxidant percentage and $\mathrm{IC}_{50}$ of 42.14 $\mu \mathrm{g} / \mathrm{mL}$. Moreover, the extracts also reduced the formation of MDA in a dose-dependent manner, with the highest activity at a dose of $250 \mathrm{mg} / \mathrm{kg}$ p.o.

Methanolic extracts of fruits of T. bellerica (Gaertn.) Roxb. from India were studied by Basu et al. (2017). The extracts were further sequentially extracted with n-hexane, chloroform, ethyl acetate, butanol, and water to obtain different fractions. The obtained extracts were evaluated by the Trolox Equivalent Antioxidant Capacity method, and the results showed that the butanol, water, and ethyl acetate extracts had the highest antioxidant capacity. Moreover, the ethyl acetate extracts showed the highest DPPH scavenging radical activity with an $\mathrm{IC}_{50}$ value of $7.11 \mu \mathrm{g} / \mathrm{mL}$. The authors mention that the polar fractions showed higher antioxidant activity might be related to 
TABLE 9 | Antiparasitic activity of Terminalia sp. against pathogenic parasites.

\begin{tabular}{|c|c|c|c|c|}
\hline Plant species/part & Extraction Procedure & $\begin{array}{l}\text { Pathogenic } \\
\text { parasites }\end{array}$ & Antiparasitic activity & Reference \\
\hline Terminalia albida Scott-Elliot bark & $\begin{array}{l}\text { Methanol extraction } \\
\text { Methanol extraction }\end{array}$ & $\begin{array}{l}\text { P. falciparum } \\
\text { P. berghei }\end{array}$ & $\begin{array}{l}\mathrm{IC}_{50}=1.5 \mu \mathrm{g} / \mathrm{mL} \\
\text { In vivo, Terminalia albida Scott-Elliot } \\
\text { treatment } \\
\text { increased survival rates in P. berghei- } \\
\text { infected mice. }\end{array}$ & (Camara et al., 2019) \\
\hline \multirow[t]{2}{*}{$\begin{array}{l}\text { Terminalia arjuna (Roxb. ex DC.) Wight } \\
\& \text { Arn. bark }\end{array}$} & $\begin{array}{l}\text { Methanol in a Soxhlet's } \\
\text { apparatus }\end{array}$ & H. contortus ova & $\mathrm{IC}_{50}=645.65 \mu \mathrm{g} / \mathrm{mL}$ & (Bachaya et al., 2009) \\
\hline & $\begin{array}{l}\text { Methanol in a Soxhlet's } \\
\text { apparatus }\end{array}$ & H. contortus larva & $\mathrm{IC}_{50}=467.74 \mu \mathrm{g} / \mathrm{mL}$ & \\
\hline $\begin{array}{l}\text { Terminalia avicennioides Guill. \& Perr. } \\
\text { bark }\end{array}$ & Powder form & P. berghei & Inhibition of the malaria in infected mice & (Owoloye et al., 2019) \\
\hline Terminalia catappa L. fruit & Ethanol extraction & P. posthuman & At $60 \mathrm{mg} / \mathrm{ml}$; death time $=28 \mathrm{~min}$ & (Ingole et al., 2019) \\
\hline \multirow[t]{4}{*}{ Terminalia catappa L. leaf } & Ethanol extraction & F. cobboldi & Motility inhibition within $3 \mathrm{~h}$ & (Anuracpreeda et al., 2016) \\
\hline & Butanol extraction & C. spatiosus & $\mathrm{LC}_{50}=487.17 \mathrm{ppm}$ & (Minsakorn et al., 2019) \\
\hline & Ethyl acetate extraction & P. falciparum & $\mathrm{IC}_{50}=3.05 \mu \mathrm{g} / \mathrm{mL}$ & (Abiodun et al., 2011) \\
\hline & Ethyl acetate extraction & T. brucei rhodesiense & $\mathrm{IC}_{50}=7.80 \mu \mathrm{g} / \mathrm{mL}$ & (Abiodun et al., 2012) \\
\hline Terminalia ferdinandiana Exell fruit & Aqueous extraction & G. duodenalis & $\mathrm{IC}_{50}=140 \mu \mathrm{g} / \mathrm{mL}$ & (Rayan et al., 2015) \\
\hline Terminalia ferdinandiana Exell fruit pulp & - & G. duodenalis & $\mathrm{LC}_{50}=1,150 \mu \mathrm{g} / \mathrm{mL}$ & (Cock and Rayan, 2020) \\
\hline Terminalia mantaly $\mathrm{H}$. Perrier leaf & Aqueous extraction & P. falciparum & $\mathrm{IC}_{50}=2.09 \mu \mathrm{g} / \mathrm{mL}$ & (Mbouna et al., 2018) \\
\hline \multirow[t]{3}{*}{ Terminalia mantaly H. Perrier root } & Methanol extraction & P. falciparum & $\mathrm{IC}_{50}=7.01 \mu \mathrm{g} / \mathrm{mL}$ & (Mbouna et al., 2018) \\
\hline & Methanol extraction & P. falciparum & $\mathrm{IC}_{50}=10.11 \mu \mathrm{g} / \mathrm{mL}$ & (Mbosso Teinkela et al., 2019) \\
\hline & Hexane fraction & $\begin{array}{l}\text { Trypanosoma brucei } \\
\text { brucei }\end{array}$ & $\mathrm{IC}_{50}=5.60 \mu \mathrm{g} / \mathrm{mL}$ & \\
\hline Terminalia mantaly H. Perrier stem & Methanol extraction & P. falciparum & $\mathrm{IC}_{50}=3.63 \mu \mathrm{g} / \mathrm{mL}$ & (Mbouna et al., 2018) \\
\hline Terminalia paniculata Roth root & $\begin{array}{l}\text { Ethanol in a Soxhlet's } \\
\text { apparatus }\end{array}$ & P. posthuma & $25 \mathrm{mg} / \mathrm{ml}$; death time $=87 \mathrm{~min}$ & $\begin{array}{l}\text { (Acharyya and Prasenjit } \\
\text { Bhuniya, 2019) }\end{array}$ \\
\hline \multirow[t]{2}{*}{ Terminalia superba Engl. \& Diels leaf } & $\begin{array}{l}\text { Methanol in a Soxhlet's } \\
\text { apparatus }\end{array}$ & $\begin{array}{l}\text { Trypanosoma brucei } \\
\text { brucei }\end{array}$ & $\mathrm{MIC}=1.6 \mathrm{mg} / \mathrm{mL}$ & (Antia et al., 2009) \\
\hline & Methanol extraction & P. falciparum & $\mathrm{IC}_{50}=3.38 \mu \mathrm{g} / \mathrm{mL}$ & (Mbouna et al., 2018) \\
\hline
\end{tabular}

the presence of compounds like (114) purpurin, (87) catechin, (89) tannic acid, (88) reserpine, (4) ellagic acid, (70) methyl gallate, and (118) rutin (Basu et al., 2017). Furthermore, Dawe et al. (2017), showed that ethanolic extracts from roots of $T$. glaucescens Planch. ex Benth. have scavenging activity against the $\mathrm{DPPH}$ radical, which may be attributed to the compounds (99) termiglaucescin; (100) $\beta$-D-glucopyranosyl $2 \alpha, 3 \beta, 6 \beta$ trihydroxy-23-gallylean-12-en-28-oate; (101) arjunglucoside I; (102) sericoside; (103) arjungenin; (104) sericic acid; (105) arjunetin; (106) chebuloside II; (107) 3,3'4-tri-O-methylelagic acid; (108) 3,3'-di-O-methylelagic acid; (37) $\beta$-sitosterol; and (10) stigmasterol (Dawe et al., 2017).

Terminalia chebula Retz. fruit extracts were tested for their antioxidant and antiinflammatory properties on acetic acidinduced colitis in inbred Charles-Foster strain albino rats and mice by Gautam et al. (2013). This report states that T. chebula Retz. extracts reversed the decreased levels of superoxide dismutase, catalase, and glutathione peroxidase caused by acetic acid-induced colitis, Terminalia sp. treatment also decreased the levels of lipid peroxidation and nitric oxide to normal levels, from 11 to 6.22 , and 10 to $4.25 \mathrm{nmol} / \mathrm{mg}$ protein, respectively. A preliminary phytochemical screening test showed that the main constituents in $T$. chebula Retz. extracts are tannins, phenolic compounds, and triterpenoids; however, further characterization is needed (Gautam et al., 2013).

Furthermore, Rashed et al. (2014), evaluated the antioxidant capacity of extracts from the aerial parts of T. bellerica (Gaertn.)
Roxb. on carbon tetrachloride-intoxicated mice. Preliminary antioxidant assays by the DPPH and ABTS tests showed that ethanolic extracts had a higher antioxidant activity with values of 2883.38 and $2414.81 \mathrm{TEAC} / \mathrm{mg}$ against the radicals, respectively. Moreover, in vivo studies showed that T. bellerica (Gaertn.) Roxb. extracts at a concentration of $300 \mathrm{mg} / \mathrm{kg}$ ameliorated in a dose-dependent manner, the production of 4-hydroxynonenal and 3-nitrotyrosine (Rashed et al., 2014). Terminalia chebula Retz. plant extracts obtained by ultrasonic-assisted extraction optimized using response surface methodology were reported by Sheng et al. (2018). The authors report the optimal factors for phenolic extraction of $T$. chebula Retz. were $68 \%$ ethanol concentration, the ultrasonic intensity of $3.6 \mathrm{~W} / \mathrm{cm}^{2}$, the particle size of $0.18 \mathrm{~mm}$, extraction temperature and time of $20 \mathrm{~min}$ for 2 times at $70^{\circ} \mathrm{C}$, and the liquid-solid ratio of $23 \mathrm{mg} /$ mL. Optimized results yield higher DPPH and ABTS antioxidant capacity in a dose-dependent manner, ultrasound-assisted extracts showed higher antioxidant activity than ascorbic acid by the FRAP method at $0.010-0.013 \mathrm{mg} / \mathrm{mL}$, and by the $\mathrm{DPPH}$ method at concentrations from $0.003-0.011 \mathrm{mg} / \mathrm{mL}$. An HPLCDAD-ESI-MS analysis showed the presence of (114) shikimic acid; (3) gallic acid; (115) 5-O-galloylshikimic acid; (5) corilagin; (116) 3,4,8,9,10-pentahydroxydibenzo (b,d) pyran-6-one; and (4) ellagic acid, could be responsible for the antioxidant properties of T. chebula Retz. fruit extracts (Sheng et al., 2018). A summary of the antioxidant potential of different Terminalia species is shown in Table 6 . The chemical structures of some 
TABLE 10 | Bio-activity of nanoparticles synthesized using Terminalia sp. extracts.

\begin{tabular}{|c|c|c|c|}
\hline Plant species/Part used & Plant-nanoparticle & Bio-activity & References \\
\hline $\begin{array}{l}\text { Terminalia arjuna (Roxb. ex } \\
\text { DC.) Wight \& Arn. bark }\end{array}$ & $\begin{array}{l}\text { Silver nanoparticles } \\
\text { (TA-AgNPs) }\end{array}$ & $\begin{array}{l}\text { TA-AgNPs inhibited E. coli, while the extract exhibited non-effects on the } \\
\text { organism. }\end{array}$ & (Ahmed et al., 2017) \\
\hline $\begin{array}{l}\text { Terminalia arjuna (Roxb. ex } \\
\text { DC.) Wight \& Arn. leaf }\end{array}$ & $\begin{array}{l}\text { Gold nanoparticles } \\
\text { (TA-AuNPs) }\end{array}$ & $\begin{array}{l}\text { TA-AuNPs induces the mitotic cell division and pollen germination. TA- } \\
\text { AuNPs showed a non-cytotoxic effect on Allium cepa root tip cells and } \\
\text { Gloriosa superba pollen grains. }\end{array}$ & (Gopinath et al., 2013) \\
\hline $\begin{array}{l}\text { Terminalia bellirica (Gaertn.) } \\
\text { Roxb. fruit pericarp }\end{array}$ & $\begin{array}{l}\text { Silver nanoparticles } \\
\text { (TB-AgNPs) }\end{array}$ & $\begin{array}{l}\text { TB-AgNPs at } 120 \mu \mathrm{g} / \mathrm{mL} \text { killed } 69.1 \% \text { colon cancer cells and } 65.2 \% \\
\text { breast cancer cells. The TB-AgNPs did not cause cytotoxic effects } \\
\text { against normal cardiac and skeletal muscle cells. }\end{array}$ & $\begin{array}{l}\text { (Nampoothiri et al., } \\
\text { 2018) }\end{array}$ \\
\hline $\begin{array}{l}\text { Terminalia belerica (Gaertn.) } \\
\text { Roxb. fruit }\end{array}$ & $\begin{array}{l}\text { Copper nanoparticles } \\
\text { (TM-CuONPs), } \\
\text { Iron nanoparticles } \\
\text { (TM-FeONPs), } \\
\text { Zinc nanoparticles } \\
\text { (TM-ZnONPs). }\end{array}$ & $\begin{array}{l}\text { Inhibition zone of TM-CuONPs, TM-FeONPs, and TM-ZnONPs against } \\
\text { Staphylococcus aureus ranged from 22-24 mM. }\end{array}$ & (Akhter et al., 2019) \\
\hline Terminalia catappa L. leaf & $\begin{array}{l}\text { Silver nanoparticles } \\
\text { (TC-AgNPs) }\end{array}$ & $\begin{array}{l}\text { TC-AgNPs showed antibacterial activity against both S. aureus and E. } \\
\text { coli. Nylon cloth-TC-AgNPs exhibited antibacterial activity against the } \\
\text { organisms with a strength of } 3 \text { to } 13 \text { times greater than TC-AgNPs. }\end{array}$ & $\begin{array}{l}\text { (Rohaeti and } \\
\text { Rakhmawati, 2017) }\end{array}$ \\
\hline Terminalia chebula Retz. leaf & Gold nanoparticles (TC-AuNPs) & $\begin{array}{l}\text { The antibacterial activity of TC-AuNPs against Gram-positive S. aureus } \\
\text { was better than Gram-negative E. coli measured by the well diffusion } \\
\text { method. }\end{array}$ & $\begin{array}{l}\text { (Mohan Kumar et al., } \\
\text { 2012) }\end{array}$ \\
\hline Terminalia mantaly H. Perrier & $\begin{array}{l}\text { Silver nanoparticles } \\
\text { (TM-AgNPs) }\end{array}$ & $\begin{array}{l}\text { MIC values of the TM-AgNPs }(3.12 \mu \mathrm{g} / \mathrm{mL}) \text { were } 40 \text { times lower than } \\
\text { those of the extract }(125 \mu \mathrm{g} / \mathrm{mL}) \text {. }\end{array}$ & $\begin{array}{l}\text { (Majoumouo et al., } \\
\text { 2019) }\end{array}$ \\
\hline $\begin{array}{l}\text { Terminali arjuna (Roxb. ex } \\
\text { DC.) Wight \& Arn. bark } \\
\text { extract }\end{array}$ & $\begin{array}{l}\text { Gold nanoparticles } \\
\text { (AuNPs) }\end{array}$ & $\begin{array}{l}\text { Co-administration with green synthesized AuNPs with size ranging } \\
\text { between } 20 \text { and } 40 \mathrm{nM} \text { along with acetaminophen showed effective } \\
\text { significant recovery in the hematological alterations of male Wistar rats. }\end{array}$ & (Mitra et al., 2019) \\
\hline $\begin{array}{l}\text { Terminalia arjuna (Roxb. ex } \\
\text { DC.) Wight \& Arn. bark } \\
\text { extract }\end{array}$ & $\begin{array}{l}\text { Gold nanoparticles } \\
\text { (TA-PdNPS) }\end{array}$ & $\begin{array}{l}\text { The TA-PdNPs were utilized as an efficient catalyst for Heck and Suzuki } \\
\text { type C-C coupling reactions and degradation of organic dyes in aqueous } \\
\text { medium making it useful in synthetic organic chemistry and the removal } \\
\text { of toxic industrial pollutants, respectively. }\end{array}$ & (Garai et al., 2018) \\
\hline $\begin{array}{l}\text { Terminalia arjuna (Roxb. ex } \\
\text { DC.) Wight \& Arn. bark } \\
\text { extract }\end{array}$ & $\begin{array}{l}\text { Silver nanoparticles } \\
\text { (AgNPs) }\end{array}$ & $\begin{array}{l}\text { AgNPs were spherical in shape ranges with } 40-50 \mathrm{nM} \text { in size. These } \\
\text { nanoparticles showed the inhibition of Staphylococcus aureus and } \\
\text { Pseudomonas aeruginosa bacteria. }\end{array}$ & $\begin{array}{l}\text { (Koparde and } \\
\text { Gaikwad, 2007) }\end{array}$ \\
\hline $\begin{array}{l}\text { Terminalia bellirica (Gaertn.) } \\
\text { Roxb. fruit extract }\end{array}$ & $\begin{array}{l}\text { Gold nanoparticles } \\
\text { (AuNPs) }\end{array}$ & $\begin{array}{l}\text { AuNPs were spherical shape ranges with } 20-30 \mathrm{nM} \text { and found to be } \\
\text { effective against Candida tropicalis and Candida albicans isolated from } \\
\text { clinical samples. AuNPs also effectively worked as free radical scavenging } \\
\text { activity. }\end{array}$ & $\begin{array}{l}\text { (Annavaram et al., } \\
\text { 2017) }\end{array}$ \\
\hline $\begin{array}{l}\text { Termanilia arjuna (Roxb. ex } \\
\text { DC.) Wight \& Arn. bark } \\
\text { extract }\end{array}$ & $\begin{array}{l}\text { Metal oxide nanoparticles: Copper } \\
\text { nanoparticles (CuNPs) and Zinc } \\
\text { nanoparticles (ZnNPs) }\end{array}$ & $\begin{array}{l}\text { CuNPs exhibited maximum antibacterial efficacy than ZnNPs against the } \\
\text { entire organism tested. K. pneumoniae showed high resistance to both } \\
\text { the biosynthesized nanoparticles. CuNPs exhibited maximum efficacy } \\
\text { when compared to ZNPs in antihemolytic activity against hypotonic and } \\
\text { heat-induced hemolysis of erythrocytes. }\end{array}$ & (Anuradha et al., 2017) \\
\hline $\begin{array}{l}\text { Terminalia arjuna (Roxb. ex } \\
\text { DC.) Wight \& Arn. leaf } \\
\text { extracts }\end{array}$ & $\begin{array}{l}\text { Gold nanoparticles } \\
\text { (AuNPs) }\end{array}$ & $\begin{array}{l}\text { AuNPs were treated with two different concentrations (500 and } \\
1,000 \mu \mathrm{M} \text { ) of Gloriosa superba seeds. Au NPs exposure at 1,000 } \mu \mathrm{M} \\
\text { concentration has the most significant effect on seed germination rate } \\
\text { and vegetative growth of G. superba. This is the first report on Au NPs } \\
\text { as a biocompatibility material to enhance the seed yield of this } \\
\text { endangered medicinal plant. }\end{array}$ & (Gopinath et al., 2013) \\
\hline $\begin{array}{l}\text { Terminalia arjuna (Roxb. ex } \\
\text { DC.) Wight \& Arn. bark } \\
\text { extract }\end{array}$ & $\begin{array}{l}\text { Copper nanoparticles } \\
\text { (CuNPs) }\end{array}$ & $\begin{array}{l}\text { The in vitro antimicrobial activity was found to be effective for CuNPs } \\
\text { dried at room temperature when compared to CuNPs dried at } 70^{\circ} \mathrm{C} \text {. } \\
\text { From this study, CuNPs shows a very good antioxidant property. }\end{array}$ & (Yallappa et al., 2013) \\
\hline $\begin{array}{l}\text { Terminalia catappa L. leaf } \\
\text { extract }\end{array}$ & $\begin{array}{l}\text { Gold nanoparticles } \\
\text { (AuNPs) }\end{array}$ & $\begin{array}{l}\text { Terminalia catappa L. (TC) leaf extract was treated with chloroauric acid } \\
\text { solutions, showing a rapid reduction of chloroaurate ions leading to the } \\
\text { formation of highly stable AuNPs in solution. AuNPs (10-35 nM size; } \\
\text { average size } 21.9 \mathrm{nM} \text { ) can be used as the reducing and stabilizing agent. }\end{array}$ & (Ankamwar, 2010) \\
\hline
\end{tabular}

bioactive compounds from Terminalia species with antioxidant potential is shown in Figure 4(iv).

\section{Antimicrobial Properties of Terminalia sp.} Terminalia species are documented with ethnobotanical used against infectious diseases/ailments such as conjunctivitis, diarrhea, dysentery, pneumonia, flu, and sore throats, sexually transmitted diseases, urinary infections, among others (Eloff et al.,
2008; Maulik and Katiyar, 2010; Maulik and Talwar, 2012; Cock, 2015; Afshari et al., 2016). This has led to some studies as an effort to elucidate their antimicrobial mechanisms and antimicrobial spectrum. Some efforts are being made to evaluate the potential antimicrobial properties of Terminalia sp. extracts. The report by Akter et al. (2019), evaluates the antimicrobial capacity of Terminalia ferdinandiana Exell. extracts in food preservation. The authors prepared the extracts with methanol, ethanol, 


\section{Terminalia chebula}

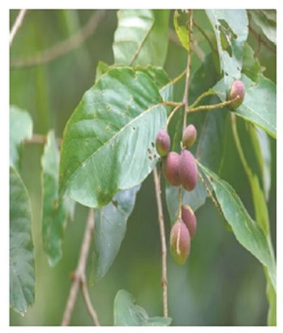

Anti-Alzheimer's mechanisms

\begin{tabular}{|c|}
\hline Anticholinesterase \\
$\qquad$\begin{tabular}{l} 
Gallotannin \\
Tannic acid \\
Gallic acid \\
Ellagic acid \\
\hline
\end{tabular}
\end{tabular}
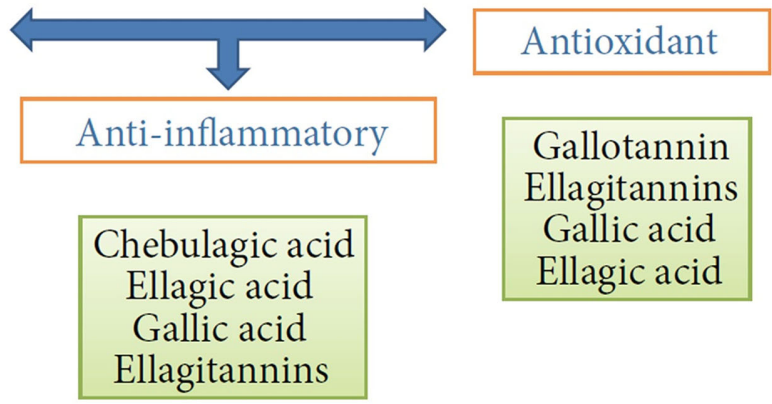

FIGURE 5 | Potential mechanism of anti Alzheimer's therapy through the anticholinesterase, antiinflammatory, and antioxidant properties of T. chebula Retz. Reproduced under the Creative Commons Attribution License (Afshari et al., 2016).

acetone, hexane, and distilled water, by accelerated solvent extraction from freeze-dried powders of barks, fruits, and leaves of T. ferdinandiana Exell, and tested them against some of the most common foodborne microorganisms by the disc diffusion assay. The authors found that methanol extracts showed a broad spectrum of antibacterial activity against the gram-positive Staphylococcus aureus, methicillin-resistant Staphylococcus aureus, Bacillus cereus, Listeria monocytogenes, and the gramnegative bacteria Pseudomonas aeruginosa. Also, the authors determined that the minimum inhibitory concentration and minimum bactericidal concentration values of extracts of $T$. ferdinandiana Exell ranged from 1-3 mg/mL, L. monocytogenes, $B$. cereus, and methicillin-resistant $S$. aureus were the most sensitive bacteria against Terminalia sp. extracts; on this subject, ethanol and acetone extracts showed the most potent antibacterial inhibitory activity. The authors argue that intriguingly, the extracts with the highest antioxidant activity (methanol and water extracts) were not the ones with the highest antibacterial activity, which may indicate that the compounds that may be differentially found in each type of extracts (Akter et al., 2019).

Moreover, T. bellirica (Gaertn.) Roxb. dried fruits were used to obtain direct and sequential dichloromethane, methanol, and water extracts, and to evaluate their antibacterial activity against 16 strains of methicillin-resistant Staphylococcus aureus, spectrum $\beta$-lactamase producing Escherichia coli, and methicillin-resistant Acinetobacter sp., Klebsiella pneumoniae, and Pseudomonas aureginosa (Dharmaratne et al., 2018). The minimum inhibitory concentration values showed that all aqueous and methanol extracts have antibacterial activity with values ranging from 0.25 to $4 \mathrm{mg} / \mathrm{mL}$ against all strains tested, which indicates that further studies are needed to test the antimicrobial potential of Terminalia sp. extracts before designing broad-spectrum antibacterial drugs based of Terminalia sp. (Dharmaratne et al., 2018)

T. ferdinandiana Exell has also been studied for their potential antibacterial properties to evaluate their inhibitory capacity against odor-forming bacteria like Corynebacterium jeikeium, Staphylococcus epidermidis, Propionibacterium acnes, and Brevibacterium linens (Mcmanus et al., 2017). Methanolic extracts from leaves of T. ferdinandiana Exell showed the lowest minimum inhibitory capacity values against C. jeikeium $(233 \mu \mathrm{g} /$ $\mathrm{mL})$, S. epidermidis $(220 \mu \mathrm{g} / \mathrm{mL}), P$. acnes $(625 \mu \mathrm{g} / \mathrm{mL})$, and $\mathrm{B}$ linens $(523 \mu \mathrm{g} / \mathrm{mL})$. Moreover, Mcmanus et al. (2017) determined that leaf extracts of $T$. ferdinandiana Exell were non-toxic by the Artemia franciscana bioassay, interestingly; chloroform and hexane fruit and leaves extracts showed no toxicity activity. LC-MS analysis showed that methanolic extracts of T. ferdinandiana Exell contained some tannins and other compounds, the most abundant were (117) ellagic acid dihydrate, (76) chebulic acid, (4) ellagic acid, (44) castalagin, (5) corilagin, (91) punicalin, (48) luteolin, (9) chebulinic acid, (119) exifone, (53) chebulagic acid, and (120) trimethyl ellagic acid (Mcmanus et al., 2017).

Lee et al. (2017), reported the potential use of ethanol extracts from fruits of Terminalia chebula Retz. to prevent dental plaque bacteria-mediated periodontal disease for their Streptococcus mutans and Aggregatibacter actinomycetemcomitans growthinhibitory capacity. Moreover, concomitant with the anti- 
inflammatory activity of $T$. chebula Retz. extracts, as exhibited by their inhibitory capacity of PGE2 and COX-2. A mixture prepared with $5 \mathrm{mg} / \mathrm{mL}$ A. malaccensis and $20 \mathrm{mg} / \mathrm{mL} T$. catappa L. was evaluated for its antimicrobial properties against $L$. monocytogenes and $S$. aureus, in vacuum packed ready-to-cook chicken, inhibited the growth of $S$. aureus with $1.80,2.13,2.36$, and $2.97 \log \mathrm{CFU} / \mathrm{g}$ reduction in 3,6,9, and 12 days, and also decreased the growth of $L$. monocytogenes with $1.22,1.60$, and $1.55 \log$ CFU reduction at 6,9, and 12 days, respectively. These results may indicate the ability of $A$. malaccensis and T. catappa $L$. extracts to extend shelf-life of chicken meat in vacuum packed ready-to-cook chicken (Somarathna et al., 2018).

Terminalia sp. extracts have been the center of studies regarding the biogenic synthesis of nanoparticles with therapeutic potential. For instance, Sivamaruthi et al. (2019), aimed to biosynthesize silver Palladium bimetallic nanoparticles from aqueous fruit extract of Terminalia chebula Retz., as potential antimicrobial and anticancer agents. The nanoparticles exhibited antimicrobial activity against grampositive bacterial strains like methicillin-resistant $S$. aureus MSRA 11 and MRSA56; and gram-negative bacteria $P$. aeruginosa with a zone of inhibition from $12-16 \mathrm{mM}$ at a concentration of $30 \mu \mathrm{g} / \mathrm{mL}$. Further toxicity studies showed no cytotoxicity in peripheral blood mononuclear cells, even at the highest dose of nanoparticles of $200 \mu \mathrm{g} / \mathrm{mL}$ (Sivamaruthi et al., 2019). Akhter et al. (2019), evaluated the antibacterial activity of biogenic synthesized nanoparticles of zinc, copper, and iron oxides using the extract of Terminalia bellirica (Gaertn.) Roxb. fruits, against gram-positive Staphylococcus aureus, and gramnegative Bacillus subtilis, Escheerichia coli, Klebsiella pneumoniae, and Salmonella enterica. The zone of inhibition of the evaluated nanoparticles exhibited its maximum values for zinc oxide nanoparticles.

Furthermore, studies using T. bellirica (Gaertn.) Roxb. fruit extracts to biosynthesize gold nanoparticles have been reported by Annavaram et al. (2017), who evaluated their antifungal potential. The authors showed that gold nanoparticles against Candida tropicalis and Candida albicans exhibited maximum zone of inhibition of around 16 and $14 \mathrm{~mm}$, respectively. This effect was partially attributed to the presence of (3) gallic acid, (4) ellagic acid, (70) methyl gallate, (37) $\beta$-sitosterol, and (53) chebulagic acid. Figure 4(v), shows the chemical structure of some important compounds with antimicrobial properties.

\section{Antibacterial Activity of Terminalia sp.}

As shown in Table 7, Terminalia superba Engl. \& Diels bark extract inhibited diarrhea-causing pathogens including Shigella dysenteriae and Salmonella typhi (Kuete et al., 2010). The bark, fruit, and seed coat extracts of Terminalia ferdinandiana Exell inhibited Bacillus cereus and methicillin-resistant Staphylococcus aureus with inhibition zone ranging from 7-17.8 mm (Akter et al., 2019). Moreover, the extracts of Terminalia sp. members possessed antibacterial activity against airborne pathogens. MDR Acinetobacter sp. and the growth of MDR Pseudomonas aeruginosa was suppressed when the bacteria were treated with the fruit extract of Terminalia bellirica (Gaertn.) Roxb.
(Dharmaratne et al., 2018) and Terminalia chebula Retz. (Sharma et al., 2012). In addition, Terminalia glaucescens Planch. ex Benth. root (Gbala and Anibijuwon, 2018) and Terminalia superba Engl. \& Diels bark extract (Kuete et al., 2010) inhibited Klebsiella pneumoniae, a pneumonia-causing agent, with MIC at 0.1 and $0.078 \mathrm{mg} / \mathrm{mL}$, respectively. Interestingly, the bark of Terminalia superba Engl. \& Diels inhibited Mycobacterium tuberculosis with a low MIC value of $0.078 \mathrm{mg} / \mathrm{mL}$ (Kuete et al., 2010). This report suggested that extracts from Terminalia sp. plant species could be used as antibacterial agents against pathogenic bacteria.

\section{Antifungal Activity of Terminalia sp.}

Filamentous fungi such as Aspergillus flavus, A. niger, and Trichophyton rubrum are some of the important human pathogens. Besides, opportunistic yeast fungi are an important cause of morbidity and mortality in immunocompromised patients. As shown in Table 8, the growth of filamentous fungi, including $A$. flavus and $A$. niger was inhibited by Terminalia glaucescens Planch. ex Benth. stem extract (Adeeyo et al., 2018). Kuete and his team have shown that the extract from the bark of Terminalia superba Engl. \& Diels possessed antifungal activity against dermatophytes. The MIC values of the extract against Microsporum audouinii and Trichophyton rubrum were 0.019 and $0.039 \mathrm{mg} / \mathrm{mL}$ respectively (Kuete et al., 2010). Terminalia arjuna (Roxb. ex DC.) Wight \& Arn. (Saivaraj and Chandramohan, 2018) and Terminalia catappa L. (Gonçalves et al., 2019) bark, and Terminalia chebula Retz. fruit (Vidya et al., 2019) extract demonstrated antifungal activity against the opportunistic yeasts including Candida albicans, C. glabrata, C. krusei, and C. tropicalis. Kouassi et al. (2019) reported that Terminalia ivorensis A.Chev. and Terminalia mantaly $\mathrm{H}$. Perrier bark extracts inhibited Fusarium ssp., plant pathogenic fungi, with low MIC values ranging from $0.025-0.050 \mathrm{mg} / \mathrm{mL}$ (Kouassi et al., 2019). Moreover, the leaf extract from Terminalia catappa L. exhibited antibiofilm activity against C. albicans and C. glabrata (Gonçalves et al., 2019). The information indicated that the extracts of Terminalia sp. plant species could be used as antifungal agents against pathogenic fungi.

\section{Antiparasitic Activity of Terminalia sp.}

Parasites, including malaria, are the most significant protozoan disease in the world. In 2018, 228 million cases of malaria worldwide were reported by the World Health Organization. In addition, other parasites such as Haemonchus contortus and Trypanosoma brucei are the main cause of morbidity and mortality in humans. The present study is focused on the antiparasitic activity of Terminalia sp. extract against the important parasites (Table 9). Camara and team reported antimalaria activity of Terminalia albida Scott-Elliot bark extract against Plasmodium falciparum with low $\mathrm{IC}_{50}$ as $1.5 \mu \mathrm{g} / \mathrm{mL}$ (Camara et al., 2019). Moreover, an increase in the survival rate of mice infected with the $P$. berghei was detected when they were treated with the bark extract of Terminalia albida Scott-Elliot (Camara et al., 2019) and Terminalia avicennioides Guill. \& Perr. (Owoloye et al., 2019). Interestingly, Mbouna and the team reported that different parts of Terminalia mantaly H. Perrier 
inhibited P. falciparum. It has been commented that $\mathrm{IC}_{50}$ of Terminalia mantaly H. Perrier leaf, root, and stem were 2.09, 7.01, and $3.63 \mu \mathrm{g} / \mathrm{mL}$, respectively (Mbouna et al., 2018). It has been reported that Terminalia superba Engl. \& Diels bark, root, and stem possess antitrypanosomal activity against Trypanosoma brucei brucei with MIC ranging from 0.8-1.6 mg/mL (Antia et al., 2009). Also, the activity of Terminalia arjuna (Roxb. ex DC.) Wight \& Arn. bark extract against $H$. contortus, barber's pole worm, ova, and larva has been documented (Bachaya et al., 2009). This study showed that extracts from Terminalia sp. plant species could be used as alternative agents for the treatment of parasitic infection.

\section{NANOPARTICLES SYNTHESIZED USING TERMINALIA SP. EXTRACTS: IMPROVEMENT OF THE BIO-ACTIVITY}

In an attempt to improve the bioactivity of medicinal plants, much research has focused on nanoparticles to reduce the size of particles and increase the surface area. Plant extracts are capping agents capable of reducing metal ion, resulting in the formation of nanoparticles with remarkable antibacterial activities (Majoumouo et al., 2019). Silver nanoparticles (AgNPs) using Terminalia arjuna (Roxb. ex DC.) Wight \& Arn. bark extract (TA-AgNPs) were synthesized eco-friendly. The antibacterial activity of TA-AgNPs against $E$. coli was better than the extract (Ahmed et al., 2017). MIC values of Terminalia mantaly $\mathrm{H}$. Perrier extract and Terminalia mantaly-AgNPs (TM-AgNPs) against Haemophilus influenzae was reported to be 125 and 3.12 $\mu \mathrm{g} / \mathrm{mL}$, respectively. It was noted that the MIC values of the TMAgNPs were 40 times lower than those of the extract (Majoumouo et al., 2019). Hence, plant-nanoparticle agents are a significant strategy for the treatment of bacterial infection. Terminalia chebula Retz. leaf gold nanoparticles (TC-AgNPs) showed antibacterial activity against $S$. aureus and E. coli. The prepared TC-AgNPs were used on Nylon fabrics for future applying of medical materials.

Interestingly, Nylon cloth-TC-AgNPs exhibited antibacterial activity against organisms with a strength of 3 to 13 times greater than TC-AgNPs (Rohaeti and Rakhmawati, 2017). Gold nanoparticles using Terminalia arjuna (Roxb. ex DC.) Wight \& Arn. leaf extract has been reported to induce mitotic cell division and pollen germination. Moreover, the gold nanoparticles showed a non-cytotoxic effect on root tip cells of Allium cepa and pollen grains of Gloriosa superba (Gopinath et al., 2013). AgNPs synthesized using the polyphenol-rich ethyl acetate fraction of Terminalia bellirica (Gaertn.) Roxb. fruit pericarp, exhibited anticancer activity. Nanoparticles at $120 \mu \mathrm{g} / \mathrm{mL}$ have been reported to kill $69.1 \%$ of colon cancer cells and $65.2 \%$ breast cancer cells. Interestingly, AgNPs did not cause cytotoxic effects against normal cardiac and skeletal muscle cells (Nampoothiri et al., 2018). This information may indicate that the agents of plantnanoparticles are a pronounced strategy to improve the bio-activity of medicinal plants in the treatment of many diseases. Table 10 shows the bioactive potential of several types of nanoparticles synthesized using the extracts of Terminalia sp.

\section{POSSIBLE MECHANISM OF ACTION OF EXTRACTS OF TERMINALIA SP. FOR VARIOUS PHARMACOLOGICAL ACTIVITIES}

Triphala, a common Ayurvedic formulation which consists of the powder of 3 plants of Terminalia sp. such as Phyllanthus emblica L., Terminalia chebula Retz. and Terminalia bellirica (Gaertn.) Roxb., has been used for long times in the traditional system of medicine for the treatment and prevention of ailments that worry the aging population, and its preclinical studies have confirmed most of its ethnomedicinal claims which are mediated by the myriad biochemical mechanisms (Baliga et al., 2015). In 2002, Saleem et al. (2002) have investigated the cytotoxicity potential of fruit extracts of $T$. chebula Retz., which according to him can decrease the number of cells in immortalized and cancer cell lines by preventing the proliferation rate of the cell and by inducing the cell death. His group stated that at lower concentration, the extract was able to induce the cellular pathways that resulted in the apoptosis process, whereas at the higher concentrations, the extract showed direct toxic effects, resulting in the rapid necrotic cell death (Saleem et al., 2002). Leaf extracts of Terminalia muelleri Benth. was reported to show inhibitory potential against the Staphylococcus aureus and the authors also stated that the leaf extract induced the shrinkage and thinning of the cell wall mechanism resulting in the inhibition activity (Anam et al., 2010; Cock, 2015). T. paniculata Roth extracts have been reported to alter the levels of biomarkers of hepatotoxicity in vivo, indicating a hepatoprotective activity. The extracts of $T$. paniculata Roth have been reported to exhibit antioxidant and hepatoprotective activities by altering the levels of biomarkers of hepatotoxicity under in vivo condition, thus protecting the liver by blocking the lipid peroxidation process which could damage the internal tissues (Eesha et al., 2011; Cock, 2015). Alzheimer's disease is considered debilitating dementia, and only a few therapeutic possibilities are presently existing to alter the expressions of the disease and among them, the extracts of $T$. chebula Retz. has been documented to possesses pharmacological activities pertinent to the dementia treatment. The possible anti alzheimer's desease mechanism of action through the anticholinesterase, antiinflammatory, and antioxidant properties of $T$. chebula Retz. has been propose (Afshari et al., 2016) (Figure 5).

In a study, Yeh et al. (2014) have demonstrated that the Terminalia catappa L. leaf extract exhibited an inhibitory effect on several vital steps of metastasis, that includes invasion and migration of cell, by regulating the actions and protein level of urokinase-type plasminogen activator and its natural inhibitor. They also showed that the plant extract could be able to effectively inhibit the phosphorylation of the ERK1/2 signaling pathways by the downregulation of the transcription factors SP-1 and NF- $\mathrm{KB}$ DNA binding activities, that leads to the suppression of urokinase-type plasminogen activator and inhibition of metastasis (Yeh et al., 2014). In another study, Pinheiro Silva et al. (2015), showed that the aqueous fraction from the leaves of Terminalia catappa L. possesses anti Helicobacter pylori activity 
and excellent preventive and curative activity on the acute and chronically induced gastric ulcers. The detailed mechanism of action associated in the gastro-protection are connected to the nitric oxide pathway, an increase in the mucus level and the endogenous prostaglandins, and this fraction was able to cure the ulcers through the inhibition of the matrix metallo proteinase activities (MMP-2 and MMP-9) (Pinheiro Silva et al., 2015).

\section{PRECLINICAL AND CLINICAL STUDIES ON ACTIVE COMPOUNDS FROM TERMINALIA SP.}

\section{Preclinical Studies}

There are currently few preclinical and clinical studies of Terminalia species, as far as our literature research. Out of the few reports, Ekambaram et al. (2018), evaluated the acute toxicity of $T$. chebula Retz. fruit hydrolyzable tannin-rich methanolic extract, which did not exhibit any toxicity even at a dose administration of $5000 \mathrm{mg} / \mathrm{kg} / \mathrm{p}$.o. for 14 days. Thus, Terminalia chebula Retz. fruit extracts can be considered as safe. HPLC analysis showed the presence of (53) chebulagic acid, (5) corilagin, and (9) chebulinic acid. On the other hand, Terminalia sp. extracts significantly affected some biochemical parameters in both male and female rats, which cause a reduction in body weight. This effect could be attributed to the relationship between high tannin content in T. chebula Retz. extracts and their influence on decreased feed intake, growth rate, feed efficiency, net metabolizable energy, and protein digestibility (Ekambaram et al., 2018).

Similarly, Awotunde et al. (2019), evaluated the subacute toxicity of Terminalia schimperiana Hochst. ex Engl. \& Diels (synonym of Terminalia glaucescens Planch. ex Benth.), water extracts in male rats. It was reported that extracts did not have a toxic effect in any organs at doses of 1000, 2000, and $3000 \mathrm{mg} / \mathrm{kg}$ body weight, nor had any effect on the biochemical parameters of treated rats. Also, Das et al. (2015), reported the acute and subacute toxicity of methanol extracts of Terminalia citrina (Gaertn.) Roxb. leaves in female Sprague rats at doses of 250, 500 , and $1000 \mathrm{mg} / \mathrm{kg}$ bodyweight for 28 days. Terminalia sp. treatment had no significant effect on biochemical parameters like alanine aminotransferase, aspartate aminotransferase, alkaline phosphatase, glucose, and creatinine, which indicates no detriment of liver and kidney functions. Also, T. citrina (Gaertn.) Roxb. extracts did not affect hematological parameters such as white blood count, red blood cell count, platelet count and hemoglobin content, neutrophil, lymphocyte, monocyte, eosinophil, and hematocrit content (Das et al., 2015).

\section{Clinical Studies}

Clinical studies regarding the potential bioactivities of Terminalia species are scarce, which limits its potential use as a biopharmaceutical agent against several diseases. Here we briefly summarize the available reports. C. U. Kumar et al. (2015), performed a randomized, double-blind, placebocontrolled, cross over study to evaluate the analgesic activity and safety of a single oral administration of Terminalia chebula Retz. using a hot air pain model in 14 healthy human participants (18-45 years old). The authors administered a single dose of two capsules of $500 \mathrm{mg}$ of an aqueous extract of fruits of Terminalia chebula Retz., which contained no less than $15 \%$ (9) chebulinic acid, $10 \%$ of (53) chebulagic acid and not less than $15 \%$ of other low molecular weight hydrolyzable tannins. $T$. chebula Retz. increased the mean percentage change of pain threshold time and pain tolerance time compared to placebo treatments. This effect was mainly attributed to the antiinflammatory activity of (3) gallic acid, (4) ellagic acid, and (5) corilagin from extracts of fruits of T. chebula Retz. and the antiarthritic effect of the hydrolyzable tannins of Terminalia sp.

Three studies evaluated the effect of Terminalia arjuna (Roxb. ex DC.) Wight \& Arn. in patients with cardiovascular diseases (Kapoor et al., 2015; Maulik et al., 2016; Priya et al., 2019). The report of Priya et al. (2019), found that systolic and diastolic blood pressure decreased after 1 month of T. arjuna (Roxb. ex DC.) Wight \& Arn. therapy, which consisted of the oral administration of $3 \mathrm{~g}$ of a mixture of T. arjuna (Roxb. ex DC.) Wight \& Arn. mixed in $250 \mathrm{~mL}$ of boiled milk twice daily. The bioactive effect was attributed to the presence of adrenergic $\beta 2$ receptor agonistic action. This may be partially related to the antioxidant activity of T. arjuna (Roxb. ex DC.) Wight \& Arn., possibly related to the protection of myocardial ischemic reperfusion injury. Terminalia arjuna (Roxb. ex DC.) Wight \& Arn. treatment also decreased total cholesterol levels (235.02 to $210.80 \mathrm{mg} / \mathrm{dL}$ ) and serum LDL levels (134.40 to 121.5 $\mathrm{mg} / \mathrm{dL}$ ). The second study was conducted as a double-blind, parallel, randomized, placebo-controlled add-on clinical trial by Maulik et al. (2016) to assess the safety of a standardized water extract of stem bark of T. arjuna (Roxb. ex DC.) Wight \& Arn. in chronic heart failure patients.

Nevertheless, the T. arjuna (Roxb. ex DC.) Wight \& Arn. treatment, even when it was well-tolerated, did not change the left ventricular ejection fraction or secondary outcome measures. The third report evaluated the cardioprotective effect of T. arjuna (Roxb. ex DC.) Wight \& Arn. on classical and immuno-inflammatory markers in coronary artery disease by administering $500 \mathrm{mg}$ twice a day to eight patients. Terminalia sp. treatment significantly downregulated the triglycerides, VLDL-C, and immune-inflammatory markers in stable coronary artery disease after 3 months, and the effect was maintained after 6 months with decreased total cholesterol levels.

\section{CONCLUDING REMARKS AND PERSPECTIVES}

Extracts from plants of the genus Terminalia sp. are a rich source of phytochemicals such as terpenes, flavonoids, and phenolic acids. It is suggested that these molecules are related to the antibacterial, antioxidant, antiinflammatory, antifungal, antiviral, antiparasitic, antidiabetic, and anticancer activity of Terminalia plants. Several reports have associated the ethnopharmacological potential of plant extracts or phytochemicals isolated from medicinal plants and plant foods with their bioavailability. However, as far as our literature 
research, we did not find bioavailability reports as well as any pharmacokinetic data. Thus, it is unknown if these molecules will exert any bioactivity in humans.

Essentially, Terminalia plants are yet to be actively explored on the molecular and docking scales, in this case, further exploration of the mechanisms involved in their enzymes modulation and radical scavenging abilities is worth considering. Also, there is little clinical research on the bioactivity of Terminalia species, which practically limits their potential use as a pharmaceutical against diseases. More preclinical and clinical studies are needed if extracts or isolated compounds from Terminalia species want to be used as biopharmaceutical agents. There is still a need for pharmacokinetic and toxicological studies to be able to determine if Terminalia sp. is suitable for the development of a drug or herbal-based remedy; there is also a huge lack of studies regarding the effective doses of Terminalia sp. for prevention/treatment of the pathologies mentioned above. Also, there is an important flaw in the diversity of studies presented here, as the reports lack reproducibility because bioactivity studies use different concentrations of fruit, bark, and leaves Terminalia sp. extracts, nor they use the same plant parts.

\section{AUTHOR CONTRIBUTIONS}

JKP and GD conceptualized the whole concept. JKP, GD, D-YK, CF, EG-G, JH, VN, WM, MP, MN, AS, and RN wrote, reviewed, and edited the manuscript. JKP, HS-S, and BS helped in the collection of literature, review, and editing of the manuscript. All authors contributed to the article and approved the submitted version.

\section{FUNDING}

This work was supported by Korea Institute of Planning and Evaluation for Technology in Food, Agriculture and Forestry

\section{REFERENCES}

Abiodun, O., Gbotosho, G., Ajaiyeoba, E., Happi, T., Falade, M., Wittlin, S., et al. (2011). In vitro antiplasmodial activity and toxicity assessment of some plants from Nigerian ethnomedicine. Pharm. Biol. 49, 9-14. doi: 10.3109/ 13880209.2010.490224

Abiodun, O. O., Gbotosho, G. O., Ajaiyeoba, E. O., Brun, R., and Oduola, A. M. (2012). Antitrypanosomal activity of some medicinal plants from Nigerian ethnomedicine. Parasitol. Res. 110, 521-526. doi: 10.1007/s00436-011-2516-Z

Abraham, A., Mathew, L., and Samuel, S. (2014). Pharmacognostic studies of the fruits of Terminalia bellirica (Gaertn.) Roxb. J. Pharmacognosy Phytochem. 3, 45-52.

Acharyya, S., and Prasenjit Bhuniya, A. S. (2019). Evaluation of antimicrobial and anthelmintic activity of roots of Terminalia paniculata. Pharma. Innovation 8, 1065-1068.

Adeeyo, A. O., Odiyo, J., and Odelade, K. (2018). Chemical profiling and antimicrobial properties of phyto-active extracts from Terminalia glaucescens stem against water microbial contaminants. Open Biotechnol. J. 12, 1-15. doi: 10.2174/1874070701812010001

Afshari, A. R., Sadeghnia, H. R., and Mollazadeh, H. (2016). A review on potential mechanisms of Terminalia chebula in Alzheimer's disease. Adv. Pharmacol. Sci. 2016, 1-15. doi: 10.1155/2016/8964849

Ahmed, Q., Gupta, N., Kumar, A., and Nimesh, S. (2017). Antibacterial efficacy of silver nanoparticles synthesized employing Terminalia arjuna bark extract.
(IPET) through Innovative Food Product and Natural Food Materials Development Program (No.319049-3), funded by Ministry of Agriculture, Food and Rural Affairs (MAFRA).

\section{ACKNOWLEDGMENTS}

The authors are grateful to their respective institutions for support. GD, D-YK, HS-S, and JKP are grateful to Dongguk University, the Republic of Korea for support. D-YK acknowledges the Korea Institute of Planning and Evaluation for Technology in Food, Agriculture and Forestry (IPET) through Innovative Food Product and Natural Food Materials Development Program (No.319049-3), funded by Ministry of Agriculture, Food and Rural Affairs (MAFRA) for support. JKP acknowledges the National Research Foundation of Korea (NRF) grant funded by the Korea government (MSIT) (No. 2020R1G1A1004667), Republic of Korea for support. VN is grateful to the project entitled Medicinal under-exploited Thai native plants against Acanthamoeba, Leishmania donovani, and Plasmodium falciparum-Toward South East Asia collaboration initiative (Grant No. 040226) supported by The Royal Patronage of Her Royal Highness Princess Maha Chakri Sirindhorn for support. MP thanks to project CICECO-Aveiro Institute of Materials, UIDB/50011/2020 \& UIDP/50011/2020, national funds through the Portuguese Foundation for Science and Technology/MCTES.

\section{SUPPLEMENTARY MATERIAL}

The Supplementary Material for this article can be found online at: https://www.frontiersin.org/articles/10.3389/fphar.2020. 561248/full\#supplementary-material

Artif. cells Nanomed. Biotechnol. 45, 1192-1200. doi: 10.1080/21691401. 2016.1215328

Akhter, S. M. H., Mohammad, F., and Ahmad, S. (2019). Terminalia belerica mediated green synthesis of nanoparticles of copper, iron and zinc metal oxides as the alternate antibacterial agents against some common pathogens. BioNanoScience 9, 365-372. doi: 10.1007/s12668-019-0601-4

Akter, S., Netzel, M. E., Tinggi, U., Osborne, S. A., Fletcher, M. T., and Sultanbawa, Y. (2019). antioxidant rich extracts of Terminalia ferdinandiana Inhibit the growth of foodborne bacteria. Foods (Basel Switzerland) 8, 281. doi: 10.3390/foods8080281

Ali, A., Kaur, G., Hamid, H., Abdullah, T., Ali, M., Niwa, M., et al. (2003a). Terminoside A, a new triterpene glycoside from the bark of Terminalia arjuna inhibits nitric oxide production in murine macrophages. J. Asian Natural Prod. Res. 5, 137-142. doi: 10.1080/1028602031000066834

Ali, A., Kaur, G., Hayat, K., Ali, M., and Ather, M. (2003b). A novel naphthanol glycoside from Terminalia arjuna with antioxidant and nitric oxide inhibitory activities. Die Pharmazie 58, 932-934. doi: 10.1002/chin.200413158

Allyn, O. Q., Kusumawati, E., and Nugroho, R. A. (2018). Antimicrobial activity of Terminalia catappa brown leaf extracts against Staphylococcus aureus ATCC 25923 and Pseudomonas aeruginosa ATCC 27853. F1000Research 7, 14061406. doi: 10.12688/f1000research.15998.1

Amalraj, A., and Gopi, S. (2017). Medicinal properties of Terminalia arjuna (Roxb.) Wight \& Arn.: A review. J. Tradit. Complement Med. 7, 65-78. doi: 10.1016/j.jtcme.2016.02.003 
Ambriz-Pérez, D. L., Leyva-López, N., Gutierrez-Grijalva, E. P., and Heredia, J. B. (2016). Phenolic compounds: Natural alternative in inflammation treatment. A Review. Cogent Food Agric. 2, 1-14. doi: 10.1080/23311932.2015.1131412

Anam, K., Suganda, A., Sukandar, E., and Kardono, L. B. S. (2010). Antibacterial effect of component of Terminalia muelleri Benth. against Staphylococcus aureus. IJP-Int. J. Pharmacol. 6, 407-412. doi: 10.3923/ijp.2010.407.412

Aneja, K. R., Sharma, C., and Joshi, R. (2012). Antimicrobial activity of Terminalia arjuna Wight \& Arn.: An ethnomedicinal plant against pathogens causing ear infection. Braz. J. Otorhinolaryngol. 78, 68-74. doi: 10.1590/S180886942012000100011

Anjaneyulu, A., and Prasad, A. R. (1982). Chemical examination of roots or Terminalia-arjuna (ROXB) Wight and Arnot.1. characterization of 2 new triterpenoid glycosides. Indian J. Chem. Section B-Organic Chem. including Med. Chem. 21, 530-533.

Ankamwar, B. (2010). Biosynthesis of gold nanoparticles (green-gold) using leaf extract of Terminalia catappa. J. Chem. 7, 1334-1339. doi: 10.1155/2010/745120

Annavaram, V., Posa, V. R., Vijaya Lakshmi, D., Sumalatha, J., and Somala, A. R. (2017). Terminalia bellirica fruit extract-mediated synthesis of gold nanoparticles (AuNPs) and studies on antimicrobial and antioxidant activity. Inorganic NanoMetal Chem. 47, 681-687. doi: 10.1080/15533174.2016.1212219

Anokwuru, C., Sigidi, M., Boukandou, M., Tshisikhawe, P., Traore, A., and Potgieter, N. (2018). Antioxidant activity and spectroscopic characteristics of extractable and non-extractable phenolics from Terminalia sericea Burch. ex DC. Molecules 23, 1303. doi: 10.3390/molecules23061303

Antia, R., Olayemi, J., Aina, O., and Ajaiyeoba, E. (2009). In vitro and in vivo animal model antitrypanosomal evaluation of ten medicinal plant extracts from south west Nigeria. Afr. J. Biotechnol. 8, 1437-1440.

Anuracpreeda, P., Chankaew, K., Puttarak, P., Koedrith, P., Chawengkirttikul, R., Panyarachun, B., et al. (2016). The anthelmintic effects of the ethanol extract of Terminalia catappa L. leaves against the ruminant gut parasite, Fischoederius cobboldi. Parasitology 143, 421-433. doi: 10.1017/S0031182015001833

Anuradha, V., Shankar, P., Bhuvana, P., Syedali, M., and Yogananth, N. (2017). Terminalia arjuna Bark assisted biosynthesis, characterization and bioactivity of metal oxide nanoparticles. J. Chem. Pharm. Res. 9, 34-46.

Awotunde, O., Adewoye, S., Dhanabal, P., and Hawumba, J. (2019). Subacute toxicity study of aqueous root extract of Terminalia schimperiana in male Wistar rats. Toxicol. Rep. 6, 825-832. doi: 10.1016/j.toxrep.2019.07.006

Bachaya, H. A., Iqbal, Z., Khan, M. N., Jabbar, A., Gilani, A. H., and Din, I.-U. (2009). In vitro and in vivo anthelmintic activity of Terminalia arjuna bark. Int. J. Agric. Biol. 11, 273-278.

Bag, A., Bhattacharyya, S. K., Pal, N. K., and Chattopadhyay, R. R. (2013). Antiinflammatory, antilipid peroxidative, antioxidant and membrane stabilizing activities of hydroalcoholic extract of Terminalia chebula fruits. Pharm. Biol. 51, 1515-1520. doi: 10.3109/13880209.2013.799709

Baliga, M. S., Meera, S., Rai, M. P., Saldanha, E., Pais, S., Jayachander, D., et al. (2015). "Use of the ayurvedic drug triphala in medical conditions afflicting older adults," in Foods and Dietary Supplements in the Prevention and Treatment of Disease in Older Adults (Elsevier), 135-142. doi: 10.1016/C2013-0-00305-2

Basu, T., Panja, S., Ghate, N. B., Chaudhuri, D., and Mandal, N. (2017). Antioxidant and antiproliferative effects of different solvent fractions from Terminalia belerica Roxb. fruit on various cancer cells. Cytotechnology 69, 201216. doi: 10.1007/s10616-016-0051-6

Behl, T., and Kotwani, A. (2017). Proposed mechanisms of Terminalia catappa in hyperglycaemia and associated diabetic complications. J. Pharm. Pharmacol. 69, 123-134. doi: 10.1111/jphp. 12676

Beserra, A.M.S.E.S., Vilegas, W., Tangerina, M. M. P., Ascêncio, S. D., Soares, I. M., Pavan, E., et al. (2018). Chemical characterisation and toxicity assessment In vitro and in vivo of the hydroethanolic extract of Terminalia argentea Mart. leaves. J. Ethnopharmacol. 227, 56-68. doi: 10.1016/j.jep.2018.08.025

Buenz, E. J., Verpoorte, R., and Bauer, B. A. (2018). The ethnopharmacologic contribution to bioprospecting natural products. Annu. Rev. Pharmacol. Toxicol. 58, 509-530. doi: 10.1146/annurev-pharmtox-010617-052703

Busisani, W. L., Kennedy, H. E., Pilani, N., and Eliton, C. (2018). Terminalia Sericea aqueous leaf extract protects growing wistar rats against fructoseinduced fatty liver disease. J. Complement. Integr. Med. 16, 20180035. doi: 10.1515/jcim-2018-0035

Calixto, J. B. (2019). The role of natural products in modern drug discovery. Acad. Bras Cienc. 91 Suppl 3, e20190105. doi: 10.1590/0001-3765201920190105
Camara, A., Haddad, M., Reybier, K., Traoré, M. S., Baldé, M. A., Royo, J., et al. (2019). Terminalia albida treatment improves survival in experimental cerebral malaria through reactive oxygen species scavenging and anti-inflammatory properties. Malaria J. 18, 431-431. doi: 10.1186/s12936-019-3071-9

Camps, J., and García-Heredia, A. (2014). Introduction: oxidation and inflammation, a molecular link between non-communicable diseases," in Oxidative Stress and Inflammation in Non-communicable Diseases-Molecular Mechanisms and Perspectives in Therapeutics. (Cham: Springer). Adv. Exp. Med. Biol. vol 824. doi: 10.1007/978-3-319-07320-0_1

Carluccio, M. A., Siculella, L., Ancora, M. A., Massaro, M., Scoditti, E., Storelli, C., et al. (2003). Olive oil and red wine antioxidant polyphenols inhibit endothelial activation: antiatherogenic properties of Mediterranean diet phytochemicals. Arteriosc. Thromb. Vasc. Biol. 23, 622-629. doi: 10.1161/01.ATV.0000062884.69432.A0

Chandra Sekhar, Y., Phani Kumar, G., and Anilakumar, K. R. (2017). Terminalia arjuna bark extract attenuates picrotoxin-induced behavioral changes by activation of serotonergic, dopaminergic, GABAergic and antioxidant systems. Chin. J. Natural Medicines 15, 584-596. doi: 10.1016/S1875-5364(17)30086-9

Chang, Z., Zhang, Q., Liang, W., Zhou, K., Jian, P., She, G., et al. (2019). A comprehensive review of the structure elucidation of tannins from Terminalia Linn. Evidence-Based Complement. Altern. Med. 2019, 8623909. doi: 10.1155/ 2019/8623909

Cheesman, M. J., White, A., Matthews, B., and Cock, I. E. (2019). Terminalia ferdinandiana fruit and leaf extracts inhibit methicillin-resistant Staphylococcus aureus growth. Planta Med. 85, 1253-1262. doi: 10.1055/a-1013-0434

Cock, I. E., and Rayan, P. (2020). Ascorbic acid potentiates the Giardia duodenalis growth inhibitory activity of pure Terminalia ferdinandiana Exell compounds. Parasitol. Res. 119, 1125-1137. doi: 10.1007/s00436-019-06579-1

Cock, I. (2015). The medicinal properties and phytochemistry of plants of the genus Terminalia (Combretaceae). Inflammopharmacology 23, 203-229. doi: 10.1007/s10787-015-0246-z

Cota, D., Mishra, S., and Shengule, S. (2019). Beneficial role of Terminalia arjuna hydro-alcoholic extract in colitis and its possible mechanism. J. Ethnopharmacol. 230, 117-125. doi: 10.1016/j.jep.2018.10.020

Cunningham, A., Garnett, S., Gorman, J., Courtenay, K., and Boehme, D. (2009). Eco-enterprises and terminalia ferdinandiana: "best laid plans" and Australian policy lessons. Econ. Bot. 63, 16-28. doi: 10.1007/s12231-008-9055-2

Das, N., Goshwami, D., Hasan, M. S., and Raihan, S. Z. (2015). Evaluation of acute and subacute toxicity induced by methanol extract of Terminalia citrina leaves in Sprague Dawley rats. J. Acute Dis. 4, 316-321. doi: 10.1016/j.joad.2015.05.001

Dawe, A., Talom, B., Kapche, G., Siddiqui, K., Yakai, F., Talla, E., et al. (2017). Termiglaucescin, a new polyhydroxy triterpene glucoside from Terminalia glaucescens with antioxidant and antiinflammatory potential. Z. Fur Naturforschung Section C-a J. Biosci. 72, 203-208.

Debnath, S., Dey, D., Hazra, S., Ghosh, S., Ray, R., and Hazra, B. (2013). Antibacterial and antifungal activity of Terminalia arjuna Wight \& Arn. bark against multi-drug resistant clinical isolates. J. Coastal Life Med. 1, 315-321. doi: 10.1515/znc-20160178

Defronzo, R. A., Ferrannini, E., Zimmet, P., and Alberti, G. (2015). International Textbook of Diabetes Mellitus, 2 Volume Set (John Wiley \& Sons).

Dharmaratne, M. P. J., Manoraj, A., Thevanesam, V., Ekanayake, A., Kumar, N. S., Liyanapathirana, V., et al. (2018). Terminalia bellirica fruit extracts: in-vitro antibacterial activity against selected multidrug-resistant bacteria, radical scavenging activity and cytotoxicity study on BHK-21 cells. BMC Complement. Altern. Med. 18, 325-325. doi: 10.1186/s12906-018-2382-7

Dwevedi, A., Dwivedi, R., and Sharma, Y. K. (2016). Exploration of phytochemicals found in Terminalia sp. and their antiretroviral activities. Pharmacognosy Rev. 10, 73-83. doi: 10.4103/0973-7847.194048

Dwivedi, S., and Chopra, D. (2014). Revisiting Terminalia arjuna - An Ancient Cardiovascular Drug. J. Tradit. Complement. Med. 4, 224-231. doi: 10.4103/ 2225-4110.139103

Eesha, B., Mohanbabu, A. V., Meena, K. K., Vijay, M., Lalit, M., and Rajput, R. (2011). Hepatoprotective activity of Terminalia paniculata against paracetamol induced hepatocellular damage in Wistar albino rats. Asian Pacific J. Trop. Med. 4, 466-469. doi: 10.1016/S1995-7645(11)60127-2

Ekambaram, S. P., Babu, K. B., Perumal, S. S., and Rajendran, D. (2018). Repeated oral dose toxicity study on hydrolysable tannin rich fraction isolated from fruit pericarps of Terminalia chebula Retz in Wistar albino rats. Regul. Toxicol. Pharmacol. 92, 182-188. doi: 10.1016/j.yrtph.2017.12.001 
Eloff, J. N., Katerere, D. R., and Mcgaw, L. J. (2008). The biological activity and chemistry of the southern African Combretaceae. J. Ethnopharmacol. 119, 686699. doi: 10.1016/j.jep.2008.07.051

Elvire, K. F. E., Casimir, A. D., Durand, D.-N., Bawa, B., Lamine, B. M., and Frédéric, L. (2018). Antioxidant and Antibacterial Activities of Terminalia superba Engl. and Diels (Combretaceae) bark extracts. Int. J. Curr. Microbiol. Appl. Sci. 7, 2836-2846. doi: 10.20546/ijcmas.2018.707.332

Fahmy, N. M., Al-Sayed, E., Abdel-Daim, M. M., and Singab, A. N. (2017). Antiinflammatory and analgesic activities of Terminalia muelleri Benth. (Combretaceae). Drug Dev. Res. 78, 146-154. doi: 10.1002/ddr.21385

Fan, C., Dong, Y., Xie, Y., Su, Y., Zhang, X., Leavesley, D., et al. (2015). Shikonin reduces TGF- $\beta 1$-induced collagen production and contraction in hypertrophic scar-derived human skin fibroblasts. Int. J. Mol. Med. 36, 985-991. doi: 10.3892/ijmm.2015.2299

Fuhrman, B., and Aviram, M. (2001). Antiatherogenecity of nutritional compounds. Invest. Drug J. 4, 82-92.

Garai, C., Hasan, S. N., Barai, A. C., Ghorai, S., Panja, S. K., and Bag, B. G. (2018). Green synthesis of Terminalia arjuna-conjugated palladium nanoparticles (TA-PdNPs) and its catalytic applications. J. Nanostruct. Chem. 8, 465-472. doi: 10.1007/s40097-018-0288-z

Gautam, R., and Jachak, S. M. (2009). Recent developments in antiinflammatory natural products. Med. Res. Rev. 29, 767-820. doi: 10.1002/med.20156

Gautam, M. K., Goel, S., Ghatule, R. R., Singh, A., Nath, G., and Goel, R. K. (2013). Curative effect of Terminalia chebula extract on acetic acid-induced experimental colitis: role of antioxidants, free radicals and acute inflammatory marker. Inflammopharmacology 21, 377-383. doi: 10.1007/s10787-012-0147-3

Gbala, I., and Anibijuwon, I. (2018). Antibacterial activity of Terminalia glaucescens, Mangifera indica and Mitracarpus villosus on carbapenemresistant enterobacteriaceae. Afr. J. Clin. Exp. Microbiol. 19, 251-259. doi: 10.4314/ajcem.v19i4.2

Ghate, N. B., Hazra, B., Sarkar, R., Chaudhuri, D., and Mandal, N. (2014). Alteration of $\mathrm{Bax} / \mathrm{Bcl}-2$ ratio contributes to Terminalia belerica-induced apoptosis in human lung and breast carcinoma. In Vitro Cell. Dev. Biol. Anim. 50, 527-537. doi: 10.1007/s11626-013-9726-x

Ghosh, S., Banerjee, S., and Sil, P. C. (2015). The beneficial role of curcumin on inflammation, diabetes and neurodegenerative disease: A recent update. Food Chem. Toxicol. 83, 111-124. doi: 10.1016/j.fct.2015.05.022

Gonçalves, L. M., Madeira, P. L. B., Diniz, R. S., Nonato, R. F., Siqueira, F. S. F. D., De Sousa, E. M., et al. (2019). Effect of Terminalia catappa Linn. on Biofilms of Candida albicans and Candida glabrata and on changes in color and roughness of acrylic resin. Evidence-Based Complement. Altern. Med. 2019, 1-8. doi: 10.1155/ 2019/7481341

Gopinath, K., Venkatesh, K. S., Ilangovan, R., Sankaranarayanan, K., and Arumugam, A. (2013). Green synthesis of gold nanoparticles from leaf extract of Terminalia arjuna, for the enhanced mitotic cell division and pollen germination activity. Ind. Crops Prod. 50, 737-742. doi: 10.1016/ j.indcrop.2013.08.060

Haidara, M., Haddad, M., Denou, A., Marti, G., Bourgeade-Delmas, S., Sanogo, R., et al. (2018). In vivo validation of antimalarial activity of crude extracts of Terminalia macroptera, a Malian medicinal plant. Malaria J. 17, 68-68. doi: 10.1186/s12936-018-2223-7

Halliwell, B., and Gutteridge, J. M. (2015). Free Radicals in Biology and Medicine (USA: Oxford University Press).

Harvey, A. L., Edrada-Ebel, R., and Quinn, R. J. (2015). The re-emergence of natural products for drug discovery in the genomics era. Nat. Rev. Drug Discovery 14, 111-129. doi: 10.1038/nrd4510

Heinrich, M. (2000). Ethnobotany and its role in drug development. Phytother. Res. 14, 479-488. doi: 10.1002/1099-1573(200011)14:7<479::AID-PTR958>3.0.CO;2-2

Huang, Y.-H., Wu, P.-Y., Wen, K.-C., Lin, C.-Y., and Chiang, H.-M. (2018). Protective effects and mechanisms of Terminalia catappa L. methenolic extract on hydrogen-peroxide-induced oxidative stress in human skin fibroblasts. BMC Complement. Altern. Med. 18, 266-266. doi: 10.1186/s12906-018-2308-4

Ingole, A., Kamble, M., Dhabarde, D., Baheti, J., and Bhoge, P. (2019). Assessment of anthelmintic potential of hydroalcoholic fruit flesh extract of Terminalia catappa Linn. J. Drug Delivery Ther. 9, 160-162. doi: 10.22270/jddt.v9i3.2631

International Diabetes Federation (2019). IDF Diabetes Atlas (978-2-930229-87-4) (International Diabetes Federation). Available at: http://www.diabetesatlas.org/.

Intharuksa, A., Ando, H., Miyake, K., Sirisa-Ard, P., Mikage, M., and Sasaki, Y. (2016). Molecular analysis of Terminalia spp. distributed in Thailand and authentication of crude drugs from Terminalia plants. Biol. Pharm. Bull. 39, 492-501. doi: 10.1248/bpb.b15-00673

Jokar, A., Masoomi, F., Sadeghpour, O., Nassiri-Toosi, M., and Hamedi, S. (2016). Potential therapeutic applications for Terminalia chebula in Iranian traditional medicine. J. Tradit. Chin. Med. 36, 250-254. doi: 10.1016/S0254-6272(16)30035-8

Kapoor, D., Vijayvergiya, R., and Dhawan, V. (2014). Terminalia arjuna in coronary artery disease: Ethnopharmacology, pre-clinical, clinical \& safety evaluation. J. Ethnopharmacol. 155, 1029-1045. doi: 10.1016/j.jep.2014.06.056

Kapoor, D., Trikha, D., Vijayvergiya, R., Parashar, K. K., Kaul, D., and Dhawan, V. (2015). Short-term adjuvant therapy with Terminalia arjuna attenuates ongoing inflammation and immune imbalance in patients with stable coronary artery disease: In vitro and in vivo evidence. J. Cardiovasc. Trans. Res. 8, 173-186. doi: 10.1007/s12265-015-9620-x

Khan, M. S. A., Khatoon, N., Al-Sanea, M. M., Mahmoud, M. G., and Rahman, H. U. (2018). Methanolic extract of leathery murdah, Terminalia coriacea (Roxb.) Wight and Arn. leaves exhibits antiinflammatory activity in acute and chronic models. Med. Principles Pract. 27, 267-271. doi: 10.1159/000488199

Khan, M. E., Bala, L. M., and Maliki, M. (2019). Phytochemical Analyses of Terminalia schimperiana (combretaceae) root bark extract to isolate stigmasterol. Adv. J. Chemistry-Section A (Theor. Eng. Appl. Chem.) 2, 327334. doi: 10.33945/SAMI/AJCA.2019.4.6

Kibar Ozturk, M., Zindanc1, I., and Zemheri, E. (2018). Acacia seyal and Terminalia brownii associated airborne contact dermatitis (Dukhan dermatitis). Int. J. Dermatol. 57, 1382-1386. doi: 10.1111/ijd.14194

Kim, H., and Song, M. J. (2013). Ethnomedicinal practices for treating liver disorders of local communities in the southern regions of Korea. Evid. Based Complement Alternat. Med. 2013, 869176. doi: 10.1155/2013/869176

Kim, M.-S., Lee, D. Y., Lee, J., Kim, H. W., Sung, S. H., Han, J.-S., et al. (2018). Terminalia chebula extract prevents scopolamine-induced amnesia via cholinergic modulation and antioxidative effects in mice. BMC Complement. Altern. Med. 18, 136-136. doi: 10.1186/s12906-018-2212-y

Konczak, I., Zabaras, D., Dunstan, M., and Aguas, P. (2010). Antioxidant capacity and hydrophilic phytochemicals in commercially grown native Australian fruits. Food Chem. 123, 1048-1054. doi: 10.1016/j.foodchem.2010.05.060

Konczak, I., Maillot, F., and Dalar, A. (2014). Phytochemical divergence in 45 accessions of Terminalia ferdinandiana (Kakadu plum). Food Chem. 151, 248256. doi: 10.1016/j.foodchem.2013.11.049

Koparde, S., and Gaikwad, D. (2017). Antibacterial potential of green silver nanoparticles synthesized from medicinal plant Terminalia arjuna. Int. J. Res. Biosci. Agric. Technol. 5, 258-260.

Kouassi, E. K., Coulibaly, I., Pop, R., Adela, P., and Allico, J. D. (2019). In vitro antioxidant potency and antifungal efficiency of four local Terminalia species against Fusarium strains. J. Exp. Agric. Int. 29 (2), 1-7. doi: 10.9734/JEAI/2019/ 45047

Kuete, V., Tabopda, T. K., Ngameni, B., Nana, F., Tshikalange, T. E., and Ngadjui, B. T. (2010). Antimycobacterial, antibacterial and antifungal activities of Terminalia superba (Combretaceae). South Afr. J. Bot. 76, 125-131. doi: 10.1016/j.sajb.2009.09.009

Kumar, N., Gangappa, D., Gupta, G., and Karnati, R. (2014). Chebulagic acid from Terminalia chebula causes G1 arrest, inhibits NFKB and induces apoptosis in retinoblastoma cells. BMC Complement. Altern. Med. 14, 319-319. doi: 10.1186/1472-6882-14-319

Kumar, C. U., Pokuri, V. K., and Pingali, U. (2015). Evaluation of the Analgesic activity of standardized aqueous extract of Terminalia chebula in healthy human participants using hot air pain model. J. Clin. Diagn. Res. 9, FC01FC04. doi: 10.7860/JCDR/2015/11369.5916

Kumar, R., Arora, R., Agarwal, A., and Gupta, Y. K. (2018). Protective effect of Terminalia chebula against seizures, seizure-induced cognitive impairment and oxidative stress in experimental models of seizures in rats. J. Ethnopharmacol. 215, 124-131. doi: 10.1016/j.jep.2017.12.008

Kuo, P.-L., Hsu, Y.-L., Lin, T.-C., Lin, L.-T., Chang, J.-K., and Lin, C.-C. (2005). Casuarinin from the bark of Terminalia arjuna induces apoptosis and cell cycle arrest in human breast adenocarcinoma MCF-7 cells. Planta Med. 71, 237-243. doi: 10.1055/s-2005-837823

Kuriakose, J., Lal Raisa, H., Vysakh, A., Eldhose, B., and Latha, M. S. (2017). Terminalia bellirica (Gaertn.) Roxb. fruit mitigates CCl4 induced oxidative stress and hepatotoxicity in rats. Biomed. Pharmacother. 93, 327-333. doi: 10.1016/j.biopha.2017.06.080 
Lago, J. H. G., Toledo-Arruda, A. C., Mernak, M., Barrosa, K. H., Martins, M. A., Tibério, I. F. L. C., et al. (2014). Structure-activity association of flavonoids in lung diseases. Molecules (Basel Switzerland) 19, 3570-3595. doi: 10.3390/ molecules 19033570

Latha, R. C. R., and Daisy, P. (2013). Therapeutic potential of octyl gallate isolated from fruits of Terminalia bellerica in streptozotocin-induced diabetic rats. Pharm. Biol. 51, 798-805. doi: 10.3109/13880209.2013.766894

Lee, Y., Byun, H. S., Seok, J. H., Park, K. A., Won, M., Seo, W., et al. (2016). Terminalia chebula provides protection against dual modes of necroptotic and apoptotic cell death upon death receptor ligation. Sci. Rep. 6, 15. doi: 10.1038/srep25094

Lee, J., Nho, Y. H., Yun, S. K., and Hwang, Y. S. (2017). Use of ethanol extracts of Terminalia chebula to prevent periodontal disease induced by dental plaque bacteria. BMC Complement. Altern. Med. 17, 113-113. doi: 10.1186/s12906017-1619-1

Lee, C. Y., Yang, S. F., Wang, P. H., Su, C. W., Hsu, H. F., Tsai, H. T., et al. (2018). Antimetastatic effects of Terminalia catappa leaf extracts on cervical cancer through the inhibition of matrix metalloprotein-9 and MAPK pathway. Environ. Toxicol. 34, 60-66. doi: 10.1002/tox.22657

Lee, C.-Y., Yang, S.-F., Wang, P.-H., Su, C.-W., Hsu, H.-F., Tsai, H.-T., et al. (2019). Antimetastatic effects of Terminalia catappa leaf extracts on cervical cancer through the inhibition of matrix metalloprotein-9 and MAPK pathway. Environ. Toxicol. 34, 60-66. doi: 10.1002/tox.22657

Li, K., Diao, Y., Zhang, H., Wang, S., Zhang, Z., Yu, B., et al. (2011). Tannin extracts from immature fruits of Terminalia chebula Fructus Retz. promote cutaneous wound healing in rats. BMC Complement. Altern. Med. 11, 86-86. doi: $10.1186 / 1472-6882-11-86$

Lin, T.-C., Chien, S.-C., Chen, H.-F., and Hsu, F.-L. (2000). Tannins and related compounds from Combretaceae plants. Chin. Pharm. J. 52, 1-26.

Lopez, H. L., Habowski, S. M., Sandrock, J. E., Raub, B., Kedia, A., Bruno, E. J., et al. (2017). Effects of dietary supplementation with a standardized aqueous extract of Terminalia chebula fruit (AyuFlex $\left({ }^{\circledR}\right)$ ) on joint mobility, comfort, and functional capacity in healthy overweight subjects: a randomized placebocontrolled clinical trial. BMC Complement. Altern. Med. 17, 475-475. doi: 10.1186/s12906-017-1977-8

Losso, J. N., Bansode, R. R., Trappey Ii, A., Bawadi, H. A., and Truax, R. (2004). In vitro antiproliferative activities of ellagic acid. J. Nutr. Biochem. 15, 672-678. doi: 10.1016/j.jnutbio.2004.06.004

Majoumouo, M. S., Sibuyi, N. R. S., Tincho, M. B., Mbekou, M., Boyom, F. F., and Meyer, M. (2019). Enhanced antibacterial activity of biogenic silver nanoparticles synthesized from Terminalia mantaly Extracts. Int. J. Nanomed. 14, 9031-9046. doi: 10.2147/IJN.S223447

Makihara, H., Shimada, T., Machida, E., Oota, M., Nagamine, R., Tsubata, M., et al. (2012). Preventive effect of Terminalia bellirica on obesity and metabolic disorders in spontaneously obese type 2 diabetic model mice. J. Natural Medicines 66, 459-467. doi: 10.1007/s11418-011-0606-y

Makihara, H., Koike, Y., Ohta, M., Horiguchi-Babamoto, E., Tsubata, M., Kinoshita, K., et al. (2016). Gallic acid, the active ingredient of Terminalia bellirica, enhances adipocyte differentiation and adiponectin secretion. Biol. Pharm. Bull. 39, 1137-1143. doi: 10.1248/bpb.b16-00064

Malterud, K. E. J. P. (2017). Ethnopharmacology, chemistry and biological properties of four Malian medicinal plants. Plants (Basel) 6:11. doi: 10.3390/plants6010011

Marjenah, M., and Putri, N. P. (2017). Morphological characteristic and physical environment of Terminalia catappa in East Kalimantan, Indonesia. Asian J. Forest. 1, 33-39. doi: 10.13057/asianjfor/r010105

Mata, R., Cristians, S., Escandón-Rivera, S., Juárez-Reyes, K., and Rivero-Cruz, I. (2013). Mexican antidiabetic herbs: valuable sources of inhibitors of $\alpha$ glucosidases. J. Natural Prod. 76, 468-483. doi: 10.1021/np300869g

Maulik, S. K., and Katiyar, C. K. (2010). Terminalia arjuna in cardiovascular diseases: making the transition from traditional to modern medicine in India. Curr. Pharm. Biotechnol. 11, 855-860. doi: 10.2174/138920110793262051

Maulik, S. K., and Talwar, K. K. (2012). Therapeutic potential of Terminalia arjuna in cardiovascular disorders. Am. J. Cardiovasc. Drugs 12, 157-163. doi: 10.2165/11598990-000000000-00000

Maulik, S. K., Wilson, V., Seth, S., Bhargava, B., Dua, P., Ramakrishnan, S., et al. (2016). Clinical efficacy of water extract of stem bark of Terminalia arjuna (Roxb. ex DC.) Wight \& Arn. in patients of chronic heart failure: a doubleblind, randomized controlled trial. Phytomedicine 23, 1211-1219. doi: 10.1016/ j.phymed.2016.02.007
Mbosso Teinkela, J. E., Siwe Noundou, X., Fannang, S., Mbem Song, A., Assob Nguedia, J. C., Hoppe, H. C., et al. (2019). Terminaliamide, a new ceramide and other phytoconstituents from the roots of Terminalia mantaly $\mathrm{H}$. Perrier and their biological activities. Natural Prod. Res. 1-10. doi: 10.1080/14786419.2019. 1647425

Mbouna, C. D., Kouipou, R. M., Keumoe, R., Tchokouaha, L. R., Fokou, P. V., Tali, B. M., et al. (2018). Potent antiplasmodial extracts and fractions from Terminalia mantaly and Terminalia superba. Malaria J. 17, 142. doi: 10.1186/s12936-018-2298-1

Mcmanus, K., Wood, A., Wright, M. H., Matthews, B., Greene, A. C., and Cock, I. E. (2017). Terminalia ferdinandiana Exell. Extracts inhibit the growth of body odourforming bacteria. Int. J. Cosmetic Sci. 39, 500-510. doi: 10.1111/ics.12403

Minsakorn, S., Nuplod, K., Puttarak, P., Chawengkirttikul, R., Panyarachun, B., Ngamniyom, A., et al. (2019). The anthelmintic effects of medicinal plant extracts against paramphistome parasites, Carmyerius spatiosus. Acta Parasitol. 64, 566-574. doi: 10.2478/s11686-019-00072-6

Mitra, M., Bandyopadhyay, A., Datta, G., and Nandi, D. (2019). Protective role of green synthesized gold nanoparticles using Terminalia arjuna against acetaminophen induced hematological alterations in male wistar rats. J. Nanomed. Nanotechnol. 10, 1-7. doi: 10.35248/2157-7439.19.10.530

Mohan Kumar, K., Mandal, B. K., Sinha, M., and Krishnakumar, V. (2012). Terminalia chebula mediated green and rapid synthesis of gold nanoparticles. Spectrochim. Acta Part A: Mol. Biomol. Spectrosc. 86, 490-494. doi: 10.1016/j.saa.2011.11.001

Mongalo, N., Mcgaw, L., Segapelo, T., Finnie, J., and Van Staden, J. (2016). Ethnobotany, phytochemistry, toxicology and pharmacological properties of Terminalia sericea Burch. ex DC. (Combretaceae)-A review. J. Ethnopharmacol. 194, 789-802. doi: 10.1016/j.jep.2016.10.072

Mopuri, R., Ganjayi, M., Banavathy, K. S., Parim, B. N., and Meriga, B. (2015). Evaluation of antiobesity activities of ethanolic extract of Terminalia paniculata bark on high fat diet-induced obese rats. BMC Complement. Altern. Med. 15, 76-76. doi: 10.1186/s12906-015-0598-3

Muhit, M. A., Umehara, K., Mori-Yasumoto, K., and Noguchi, H. (2016). Furofuran lignan glucosides with estrogen-inhibitory properties from the bangladeshi medicinal plant Terminalia citrina. J. Natural Prod. 79, 12981307. doi: 10.1021/acs.jnatprod.5b01042

Nair, S., and Nagar, R. (1997). Antioxidant flavonoids in common Indian foods. South Asian J. Prevent. Cardiol. 1, 33-35.

Nair, S., Nagar, R., and Gupta, R. (1996). Dietary antioxidant phenolics and flavonoids in coronary heart disease. Indian Heart J. 48, 545.

Nair, J. J., Aremu, A. O., and Van Staden, J. (2012). Antiinflammatory effects of Terminalia phanerophlebia (Combretaceae) and identification of the active constituent principles. South Afr. J. Bot. 81, 79-80. doi: 10.1016/j.sajb.2012.06.001

Nampoothiri, S. V., Suresh Kumar, B., Esakkidurai, T., and Pitchumani, K. (2018). Green synthesis of silver nanoparticles using a characterized polyphenol rich fraction from Terminalia bellirica and the evaluation of its cytotoxicity in normal and cancer cells. J. Biologically Active Prod. Nat. 8, 352-363. doi: 10.1080/22311866.2018.1540944

National Center for Biotechnology Information (2019a). Arjunolic acid, $C I D=73641$ [Online]. Available at: https://pubchem.ncbi.nlm.nih.gov/ compound/Arjunolic-acid (Accessed January 2nd 2020).

National Center for Biotechnology Information (2019aa). Stigmast-4-ene-3,6dione, $C I D=5490007$ [Online]. Available at: https://pubchem.ncbi.nlm.nih. gov/compound/Stigmast-4-ene-3_6-dione (Accessed January 2nd 2020).

National Center for Biotechnology Information (2019ab). Tannic acid, $C I D=16129778$ [Online]. Available at: https://pubchem.ncbi.nlm.nih.gov/ compound/Tannic-acid (Accessed January 2nd 2020).

National Center for Biotechnology Information (2019ac). Vescalagin, CID=168165 [Online]. Available at: https://pubchem.ncbi.nlm.nih.gov/compound/ Vescalagin (Accessed January 2nd 2020).

National Center for Biotechnology Information (2019ad). Vitexin, CID=5280441 [Online]. Available at: https://pubchem.ncbi.nlm.nih.gov/compound/Vitexin (Accessed January 2nd 2020).

National Center for Biotechnology Information (2019b). beta-Sitosterol, $C I D=222284$ [Online]. Available at: https://pubchem.ncbi.nlm.nih.gov/ compound/beta-Sitosterol (Accessed January 2nd 2020).

National Center for Biotechnology Information (2019c). Chebulagic acid, CID= 442674 [Online]. Available at: https://pubchem.ncbi.nlm.nih.gov/compound/ 442674 (Accessed January 2nd 2020). 
National Center for Biotechnology Information (2019d). Chebulic acid, $C I D=71308174$ [Online]. Available at: https://pubchem.ncbi.nlm.nih.gov/ compound/71308174 (Accessed January 2nd 2020).

National Center for Biotechnology Information (2019e). Cianidanol, CID=9064 [Online]. Available at: https://pubchem.ncbi.nlm.nih.gov/compound/ Cianidanol (Accessed January 2nd 2020).

National Center for Biotechnology Information (2019f). CID $=72284$ [Online]. Available at: https://pubchem.ncbi.nlm.nih.gov/compound/Chebulinic-acid (Accessed January 2nd 2020).

National Center for Biotechnology Information (2019g). CID= 73178 [Online]. Available at: https://pubchem.ncbi.nlm.nih.gov/compound/1_2_3_6-TetrakisO-galloyl-beta-D-glucose (Accessed January 2nd 2020).

National Center for Biotechnology Information (2019h). CID $=44584733$ [Online]. Available at: https://pubchem.ncbi.nlm.nih.gov/compound/44584733 (Accessed January 2nd 2020).

National Center for Biotechnology Information (2019i). Corilagin, $C I D=73568$ [Online]. Available at: https://pubchem.ncbi.nlm.nih.gov/compound/Corilagin (Accessed January 2nd 2020).

National Center for Biotechnology Information (2019j). Ellagic acid dihydrate, $C I D=16760409$ [Online]. Available at: https://pubchem.ncbi.nlm.nih.gov/ compound/Ellagic-acid-dihydrate (Accessed January 2nd 2020).

National Center for Biotechnology Information (2019k). Ellagic acid, $C I D=5281855$ [Online]. Available at: https://pubchem.ncbi.nlm.nih.gov/ compound/Ellagic-acid (Accessed [Accessed January 2nd 2020).

National Center for Biotechnology Information (20191). Exifone, CID=40399 [Online]. Available at: https://pubchem.ncbi.nlm.nih.gov/compound/Exifone (Accessed January 2nd 2020).

National Center for Biotechnology Information (2019m). Gallic acid, CID=370 [Online]. Available at: https://pubchem.ncbi.nlm.nih.gov/compound/Gallicacid (Accessed January 2nd 2020).

National Center for Biotechnology Information (2019n). Geraniin, CID=3001497 [Online]. Available at: https://pubchem.ncbi.nlm.nih.gov/compound/3001497 (Accessed January 2nd 2020).

National Center for Biotechnology Information (2019o). Isoorientin, $C I D=114776$ [Online]. Available at: https://pubchem.ncbi.nlm.nih.gov/compound/ Isoorientin (Accessed January 2nd 2020).

National Center for Biotechnology Information (2019p). Luteolin, CID=5280445 [Online]. Available at: https://pubchem.ncbi.nlm.nih.gov/compound/Luteolin (Accessed January 2nd 2020).

National Center for Biotechnology Information (2019q). Methyl gallate, $C I D=7428$ [Online]. Available at: https://pubchem.ncbi.nlm.nih.gov/ compound/Methyl-gallate (Accessed January 2nd 2020).

National Center for Biotechnology Information (2019r). Octyl gallate, $C I D=61253$ [Online]. Available at: https://pubchem.ncbi.nlm.nih.gov/compound/Octylgallate (Accessed January 2nd 2020).

National Center for Biotechnology Information (2019s). Pentagalloylglucose, $C I D=65238$, [Online]. Available at: https://pubchem.ncbi.nlm.nih.gov/ compound/Pentagalloylglucose (Accessed January 2nd 2020).

National Center for Biotechnology Information (2019t). Punicalin, $C I D=5388496$ [Online]. Available at: https://pubchem.ncbi.nlm.nih.gov/compound/Punicalin (Accessed January 2nd 2020).

National Center for Biotechnology Information (2019u). Purpurin, CID=6683 [Online]. Available at: https://pubchem.ncbi.nlm.nih.gov/compound/Purpurin (Accessed January 2nd 2020).

National Center for Biotechnology Information (2019v). Quercetin, CID=5280343 [Online]. Available at: https://pubchem.ncbi.nlm.nih.gov/compound/ Quercetin (Accessed January 2nd 2020).

National Center for Biotechnology Information (2019w). Reserpine, CID $=5770$ [Online]. Available at: https://pubchem.ncbi.nlm.nih.gov/compound/ Reserpine (Accessed January 2nd 2020).

National Center for Biotechnology Information (2019x). Resveratrol, CID=445154 [Online]. Available at: https://pubchem.ncbi.nlm.nih.gov/compound/ Resveratrol (Accessed January 2nd 2020).

National Center for Biotechnology Information (2019y). Rutin, CID=5280805 [Online]. Available at: https://pubchem.ncbi.nlm.nih.gov/compound/Rutin (Accessed January 2nd 2020).

National Center for Biotechnology Information (2019z). Stigmast-4-en-3-one, $C I D=5484202$ [Online]. Available at: https://pubchem.ncbi.nlm.nih.gov/ compound/Stigmast-4-en-3-one (Accessed January 2nd 2020).
Netzel, M., Netzel, G., Tian, Q., Schwartz, S., and Konczak, I. (2007). Native Australian fruits-a novel source of antioxidants for food. Innovative Food Sci. Emerg. Technol. 8, 339-346. doi: 10.1016/j.ifset.2007.03.007

Nguyen, Q.-V., Nguyen, V. B., Eun, J.-B., Wang, S.-L., Nguyen, D. H., Tran, T. N., et al. (2016). Antioxidant and antidiabetic effect of some medicinal plants belong to Terminalia species collected in Dak Lak Province, Vietnam. Res. Chem. Intermediates 42, 5859-5871. doi: 10.1007/s11164-015-2409-3

Ohno, Y., Fukuda, K., Takemura, G., Toyota, M., Watanabe, M., Yasuda, N., et al. (1999). Induction of apoptosis by gallic acid in lung cancer cells. Anticancer Drugs 10, 845-851. doi: 10.1097/00001813-199910000-00008

Owoloye, A., Akanbi, O. M., and Bakare, O. S. (2019). Effects of Powdered stem bark of Terminalia avicennioides made as dietary feed fed to mice infected with Plasmodium berghei, on liver function. Asian J. Biochem. Genet. Mol. Biol. 2, 111. doi: 10.9734/ajbgmb/2019/v2i430066

Pandey, G., Gupta, S. S., Bhatia, A., Sidhu, O. P., Rawat, A. K. S., and Rao, C. V. (2017). Grilling enhances antidiarrheal activity of Terminalia bellerica Roxb. fruits. J. Ethnopharmacol. 202, 63-66. doi: 10.1016/j.jep.2016.12.003

Pettit, G. R., Hoard, M. S., Doubek, D. L., Schmidt, J. M., Pettit, R. K., Tackett, L. P., et al. (1996). Antineoplastic agents 338. The cancer cell growth inhibitory. constituents of Terminalia arjuna (Combretaceae). J. Ethnopharmacol. 53, 5763. doi: 10.1016/S0378-8741(96)01421-3

Pham, A. T., Malterud, K. E., Paulsen, B. S., Diallo, D., and Wangensteen, H. (2014). alpha-glucosidase inhibition, 15-lipoxygenase inhibition, and brine shrimp toxicity of extracts and isolated compounds from Terminalia macroptera leaves. Pharm. Biol. 52, 1166-1169. doi: 10.3109/13880209.2014.880486

Pinheiro Silva, L., Damacena De Angelis, C., Bonamin, F., Kushima, H., José Mininel, F., Campaner Dos Santos, L., et al. (2015). Terminalia catappa L.: a medicinal plant from the Caribbean pharmacopeia with anti Helicobacter pylori and antiulcer action in experimental rodent models. J. Ethnopharmacol. 15, 285-295. doi: 10.1016/j.jep.2014.11.025

Priya, N., Mathur, K., Sharma, A., Agrawal, R., Agarwal, V., and Acharya, J. (2019). Effect of Terminalia Arjuna on total platelet count and lipid profile in patients of coronary artery disease. Adv. Hum. Biol. 9, 98-101. doi: 10.4103/AIHB.AIHB_8_18

Rahimi, V. B., Askari, V. R., Shirazinia, R., Soheili-Far, S., Askari, N., RahmanianDevin, P., et al. (2018). Protective effects of hydro-ethanolic extract of Terminalia chebula on primary microglia cells and their polarization (M1/M2 balance). Multiple Sclerosis Rel. Disord. 25, 5-13. doi: 10.1016/j.msard.2018.07.015

Rashed, K., Potočnjak, I., Giacometti, J., Škoda, M., and Domitrović, R. (2014). Terminalia bellerica aerial parts ethyl acetate extract exhibits antioxidant, antiinflammatory and antifibrotic activity in carbon tetrachloride-intoxicated mice. J. Funct. Foods 8, 319-330. doi: 10.1016/j.jff.2014.03.033

Rayan, P., Matthews, B., Mcdonnell, P. A., and Cock, I. E. (2015). Terminalia ferdinandiana extracts as inhibitors of Giardia duodenalis proliferation: a new treatment for giardiasis. Parasitol. Res. 114, 2611-2620. doi: 10.1007/s00436015-4465-4

Reddy, T. C., Aparoy, P., Babu, N. K., Kumar, K. A., Kalangi, S. K., and Reddanna, P. (2010). Kinetics and docking studies of a COX-2 inhibitor isolated from Terminalia bellerica fruits. Protein Pept. Lett. 17, 1251-1257. doi: 10.2174/092986610792231537

Reddy, M. M., Devavarann, J. D., Dhas, J., Adeghate, E., and Ennerald, B. S. (2015). Antihyperlipidemic effect of methanol bark extract of Terminalia chebula in male albino Wistar rats. Pharm. Biol. 53, 1133-1140. doi: 10.3109/13880209.2014.962058

Rohaeti, E., and Rakhmawati, A. (2017). Application of Terminalia Catappa in preparation of silver nanoparticles to develop antibacterial nylon. Oriental J. Chem. 33, 2905-2912. doi: 10.13005/ojc/330625

Ruf, J. (1999). Wine and polyphenols related to platelet aggregation and atherothrombosis. Drugs Under Exp. Clin. Res. 25, 125-131.

Sadeghnia, H. R., Jamshidi, R., Afshari, A. R., Mollazadeh, H., Forouzanfar, F., and Rakhshandeh, H. (2017). Terminalia chebula attenuates quinolinate-induced oxidative PC12 and OLN-93 cell death. Multiple Sclerosis Rel. Disord. 14, 6067. doi: 10.1016/j.msard.2017.03.012

Saha, S., and Verma, R. J. (2016). Antioxidant activity of polyphenolic extract of Terminalia chebula Retzius fruits. J. Taibah Univ. Sci. 10, 805-812. doi: 10.1016/j.jtusci.2014.09.003

Saivaraj, S., and Chandramohan, G. (2018). Antimicrobial activity of natural dyes obtained from Terminalia arjuna (Roxb.) Wight \& Arn barks. World Sci. News 98, 221-227.

Saleem, A., Husheem, M., Härkönen, P., and Pihlaja, K. (2002). Inhibition of cancer cell growth by crude extract and the phenolics of Terminalia chebula retz. fruit. J. Ethnopharmacol. 81, 327-336. doi: 10.1016/S0378-8741(02)00099-5 
Salih, E. Y., Fyhrquist, P., Abdalla, A., Abdelgadir, A. Y., Kanninen, M., Sipi, M., et al. (2017). LC-MS/MS tandem mass spectrometry for analysis of phenolic compounds and pentacyclic triterpenes in antifungal extracts of Terminalia brownii (Fresen). Antibiotics 6, 37. doi: 10.3390/antibiotics6040037

Salih, E. Y. A., Julkunen-Tiitto, R., Lampi, A.-M., Kanninen, M., Luukkanen, O., Sipi, M., et al. (2018). Terminalia laxiflora and Terminalia brownii contain a broad spectrum of antimycobacterial compounds including ellagitannins, ellagic acid derivatives, triterpenes, fatty acids and fatty alcohols. J. Ethnopharmacol. 227, 82-96. doi: 10.1016/j.jep.2018.04.030

Shalom, J., and Cock, I. E. (2018). Terminalia ferdinandiana Exell. fruit and leaf extracts inhibit proliferation and induce apoptosis in selected human cancer cell lines. Nutr. Cancer 70, 579-593. doi: 10.1080/01635581.2018.1460680

Sharma, P., Shoeb, A., Kapil, R., and Popli, S. (1982). "Arjunolone-a new flavone from stem bark of Terminalia arjuna. Indian J. Chem. Including Med. Chem. 21, 263-264.

Sharma, C., Aneja, K. R., Kasera, R., and Aneja, A. J. W. J. O. sO. (2012). Antimicrobial potential of Terminalia chebula Retz. fruit extracts against ear pathogens. World J. Otorhinolaryngol. 2, 8-13. doi: 10.5319/wjo.v2.i2.8

Shen, Y.-C., Juan, C.-W., Lin, C.-S., Chen, C.-C., and Chang, C.-L. (2017). Neuroprotective effect of Terminalia chebula extracts and ellagic acid in pc12 cells. Afr. J. Tradit. Complement. Altern. Medicines 14, 22-30. doi: 10.21010/ajtcam.v14i4.3

Sheng, Z., Zhao, J., Muhammad, I., and Zhang, Y. (2018). Optimization of total phenolic content from Terminalia chebula Retz. fruits using response surface methodology and evaluation of their antioxidant activities. PloS One 13, e0202368-e0202368. doi: 10.1371/journal.pone.0202368

Shyni, G. L., Kavitha, S., Indu, S., Arya, A. D., Anusree, S. S., Vineetha, V. P., et al. (2014). Chebulagic acid from Terminalia chebula enhances insulin mediated glucose uptake in 3T3-L1 adipocytes via PPAR $\gamma$ signaling pathway. BioFactors 40, 646-657. doi: 10.1002/biof.1193

Silva, O., Duarte, A., Cabrita, J., Pimentel, M., Diniz, A., and Gomes, E. (1996). Antimicrobial activity of Guinea-Bissau traditional remedies. J. Ethnopharmacol. 50, 55-59. doi: 10.1016/0378-8741(95)01323-7

Sivamaruthi, B. S., Ramkumar, V. S., Archunan, G., Chaiyasut, C., and Suganthy, N. (2019). Biogenic synthesis of silver palladium bimetallic nanoparticles from fruit extract of Terminalia chebula - In vitro evaluation of anticancer and antimicrobial activity. J. Drug Delivery Sci. Technol. 51, 139-151. doi: 10.1016/j.jddst.2019.02.024

Somarathna, T., Ranaweera, K. K. D. S., Premakumara, G., and Weerakkody, N. S. (2018). Combination effect of Alpinia malaccensis and Terminalia catappa extract for controlling foodborne pathogens and spoilage bacteria on ready to cook vacuum packed raw chicken meat. J. Food Saf. 38, e12562. doi: 10.1111/ffs.12562

Suganthy, N., Muniasamy, S., and Archunan, G. (2018). Safety assessment of methanolic extract of Terminalia chebula fruit, Terminalia arjuna bark and its bioactive constituent 7-methyl gallic acid: In vitro and in vivo studies. Regul. Toxicol. Pharmacol. 92, 347-357. doi: 10.1016/j.yrtph.2017.12.019

Suparno, O., Panandita, T., Afifah, A., and Purnawati, R. (2018). Conference Series: Earth and Environmental Science Vol. 141 (IOP Publishing), 012028.

Tabuti, J. R., Lye, K. A., and Dhillion, S. S. (2003). Traditional herbal drugs of Bulamogi, Uganda: plants, use and administration. J. Ethnopharmacol. 88, 1944. doi: 10.1016/S0378-8741(03)00161-2

Tawfike, A. F., Abbott, G., Young, L., and Edrada-Ebel, R. (2018). Metabolomic-guided isolation of bioactive natural products from Curvularia sp., an endophytic fungus of Terminalia laxiflora. Planta Med. 84, 182-190. doi: 10.1055/s-0043-118807

Terças, A. G., Monteiro, A. D. S., Moffa, E. B., Dos Santos, J. R. A., De Sousa, E. M., Pinto, A. R. B., et al. (2017). Phytochemical Characterization of Terminalia catappa Linn. extracts and their antifungal activities against Candida spp. Front. Microbiol. 8, 595-595. doi: 10.3389/fmicb.2017.00595

Toppo, E., Sylvester Darvin, S., Esakkimuthu, S., Buvanesvaragurunathan, K., Ajeesh Krishna, T. P., Antony Caesar, S., et al. (2018). Curative effect of arjunolic acid from Terminalia arjuna in non-alcoholic fatty liver disease models. Biomed. Pharmacother. 107, 979-988. doi: 10.1016/j.biopha.2018.08.019

Upadhyay, B., Singh, K. P., and Kumar, A. (2011). Ethno-veterinary uses and informants consensus factor of medicinal plants of Sariska region, Rajasthan, India. J. Ethnopharmacol. 133, 14-25. doi: 10.1016/j.jep.2010.08.054
Vemuri, P. K., Dronavalli, L., Nayakudugari, P., Kunta, A., and Challagulla, R. (2019). Phytochemical Analysis and biochemical characterization of Terminalia Chebula extracts for its medicinal use. Biomed. Pharmacol. J. 12, 1525-1529. doi: 10.13005/bpj/1783

Vidya, A. G., Vijayan, A., Jyothis, L., Nair, R., and Suja, K. (2019). Evaluation of antifungal efficacy of some medicinal plants on Candida spp. causing vulvovaginitis. Indian J. Exp. Biol. 57, 297-301.

Wang, M., Yang, L., Ji, M., Zhao, P., Sun, P., Bai, R., et al. (2015). Aqueous extract of Terminalia chebula induces apoptosis in lung cancer cells via a mechanism involving mitochondria-mediated pathways. Braz. Arch. Biol. Technol. 58, 208-215. doi: 10.1590/S1516-8913201400202

World Health Organization (1999). WHO monographs on selected medicinal plants (Vol. 2): World Health Organization (World Health Organization).

World Health Organization (2018). Global Health Observatory Data (World Health Organization). Available at: http://www.who.int/gho/en/.

World Health Organization (2019). Cancer (World Health Organization). Available at: https://www.who.int/health-topics/cancer\#tab=tab_1.

Wright, M. H., Sirdaarta, J., White, A., Greene, A. C., and Cock, I. E. (2016). Bacillus anthracis growth inhibitory properties of Australian Terminalia spp.: Putative identification of low polarity volatile components by GC-MS headspace analysis. Pharmacognosy J. 8, 281-289. doi: 10.5530/pj.2016.3.18

Wright, M. H., Shalom, J., Matthews, B., Greene, A. C., and Cock, I. E. (2019). Terminalia ferdinandiana Exell: extracts inhibit Shewanella spp. growth and prevent fish spoilage. Food Microbiol. 78, 114-122. doi: 10.1016/j.fm.2018. 10.006

Yallappa, S., Manjanna, J., Sindhe, M. A., Satyanarayan, N. D., Pramod, S. N., and Nagaraja, K. (2013). Microwave assisted rapid synthesis and biological evaluation of stable copper nanoparticles using T. arjuna bark extract. Spectrochim. Acta Part A: Mol. Biomol. Spectrosc. 110, 108-115. doi: 10.1016/ j.saa.2013.03.005

Yang, M. H., Vasquez, Y., Ali, Z., Khan, I. A., and Khan, S. I. (2013). Constituents from Terminalia species increase PPAR alpha and PPAR gamma levels and stimulate glucose uptake without enhancing adipocyte differentiation. J. Ethnopharmacol. 149, 490-498. doi: 10.1016/j.jep.2013.07.003

Yeh, C.-B., Yu, Y.-L., Lin, C.-W., Chiou, H.-L., Hsieh, M.-J., and Yang, S.-F. (2014). Terminalia catappa attenuates urokinase-type plasminogen activator expression through Erk pathways in Hepatocellular carcinoma. BMC Complement. Altern. Med. 14, 141. doi: 10.1186/1472-6882-14-141

Zhang, X., He, L., Lu, Q., and Li, D. (2016). Pharmacological activity of Terminalia chebula. Zhongguo Zhong yao za zhi= Zhongguo zhongyao zazhi= China J. Chin. Materia Med. 41, 619-623. doi: 10.4268/cjcmm20160412

Zhang, X.-R., Kaunda, J. S., Zhu, H.-T., Wang, D., Yang, C.-R., and Zhang, Y.-J. (2019). The genus Terminalia (Combretaceae): An ethnopharmacological, phytochemical and pharmacological review. Natural Prod. Bioprospect. 9, 357-392. doi: 10.1007/s13659-019-00222-3

Zhong, L., Bornman, J. F., Wu, G., Hornoff, A., Dovi, K., Al-Ali, H., et al. (2018). The nutritional and phytochemical composition of the indigenous Australian pindan walnut (Terminalia cunninghamii) Kernels. Plant Foods Hum. Nutr. 73, 40-46. doi: 10.1007/s11130-017-0647-9

Conflict of Interest: The authors declare that the research was conducted in the absence of any commercial or financial relationships that could be construed as a potential conflict of interest.

Copyright (c) 2020 Das, Kim, Fan, Gutiérrez-Grijalva, Heredia, Nissapatorn, Mitsuwan, Pereira, Nawaz, Siyadatpanah, Norouzi, Sawicka, Shin and Patra. This is an open-access article distributed under the terms of the Creative Commons Attribution License (CC BY). The use, distribution or reproduction in other forums is permitted, provided the original author(s) and the copyright owner(s) are credited and that the original publication in this journal is cited, in accordance with accepted academic practice. No use, distribution or reproduction is permitted which does not comply with these terms. 